Aus der Klinik für Hämatologie und Onkologie

(Prof. Dr. med. L. Trümper)

der Medizinischen Fakultät der Universität Göttingen

\title{
Prognostische Bedeutung seltener \\ Einzelchromosomenanomalien bei \\ myelodysplastischen Syndromen
}

\author{
INAUGURAL-DISSERTATION \\ zur Erlangung des Doktorgrades \\ der Medizinischen Fakultät der \\ Georg-August-Universität zu Göttingen
}

vorgelegt von

Bärbel Christa Elfriede Rothmann

aus

Kassel

Göttingen 2019 
Dekan:

Referent/in

Ko-Referent/in:

Drittreferent/in:
Prof. Dr. rer. nat. H. K. Kroemer

PD Dr. Julie Schanz

Prof. Dr. Heidi Eva Hahn

Prof. Dr. Margarete Schön

Datum der mündlichen Prüfung: 15.01.2020 
Hiermit erkläre ich, die Dissertation mit dem Titel "Prognostische Bedeutung seltener Einzelchromosomenanomalien bei myelodysplastischen Syndromen" eigenständig angefertigt und keine anderen als die von mir angegebenen Quellen und Hilfsmittel verwendet zu haben.

Göttingen, den 


\section{Inhaltsverzeichnis}

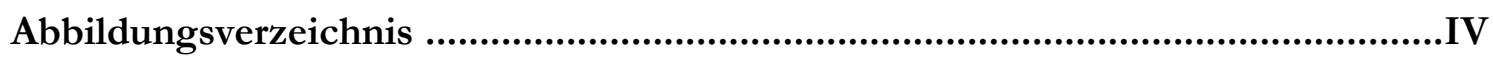

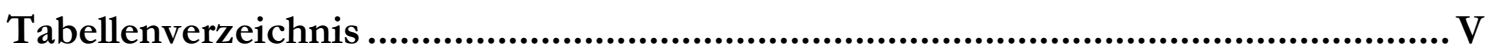

Abkürzungsverzeichnis.........................................................................................

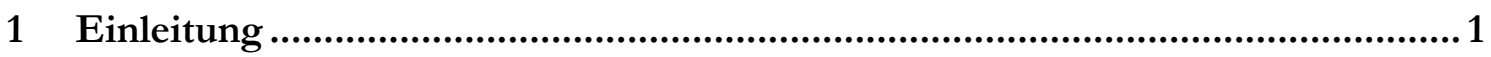

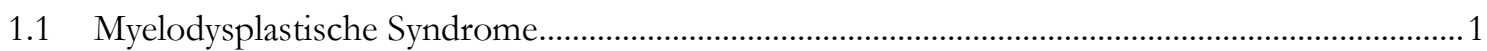

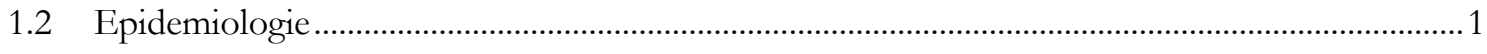

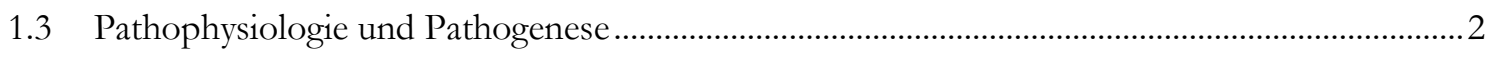

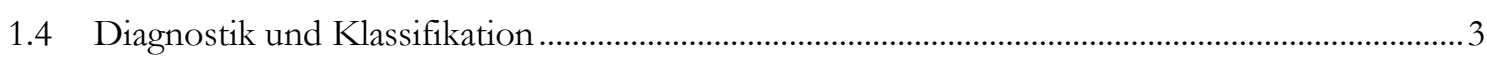

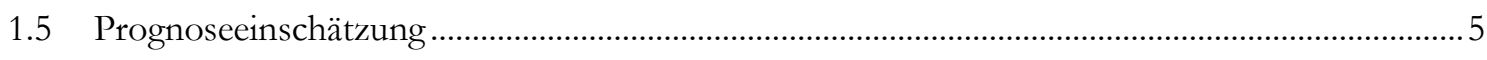

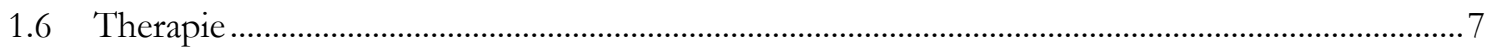

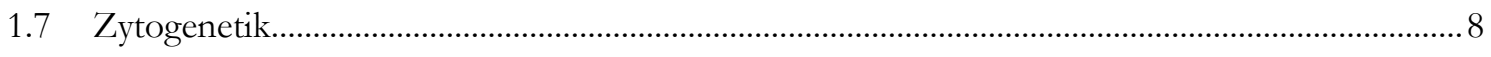

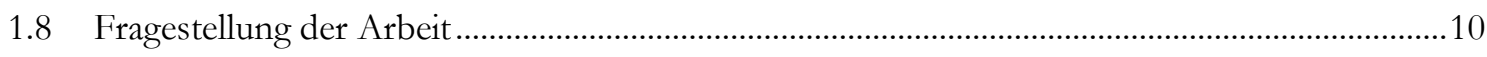

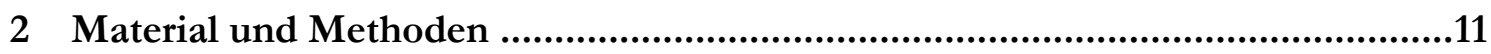

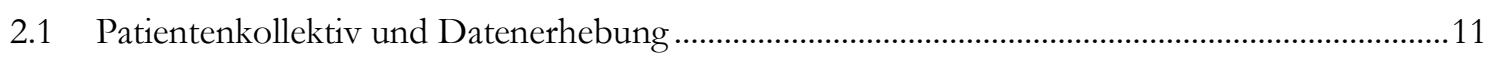

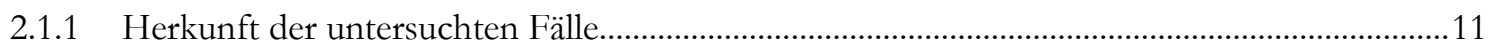

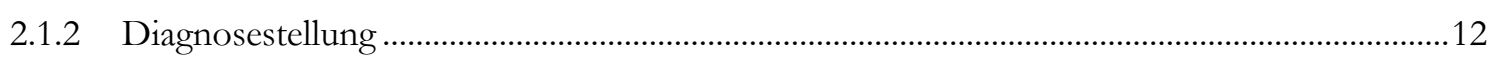

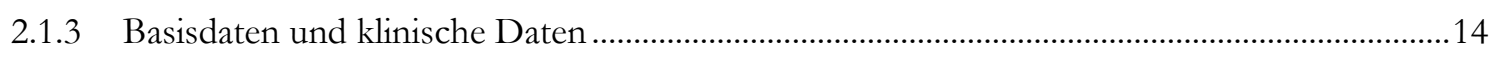

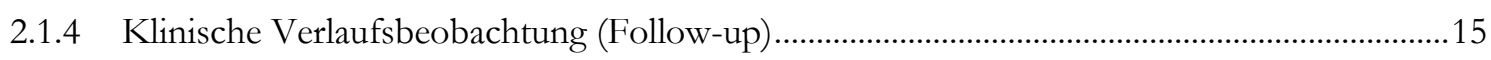

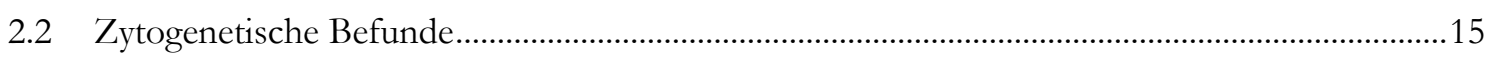

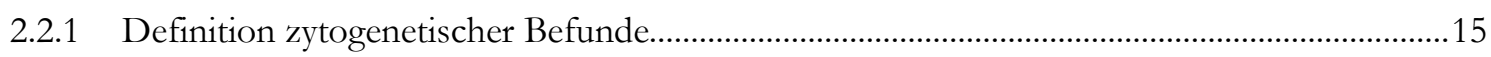

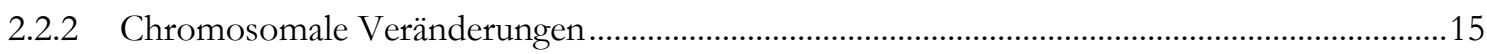

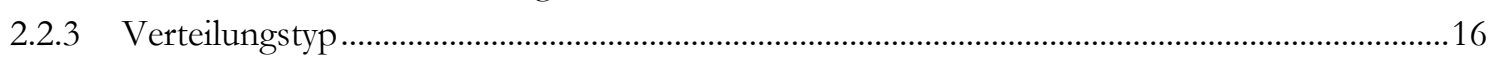

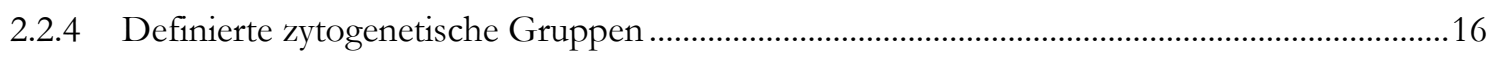

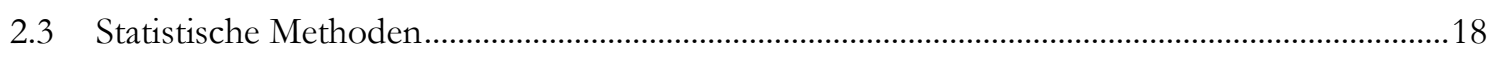

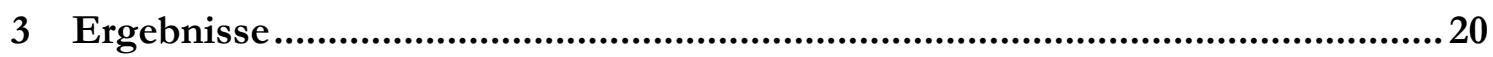

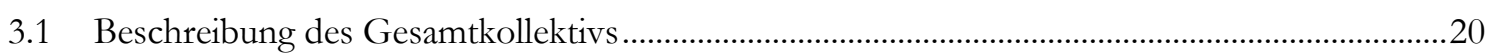

3.1.1 Häufigkeiten der Einzelchromosomenanomalien im Gesamtkollektiv......................................20

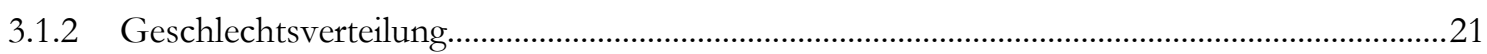

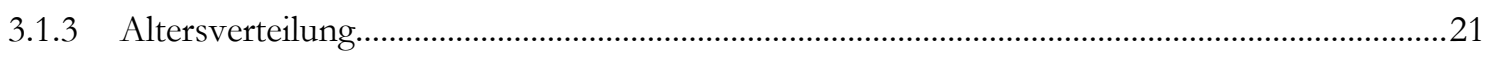

3.1.4 MDS-Subtypen nach FAB-Klassifikation im Gesamtkollektiv ................................................22

3.1.5 MDS-Subtypen nach WHO-Klassifikation 2001 und $2008 \mathrm{im}$ Gesamtkollektiv .....................22

3.1.6 Überlebenszeit (ÜLZ) für das Gesamtkollektiv ....................................................................23

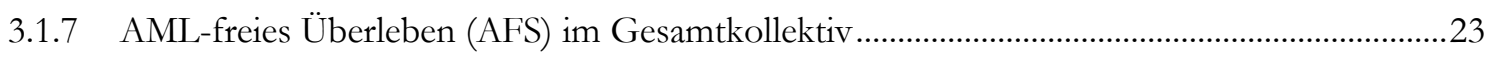

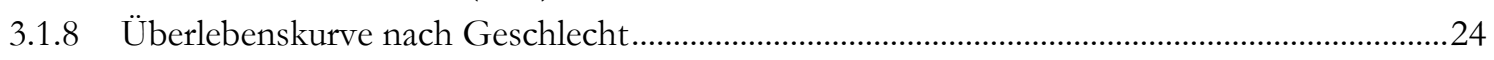


3.2 Univariate Analyse nach zytogenetischen Subgruppen ...............................................................24

3.2.1 Überlebenszeit in der Subgruppe von Patienten mit Trisomie (1q) (TR1Q) ...........................25

3.2.2 AML-freie Überlebenszeit in der Subgruppe von Patienten mit Trisomie (1q) (TR1Q) .....25

3.2.3 Überlebenszeit in der Subgruppe von Patienten mit Monosomie 3 (MO3) ............................26

3.2.4 AML-freie Überlebenszeit in der Subgruppe von Patienten mit Monosomie 3 (MO3) ........26

3.2.5 Überlebenszeit in der Subgruppe von Patienten mit Monosomie 5 (MO5) .............................27

3.2.6 AML-freie Überlebenszeit in der Subgruppe von Patienten mit Monosomie 5 (MO5) ........27

3.2.7 Überlebenszeit in der Subgruppe von Patienten mit Veränderungen am Chromosom 6 (ANY6) ..... .28

3.2.8 AML-freie Überlebenszeit in der Subgruppe von Patienten mit Veränderungen am

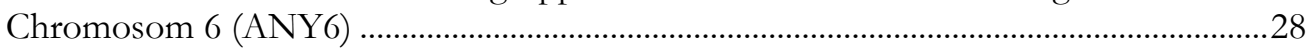

3.2.9 Überlebenszeit in der Subgruppe von Patienten mit Monosomie 9 (MO9) .............................29

3.2.10 AML-freie Überlebenszeit in der Subgruppe von Patienten mit Monosomie 9 (MO9) ........29

3.2.11 Überlebenszeit in der Subgruppe von Patienten mit Veränderungen am Chromosom

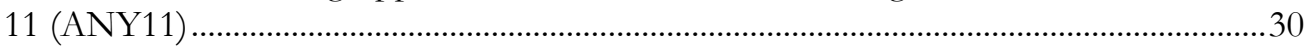

3.2.12 AML-freie Überlebenszeit in der Subgruppe von Patienten mit Veränderungen am Chromosom 11 (ANY11) .................................................................................................

3.2.13 Überlebenszeit in der Subgruppe von Patienten mit Monosomie 12 (MO12) ........................31

3.2.14 AML-freie Überlebenszeit in der Subgruppe von Patienten mit Monosomie 12

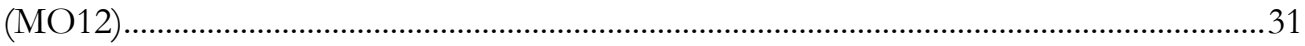

3.2.15 Überlebenszeit in der Subgruppe von Patienten mit Monosomie (13q) (MO13Q) ................32

3.2.16 AML-freie Überlebenszeit in der Subgruppe von Patienten mit Monosomie (13q)

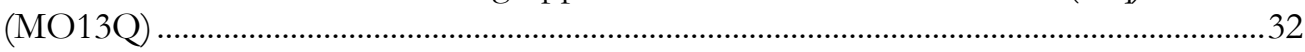

3.2.17 Überlebenszeit in der Subgruppe von Patienten mit Monosomie 14 (MO14) ........................33

3.2.18 AML-freie Überlebenszeit in der Subgruppe von Patienten mit Monosomie 14 (MO14).

3.2.19 Überlebenszeit in der Subgruppe von Patienten mit Monosomie 16 (MO16)

3.2.20 AML-freie Überlebenszeit in der Subgruppe von Patienten mit Monosomie 16

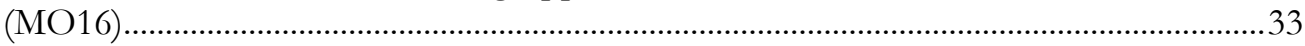

3.2.21 Überlebenszeit in der Subgruppe von Patienten mit Monosomie 18 (MO18) ........................34

3.2.22 AML-freie Überlebenszeit in der Subgruppe von Patienten mit Monosomie 18 (MO18)

3.2.23 Überlebenszeit in der Subgruppe von Patienten mit Veränderungen am Chromosom 20 (ANY20)

3.2.24 AML-freie Überlebenszeit in der Subgruppe von Patienten mit Veränderungen am

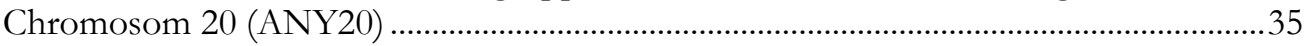

3.2.25 Überlebenszeit in der Subgruppe von Patienten mit Trisomie 21 (TR21)...............................36

3.2.26 AML-freie Überlebenszeit in der Subgruppe von Patienten mit Trisomie 21 (TR21)...........36

3.2.27 Überlebenszeit in der Subgruppe von Patienten mit Monosomie X (MOX)...........................37

3.2.28 AML-freie Überlebenszeit in der Subgruppe von Patienten mit Monosomie X (MOX)......37

3.2.29 Überlebenszeit in der Subgruppe von Patienten mit derivativem Chromosom $(1 ; 7)$ $\operatorname{DER}(1 ; 7)$

3.2.30 AML-freie Überlebenszeit in der Subgruppe von Patienten mit derivativem Chromosom (1;7) (DER1;7)

3.2.31 Überlebenszeit in der Subgruppe von Patienten mit balancierten Translokationen (TRB).

3.2.32 AML-freie Überlebenszeit in der Subgruppe von Patienten mit balancierten Translokationen (TRB)

3.2.33 Überlebenszeit in der Subgruppe der Patienten mit Markerchromosom (MAR) 
3.2.34 AML-freie Überlebenszeit in der Subgruppe der Patienten mit Markerchromosom (MAR)

3.2.35 Überlebenszeit in der Subgruppe von Patienten mit allen anderen

Einzelchromosomenanomalien (OTH)

3.2.36 AML-freie Überlebenszeit in der Subgruppe von Patienten mit allen anderen Einzelchromosomenanomalien (OTH)...

3.3 Multivariate Überlebensanalyse der Subgruppen ......................................................................42

3.3.1 Multivariate Analyse der Überlebenszeit ...................................................................................42

3.3.2 Multivariate Analyse der AML-freien Überlebenszeit..............................................................44

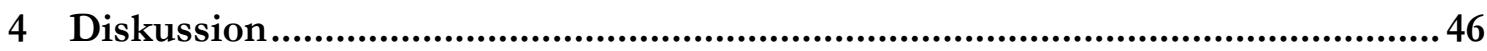

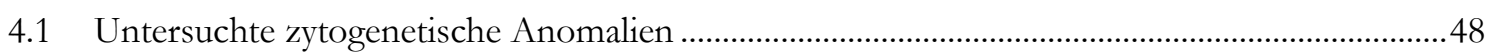

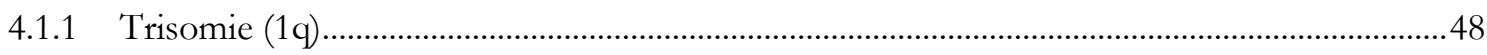

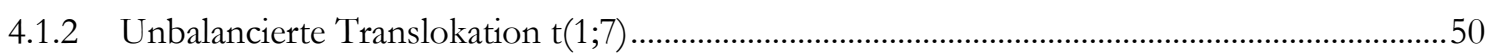

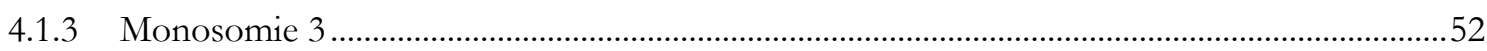

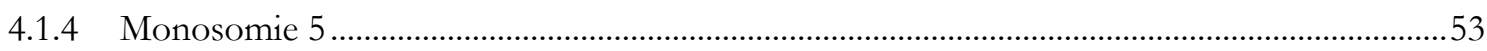

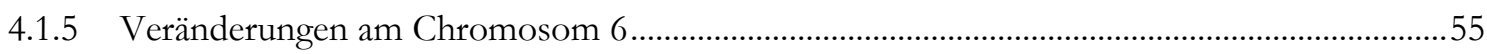

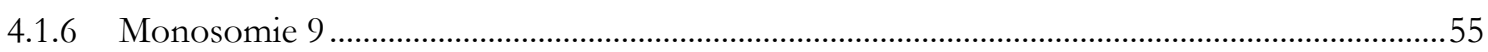

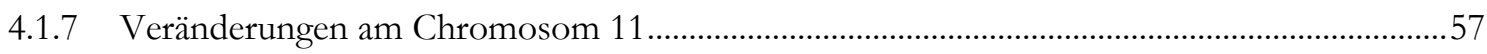

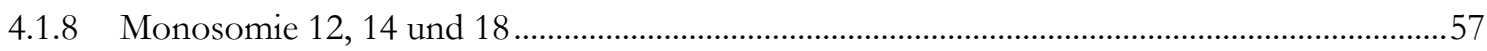

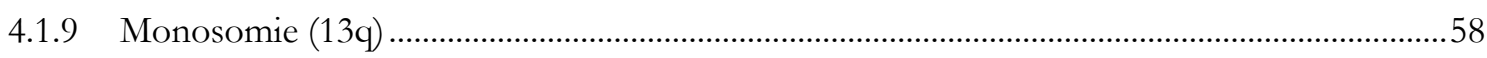

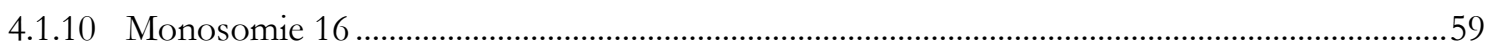

4.1.11 Veränderungen am Chromosom 20 …............................................................................60

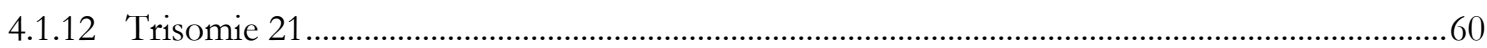

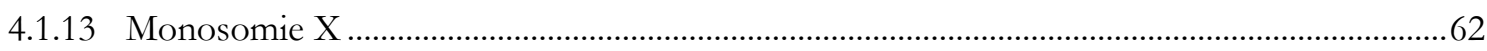

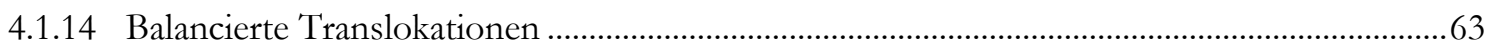

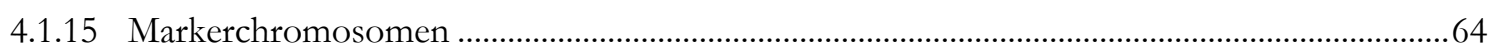

4.1.16 Einzelchromosomaberrationen mit einer absoluten Häufigkeit von < 5 Fällen....................65

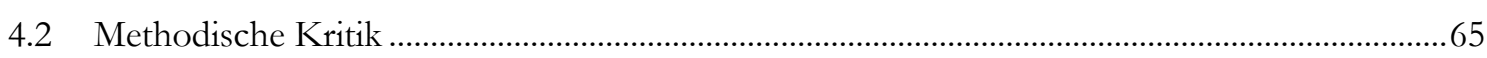

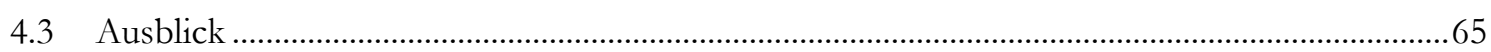

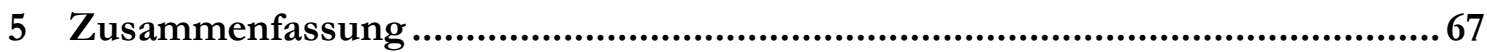

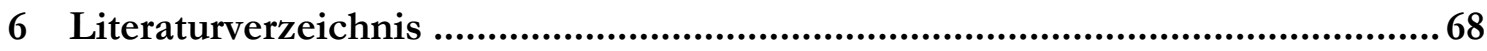




\section{Abbildungsverzeichnis}

Abbildung 1: Geschlechtsverteilung im Gesamtkollektiv ......................................................................21

Abbildung 2: Prozentuale Anteile am Gesamtkollektiv nach FAB-Klassifikation .................................22

Abbildung 3: Prozentuale Anteile am Gesamtkollektiv nach WHO-Klassifikation...............................22

Abbildung 4: Kaplan-Meier-Überlebensfunktion für alle Patienten ......................................................23

Abbildung 5: Kaplan-Meier-Überlebensfunktion nach Geschlecht....................................................24

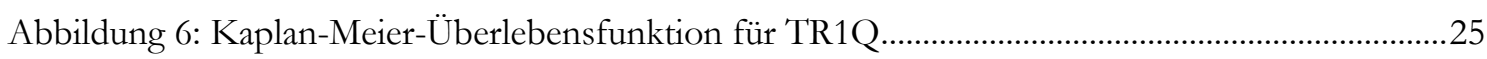

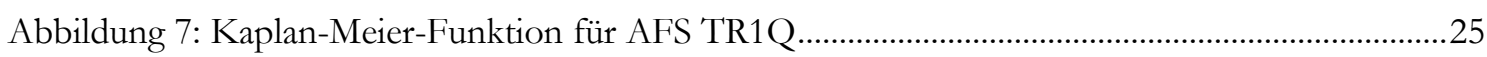

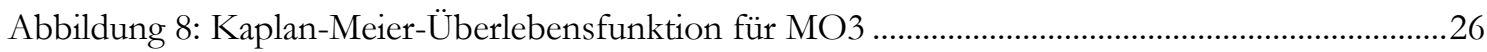

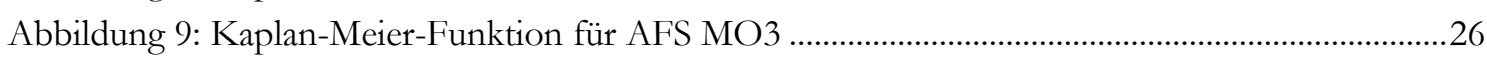

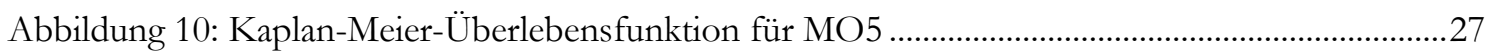

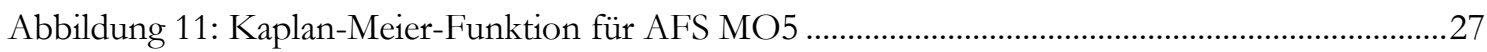

Abbildung 12: Kaplan-Meier-Überlebensfunktion für ANY6 ..............................................................2

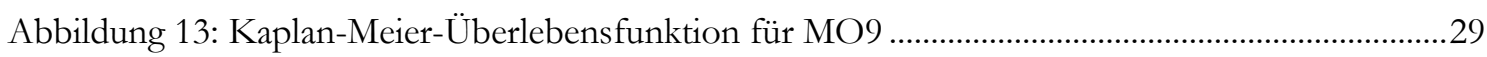

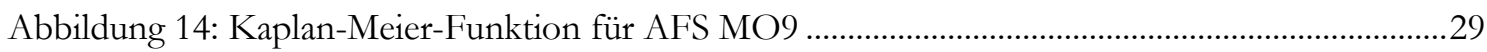

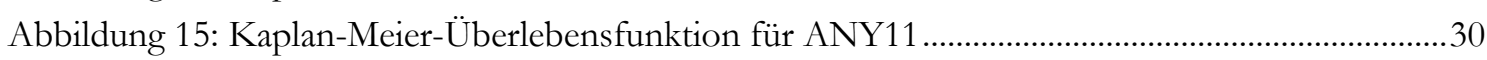

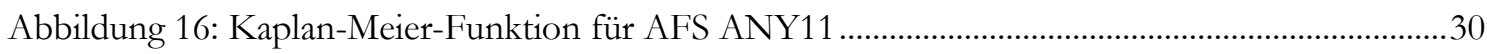

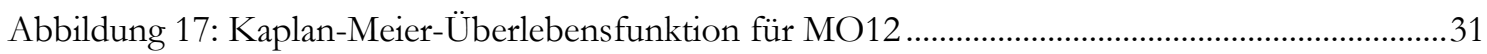

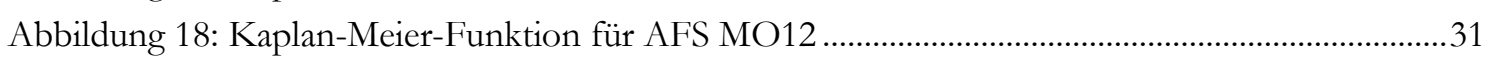

Abbildung 19: Kaplan-Meier-Überlebensfunktion für MO13Q...............................................................

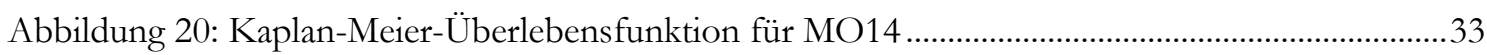

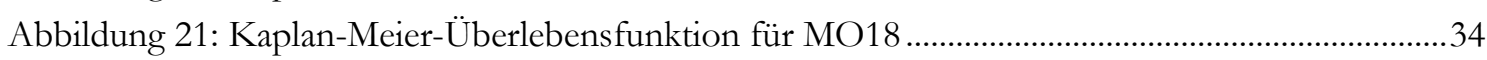

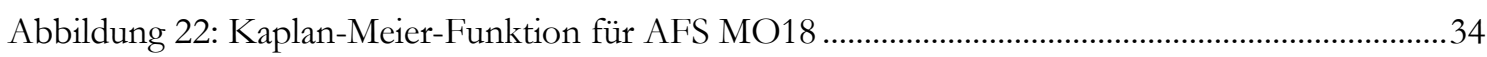

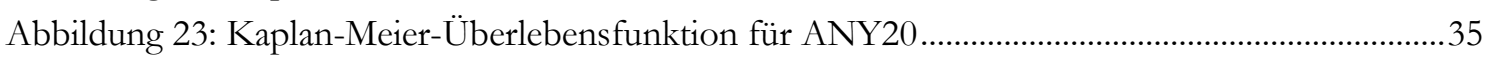

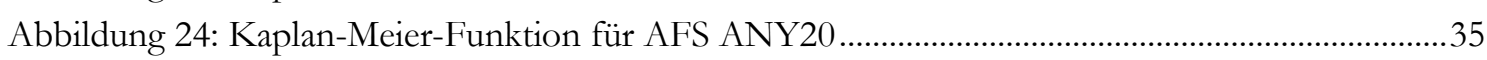

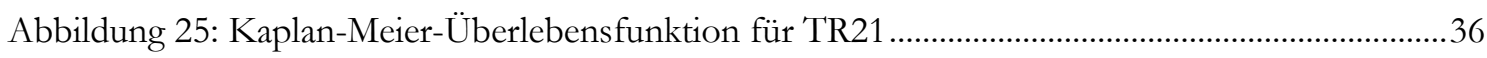

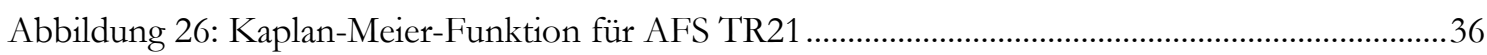

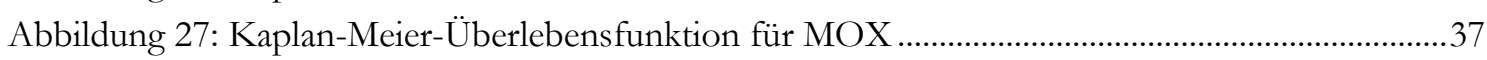

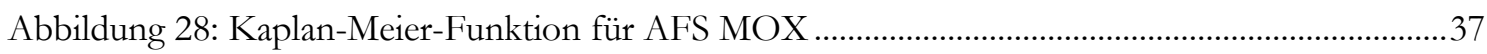

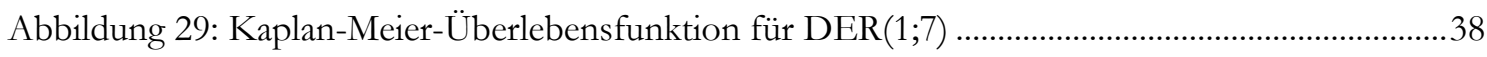

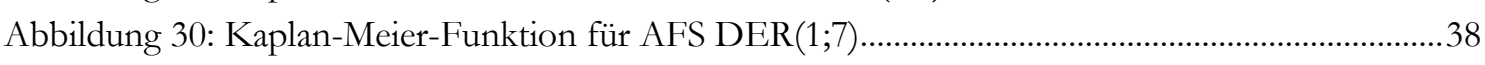

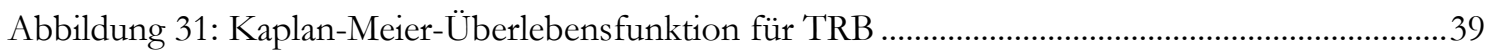

Abbildung 32: Kaplan-Meier-Funktion für AFS TRB ........................................................................

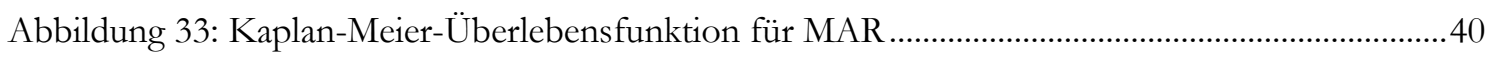

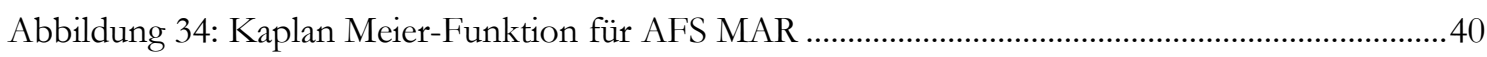

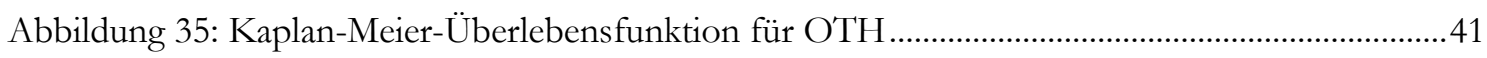

Abbildung 36: Kaplan-Meier-Funktion für AFS OTH ..................................................................... 41 


\section{Tabellenverzeichnis}

Tabelle 1: WHO-Klassifikation Myelodysplastischer Syndrome nach Arber..........................................

Tabelle 2: International Prognostic Scoring System (IPSS) nach Greenberg ………….........................5

Tabelle 3: Revised International Prognostic Scoring System (IPSS-R) nach Greenberg.........................6

Tabelle 4: WHO-adaptiertes Prognostic Scoring System (WPSS) nach Malcovati..................................

Tabelle 5: FAB-Klassifikation Myelodysplastischer Syndrome nach Bennett......................................12

Tabelle 6: WHO-Klassifikation Myelodysplastischer Syndrome nach Vardiman..................................13

Tabelle 7: Absolute und relative Häufigkeitsverteilung der untersuchten Aberrationen.......................20

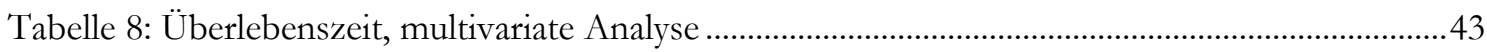

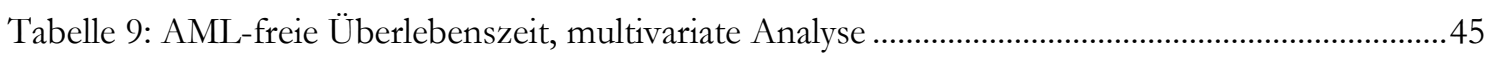




\section{Abkürzungsverzeichnis}

AFS

AML

ANC

CMML

del

FAB

FISH

$\mathrm{Hb}$

HR

IPSS

IPSS-R

ISCN

KM

KI

MDS

MDS-U

MPN

NOR

$\mathrm{p}$

$\mathrm{pB}$

PLT

q

RA

RARS

$\operatorname{RAEB}(-\mathrm{T})$

RCUD/RCMD

ÜLZ

WHO

WPSS
AML-freie Überlebenszeit (AML-free survival)

akute myeloische Leukämie

absolute Neutrophilenzahl (absolute neutrophil count)

chronische myelomonozytäre Leukämie

Deletion

French-American-British Co-operative Group

Fluoreszenz-in-situ-Hybridisierung

Hämoglobin

Hazard Ratio

International Prognostic Scoring System

Revised International Prognostic Scoring System

International System for Human Cytogenetic Nomenclature

Knochenmark

Konfidenzintervall

myelodysplastische Syndrome

unklassifizierbares MDS

myeloproliferative Neoplasie

Fälle mit normalem Karyotyp

kurzer Arm eines Chromosoms

peripheres Blut

Thrombozyten (platelets)

langer Arm eines Chromosoms

refraktäre Anämie

refraktäre Anämie mit Ringsideroblasten

refraktäre Anämie mit Excess von Blasten (-in Transformation)

refraktäre Zytopenie mit uni-/multilineärer Dysplasie

Überlebenszeit

World Health Organization

WHO-adaptiertes Prognostic Scoring System 


\section{$1 \quad$ Einleitung}

\subsection{Myelodysplastische Syndrome}

Die Bezeichnung Myelodysplastische Syndrome (MDS) stellt einen Sammelbegriff dar. Dahinter verbirgt sich eine heterogene Gruppe erworbener klonaler Stammzellerkrankungen des blutbildenden Systems, die durch fortschreitende Zytopenien im peripheren Blut, bei gleichzeitig hyperzellulärem Knochenmark (Fisher et al. 1973), und durch ein erhöhtes Risiko der Entwicklung einer akuten myeloischen Leukämie gekennzeichnet sind (Greenberg 1983; Tricot 1992). Klinisch fallen die Patienten häufig mit den Symptomen einer Anämie, wie Blässe von Haut und Schleimhäuten, Leistungsminderung und Müdigkeit, auf; bei hochgradiger Anämie können auch Belastungs- und Ruhedyspnoe auftreten. Infolge einer Granulozytopenie kann eine Infektneigung bestehen. Blutungsstigmata treten mitunter als Ausdruck einer Thrombozytopenie auf, und jede Form der Zytopenie kann zu einer verkürzten Überlebenszeit der Patienten beitragen (Weisdorf et al. 1983; Doll und List 1989). Generell zeigen ca. ein Drittel der Patienten einen stabilen Krankheitsverlauf, ein Drittel erleidet lebenslimitierende Komplikationen infolge der Zytopenien und ein Drittel zeigt einen Progress in eine akute myeloische Leukämie (Tricot et al. 1985). In der Regel sind ältere Menschen betroffen, die Prognose variiert stark zwischen wenigen Monaten bis zu mehreren Jahren, und die Intensität der Behandlung orientiert sich u. a. an der zu erwartenden individuellen Prognose (Tricot 1992). Bei den häufig älteren Patienten sind darüber hinaus eventuelle Komorbiditäten, die ein erhöhtes Risiko für die Therapieverträglichkeit darstellen können, zu berücksichtigen (Greenberg et al. 2017).

Neben myelodysplastischen Syndromen als de-novo-Erkrankungen ungeklärter Ätiologie kommen als therapieassoziierte Erkrankungen auch sekundäre MDS nach Vorbehandlung mit ionisierender Strahlung oder Zytostatika (v. a. Alkylanzien) vor. Diese sekundären MDS haben eine besonders schlechte Prognose (Kantajian und Keating 1987).

\subsection{Epidemiologie}

Die Inzidenz der myelodysplastischen Syndrome beträgt 4-5/100 000 und steigt mit dem Alter an. Bei Patienten, die älter als 70 Jahre sind, beträgt die Inzidenz ca. 23-50/100 000. Dabei sind Männer häufiger betroffen als Frauen (Aul et al. 1992). Damit handelt es sich bei den myelodysplastischen Syndromen um vergleichsweise häufige Knochenmarkstammzellerkrankungen, vor allem im fortgeschrittenen Lebensalter, denn nur ca. 6\% der Patienten sind jünger als 50 Jahre und das mediane Erkrankungsalter liegt bei 72 Jahren (Germing et al. 2004). 


\subsection{Pathophysiologie und Pathogenese}

Die Pathogenese der myelodysplastischen Syndrome ist seit mehreren Jahrzehnten Gegenstand intensiver Forschung. Trotzdem sind viele Mechanismen in der komplexen und vielschichtigen Entwicklung bis zur klinischen Präsentation mit den Zeichen einer ineffektiven Hämatopoese bis heute nicht verstanden. Dass myelodysplastische Syndrome mit einer malignen Transformation der pluripotenten Stammzelle einhergehen, wurde bereits früh beschrieben (Tricot et al. 1986). Auch der Nachweis einer schrittweisen Krankheitsentstehung über mehrere genetische Schritte, vergleichbar anderen Tumorentitäten, wurde früh nachgewiesen (Raskind et al. 1984). Gemein ist allen myelodysplastischen Syndromen als Ergebnis der malignen Transformation der hämatopoetischen Stammzelle eine ineffektive Hämatopoese (Bejar et al. 2011). Allerdings variieren dabei die unterschiedlichen klinischen Erscheinungsbilder so stark, dass dies nur durch multiple Mechanismen, die individuell einen unterschiedlich starken Einfluss auf die Pathogenese ausüben, erklärt werden kann. Da im Verlauf der Erkrankung oftmals durch zusätzliche genetische Veränderungen weitere Mechanismen hinzutreten, wird auch das Fortschreiten der Erkrankung mit Zunahme der Blutbildveränderungen oder der Übergang in eine sekundäre AML erklärbar. (Bejar et al. 2011).

Bejar et al. (2011) beschreiben verschiedene Schritte, die mit der Entstehung und Entwicklung myelodysplastischer Syndrome assoziiert sind: Zum einen ist hier die gesteigerte Selbsterneuerung der betroffenen Stammzelle und/oder die Entwicklung der Fähigkeit der Selbsterneuerung der Progenitorzellen zu nennen. Des weiteren spielt die gesteigerte Proliferationskapazität des malignen Stammzellklons oder der von ihm ausgehenden Progenitorzellen, sowie die beeinträchtigte oder blockierte Differenzierung eine wesentliche Rolle.

Die genetische und epigenetische Instabilität ist ein weiterer Schritt in der Krankheitsentwicklung und für den Krankheitsprogress. Hierdurch werden vor allem weitere Mutationen begünstigt, die dann das Krankheitsgeschehen vorantreiben. Wie bei anderen Tumoren scheinen auch bei den myelodysplastischen Syndromen die Umgehung der Kontrolle durch das angeborene Immunsystem, sowie anti-apoptotische Mechanismen im malignen Zellklon eine große Rolle zu spielen. Diese und weitere Mechanismen führen schließlich zu einer Unterdrückung der normalen Hämatopoese und gesteigerter, aber ineffektiven Hämatopoese und gesteigerter Apoptose mit dem Ergebnis unterschiedlich stark ausgeprägter Zytopenien im peripheren Blut (Bejar et al. 2011), während im Knochenmark normalerweise eine Hyperzellularität vorliegt. Außerdem spielen auch Dysregulation der Spiegel von Wachstumsfaktoren und Interleukinen im Knochenmarkstroma eine Rolle, durch die die normale Hämatopoese negativ beeinflusst wird (Bellamy 2001; Tsimberidou et al. 2008).

Zusammengefasst lassen sich zwar vielfältige chromosomale, genetische, epigenetische und biochemische Veränderungen bei Patienten mit MDS identifizieren und in den letzten Jahren 
auch zunehmend in ihren pathophysiologischen Auswirkungen erklären, vollständiges Verständnis liegt hier weiterhin noch nicht vor.

\subsection{Diagnostik und Klassifikation}

Der Verdacht auf das Vorliegen eines MDS ist angezeigt, wenn über mehr als 6 Monate unerklärte Zytopenien im peripheren Blut nachgewiesen werden können. Eine Zytopenie mindestens einer Zellreihe muss vorliegen und die Grenzen liegen hier bei einem Hämoglobin von $<10 \mathrm{~g} / \mathrm{dl}$, Thrombozyten $<100 \times 10^{9} / 1$ und $<1,8 \times 10^{9} / 1$ neutrophilen Granulozyten. Gleichzeitig dürfen die Monozyten einen Wert von 1x10 $/ 1$ nicht überschreiten (Zini 2017). Anamnestisch sollten sekundäre Ursachen einer Zytopenie, wie nephrologische oder hepatische Grunderkrankungen, und die vorausgegangenen Exposition gegenüber zytotoxischen Substanzen oder ionisierender Strahlung, sowie laborchemisch ein Vitamin B12- oder Folsäuremangel ausgeschlossen werden (Doll und List 1989). Im peripheren Blutausstrich lassen sich häufig bereits Dysplasiezeichen der roten Blutkörperchen und der neutrophilen Granulozyten nachweisen, was sich nach der Durchführung einer Knochenmarkspunktion mit Aspiration und Trepanation im Knochenmarksausstrich und der Knochenmarkshistologie bestätigt. Durch eine zusätzliche immunhistochemische Eisenfärbung kann auch der qualitative und quantitative Nachweis von Ringsideroblasten geführt werden (Zini 2017).

Zusätzlich gehört heute die Durchführung einer zytogenetischen Untersuchung mittels Chromosomenbänderungsanalyse und ggf. Fluoreszenz-in-situ-Hybrisdisierung (FISH) zur Standarddiagnostik - dies zum einen wegen der prognostischen Relevanz und zum anderen wegen inzwischen definierter typischer chromosomaler Aberrationen, die bei unerklärten Zytopenien ohne klassischen Befund in Blut und Knochenmark die Klonalität der Erkrankung belegen (Vardiman et al. 2009).

Mit der Diagnosestellung werden die myelodysplastischen Syndrome in verschiedene Subgruppen klassifiziert. Dies ermöglicht nicht nur die Vergleichbarkeit von Studien, sondern hat für den einzelnen Patienten auch prognostische Relevanz.

Erstmals wurde im Jahre 1982 durch die French-American-British(FAB) Co-operative Group die sog. FAB-Klassifikation für MDS eingeführt. Hier wurden fünf Subtypen ausschließlich morphologisch eingeteilt, um einerseits die Vergleichbarkeit zwischen verschiedenen Studien zu verbessern und andererseits, um die Suche nach prognostischen Kriterien zu erleichtern (Bennett et al. 1982). Es folgten aufgrund des Wissenszuwachses die World Health Organization (WHO) - Klassifikationen von 2001 und 2008, die zuletzt von der aktuellen Version von 2016 abgelöst wurden. 
Tabelle 1: WHO-Klassifikation Myelodysplastischer Syndrome (Arber et al. 2016, S.2400)

\begin{tabular}{|c|c|c|c|c|c|}
\hline Kategorie & $\begin{array}{l}\text { Dysplas-ti- } \\
\text { sche Rei- } \\
\text { hen }\end{array}$ & Zytopenien $^{1}$ & $\begin{array}{l}\text { Ringsidero- } \\
\text { blasten (\% der } \\
\text { erythroiden } \\
\text { Zellen) }\end{array}$ & $\begin{array}{c}\text { Blasten im Kno- } \\
\text { chenmark (KM) } \\
\text { und peripherem } \\
\text { Blut }(\mathrm{pB})\end{array}$ & $\begin{array}{c}\text { Karyotyp } \\
\text { (konventio- } \\
\text { nelle Bände- } \\
\text { rung) }\end{array}$ \\
\hline $\begin{array}{l}\text { MDS mit } \\
\text { Einliniendysplasie }\end{array}$ & 1 & 1 oder 2 & $<15 \% /<5 \% 2$ & $\begin{array}{l}\mathrm{KM}<5 \%, \\
\mathrm{pB}<1 \%, \\
\text { keine Auer- Stäb- } \\
\text { chen }\end{array}$ & $\begin{array}{l}\text { Alle, außer die } \\
\text { Kriterien für } \\
\text { MDS mit iso- } \\
\text { lierter del }(5 q) \\
\text { sind erfüllt }\end{array}$ \\
\hline $\begin{array}{l}\text { MDS mit } \\
\text { Mehrliniendysplasie }\end{array}$ & 2 oder 3 & $1-3$ & $<15 \% /<5 \% 2$ & $\begin{array}{l}\mathrm{KM}<5 \%, \\
\mathrm{pB}<1 \%, \\
\text { keine Auer- Stäb- } \\
\text { chen }\end{array}$ & $\begin{array}{l}\text { Alle, außer die } \\
\text { Kriterien für } \\
\text { MDS mit iso- } \\
\text { lierter del }(5 q) \\
\text { sind erfüllt }\end{array}$ \\
\hline \multicolumn{6}{|l|}{$\begin{array}{l}\text { MDS mit } \\
\text { Ringsideroblasten }\end{array}$} \\
\hline $\begin{array}{l}\text { MDS mit } \\
\text { Ringsideroblasten und } \\
\text { Einliniendysplasie }\end{array}$ & 1 & 1 oder 2 & $\geq 15 \% / \geq 5 \% 2$ & $\begin{array}{l}\mathrm{KM}<5 \%, \\
\mathrm{pB}<1 \%, \\
\text { keine Auer- Stäb- } \\
\text { chen }\end{array}$ & $\begin{array}{l}\text { Alle, außer die } \\
\text { Kriterien für } \\
\text { MDS mit iso- } \\
\text { lierter del }(5 q) \\
\text { sind erfüllt }\end{array}$ \\
\hline $\begin{array}{l}\text { MDS mit } \\
\text { Ringsideroblasten und } \\
\text { Mehrliniendysplasie }\end{array}$ & 2 oder 3 & $1-3$ & $\geq 15 \% / \geq 5 \%{ }^{2}$ & $\begin{array}{l}\mathrm{KM}<5 \%, \\
\mathrm{pB}<1 \%, \\
\text { keine Auer- Stäb- } \\
\text { chen }\end{array}$ & $\begin{array}{l}\text { Alle, außer die } \\
\text { Kriterien für } \\
\text { MDS mit iso- } \\
\text { lierter del }(5 q) \\
\text { sind erfüllt }\end{array}$ \\
\hline MDS mit del(5q) & $1-3$ & $1-2$ & Irrelevant & $\begin{array}{l}\mathrm{KM}<5 \%, \\
\mathrm{pB}<1 \%, \\
\text { keine Auer- Stäb- } \\
\text { chen }\end{array}$ & $\begin{array}{l}\operatorname{del}(5 q) \text { isoliert } \\
\text { oder mit } 1 \text { an- } \\
\text { deren Anomalie } \\
\text { außer } \\
-7 / \operatorname{del}(7 q)\end{array}$ \\
\hline \multicolumn{6}{|l|}{ MDS mit Blastenexzess } \\
\hline $\begin{array}{l}\text { MDS mit Blastenexzess } \\
\text { (1) }\end{array}$ & $0-3$ & $1-3$ & Irrelevant & $\begin{array}{l}\text { KM 5-9 \% oder } \\
\text { pB 2-4\%, keine } \\
\text { Auer-Stäbchen }\end{array}$ & Irrelevant \\
\hline $\begin{array}{l}\text { MDS mit Blastenexzess } \\
\text { (2) }\end{array}$ & $0-3$ & $1-3$ & Irrelevant & $\begin{array}{l}\text { BM } 10-19 \% \text { oder } \\
\text { pB 5-19\% oder } \\
\text { Auer- Stäbchen }\end{array}$ & Irrelevant \\
\hline \multicolumn{6}{|l|}{ MDS, unklassifizierbar } \\
\hline $\begin{array}{l}\text { mit } 1 \% \text { peripheren Blas- } \\
\text { ten }\end{array}$ & $1-3$ & $1-3$ & Irrelevant & $\begin{array}{l}\mathrm{KM}<5 \% \\
\mathrm{pB}=1 \% 3, \\
\text { keine Auer- Stäb- } \\
\text { chen }\end{array}$ & Irrelevant \\
\hline $\begin{array}{l}\text { Mit Einliniendysplasie } \\
\text { und Panzytopenie }\end{array}$ & 1 & 3 & Irrelevant & $\begin{array}{l}\mathrm{KM}<5 \%, \\
\mathrm{pB}<1 \%, \\
\text { keine Auer- Stäb- } \\
\text { chen }\end{array}$ & $\begin{array}{l}\text { Alle, außer die } \\
\text { Kriterien für } \\
\text { MDS mit iso- } \\
\text { lierter del }(5 q) \\
\text { sind erfüllt }\end{array}$ \\
\hline $\begin{array}{l}\text { Auf der Grundlage defi- } \\
\text { nierender zytogenetischer } \\
\text { Veränderungen }\end{array}$ & 0 & $1-3$ & $<15 \% 4$ & $\begin{array}{l}\mathrm{KM}<5 \%, \\
\mathrm{pB}<1 \%, \\
\text { keine Auer Stäb- } \\
\text { chen }\end{array}$ & $\begin{array}{l}\text { MDS- } \\
\text { definierende } \\
\text { Abnormalität }\end{array}$ \\
\hline
\end{tabular}

Legende: ${ }^{1}$ Zytopenien definiert durch: Hämoglobin $<10 \mathrm{~g} / \mathrm{dl}$, Thrombozyten $<100 \times 10^{9} / \mathrm{L}$, neutrophile Granulozyten $<1,8 \times 10^{9} / \mathrm{L}$, Monozyten im $p B<1 \times 10^{9} / \mathrm{L} ;{ }^{2}$ falls SF3B1 mutiert; ${ }^{3} 1 \%$ periphere Blasten müssen $z$ 2 verschiedenen Zeitpunkten beurteilt werden; ${ }^{4}$ Fälle mit $\geq 15 \%$ Ringsideroblasten haben definitionsgemäß eine signifikante Dyserythropoese und sind daber MDS mit Ringsideroblasten und Einliniendysplasie; 


\subsection{Prognoseeinschätzung}

Für die Einschätzung der Prognose eines Patienten nach Diagnose eines myelodysplastischen Syndroms und zur Auswahl der geeigneten Therapie haben sich heute das International Prognostic Scoring System (IPSS) und das Revised International Prognostic Scoring System (IPSS-R) für die initiale Evaluation etabliert. Beide Scores sind für eine Risikostratifizierung bei Diagnosestellung evaluiert. Im IPSS erfolgt die Einteilung dabei in insgesamt vier Risikogruppen anhand der Anzahl der Zytopenien im peripheren Blut, des Blastenanteils im Knochenmark und der zytogenetischen Veränderungen (Greenberg et al. 1997).

Tabelle 2: International Prognostic Scoring System (IPSS) (Greenberg et al. 1997, S. 2085)

\begin{tabular}{|l|c|c|c|c|c|}
\hline Score & 0 & 0,5 & 1 & 1,5 & 2 \\
\hline Blasten im KM (\%) & $<5$ & $5-10$ & - & $11-20$ & $21-30$ \\
\hline Karyotyp $^{1}$ & günstig & intermediär & ungünstig & - & - \\
\hline Zahl der Zytopenien $^{2}$ & $0 / 1$ & $2 / 3$ & - & - & - \\
\hline \multicolumn{2}{|c|}{ Risiko-Kategorie } & \multicolumn{3}{|c|}{ Risiko-Score } \\
\hline Low & & 0 \\
\hline intermediate I & & $0,5-1$ \\
\hline intermediate II & & \multicolumn{3}{c|}{$\geq 2,5-2$} \\
\hline High
\end{tabular}

Legende: '1günstig: normal, $-Y$, del(5q), del(20q); ungünstig: komplex $\geq 3$ Anomalien) oder Aberrationen auf Chromosom 7; intermediär: alle anderen Aberrationen

${ }^{2}$ Hämoglobin $<10 \mathrm{~g} / \mathrm{dl}$, Neutropbile Granulozyten $<1,8 / \mathrm{nl}$, Thrombozyten $<100$ / nl.

Der IPSS-R trägt dem Zuwachs an Wissen über die Prognose verschiedener zytogenetischer Veränderungen Rechnung und teilt Patienten in insgesamt fünf Risikogruppen auf. Dabei liegt eine stärkere Gewichtung auf der Zytogenetik, die Grenzwerte für die Zytopenien wurden entsprechend den Studienergebnissen angepasst, und der Blastenanteil im Knochenmark diversifiziert (Greenberg et al. 2012; Schanz et al. 2012). 
Tabelle 3: Revised International Prognostic Scoring System (IPSS-R)

(Greenberg et al. 2012 S. 2457-2458)

\begin{tabular}{|c|c|c|c|c|c|c|c|}
\hline Score & 0 & 0,5 & 1,0 & 1,5 & 2,0 & 3,0 & 4,0 \\
\hline Zytogenetische Risikogruppe & sehr gut & - & gut & - & $\begin{array}{l}\text { inter- } \\
\text { mediär }\end{array}$ & $\begin{array}{l}\text { un-güns- } \\
\text { tig }\end{array}$ & $\begin{array}{l}\text { Sehr un- } \\
\text { günstig }\end{array}$ \\
\hline Blasten im KM (\%) & $\leq 2$ & - & $>2-<5$ & - & $5-10$ & $>10$ & - \\
\hline Hämoglobin (g/dl) & $\geq 10$ & - & $8-<10$ & $<8$ & - & - & - \\
\hline Thrombozyten $\left(\mathrm{x} 10^{3} / \mathrm{l}\right)$ & $\geq 100$ & $50-<100$ & $<50$ & - & - & - & - \\
\hline $\begin{array}{l}\text { ANC }\left(x 10^{3} / \mathrm{l}\right) \\
\text { (absolute Neurophilenzahl) }\end{array}$ & $\geq 0,8$ & $<0,8$ & - & - & - & - & - \\
\hline \multicolumn{3}{|c|}{ Risiko-Kategorie } & \multicolumn{5}{|c|}{ Risiko-Score } \\
\hline \multicolumn{3}{|l|}{ very low } & \multicolumn{5}{|c|}{$\leq 1,5$} \\
\hline \multicolumn{3}{|l|}{ Low } & \multicolumn{5}{|c|}{$>1,5-3$} \\
\hline \multicolumn{3}{|l|}{ Intermediate } & \multicolumn{5}{|c|}{$>3-4,5$} \\
\hline \multicolumn{3}{|l|}{ High } & \multicolumn{5}{|c|}{$>4,5-6$} \\
\hline \multicolumn{3}{|l|}{ very high } & \multicolumn{5}{|c|}{$>6$} \\
\hline
\end{tabular}

Legende: sehrgut: $-Y$, del(11q);

gut: Normal, del(5q), del(12p), del(20q), double including del(5q);

intermediär: $\operatorname{del}(7 q),+8,+19, i(17 q)$, any other single or double independent clones;

ungünstig: -7, inv (3)/t(3q)/del(3q), double including -7/del(7q), complex: 3 abnormalities;

sebr ungünstig : Complex: $>3$ abnormalities; - bedeutet nicht zutreffend

Für die Prognoseeinschätzung unter fortschreitender Erkrankung oder unter Therapie exsistiert das WHO-adaptierte Prognostic Scoring System (WPSS). Hier wird zusätzlich der Transfusionsbedarf des Patienten als dynamischer Parameter berücksichtigt (Malcovati et al. 2011; Greenberg et al. 2017). 
Tabelle 4: WHO-adaptiertes Prognostic Scoring System (WPSS)

(Malcovati et al. 2011, S. 1437)

\begin{tabular}{|c|c|c|c|c|}
\hline Score & 0 & 1 & 2 & 3 \\
\hline WHO-Typ & $\begin{array}{l}\text { RCUD/RARS/MDS } \\
\text { mit isolierter } \operatorname{del}(5 \mathrm{q})\end{array}$ & RCMD & RAEB-I & RAEB-II \\
\hline $\begin{array}{l}\text { zytogenetische Ri- } \\
\text { sikogruppe }^{1}\end{array}$ & günstig & intermediär & Ungünstig & - \\
\hline Transfusion $^{2}$ & Nein & $\mathrm{Ja}$ & & \\
\hline \multicolumn{2}{|c|}{ Risiko-Kategorie } & & \multicolumn{2}{|c|}{ Risiko-Score } \\
\hline \multicolumn{2}{|l|}{ very low } & & \multicolumn{2}{|c|}{0} \\
\hline \multicolumn{2}{|l|}{ Low } & & \multicolumn{2}{|c|}{1} \\
\hline \multicolumn{2}{|l|}{ Intermediate } & & \multicolumn{2}{|c|}{2} \\
\hline \multicolumn{2}{|l|}{ High } & & \multicolumn{2}{|c|}{ 3-4 } \\
\hline \multicolumn{2}{|l|}{ very high } & & \multicolumn{2}{|c|}{ 5-6 } \\
\hline
\end{tabular}

Legende: 'gü̈nstig: normal, 5q-, 20q-, -Y; ungünstig: komplexer Karyotyp, Chromosom 7-Anomalien; intermediär: alle anderen Aberrationen

${ }^{2}$ Transfusionsbedarf: Männer $\mathrm{H} b<9 g / d l$, Frauen $\mathrm{H} b<8 g / d l$

\subsection{Therapie}

Für die Auswahl an Therapieoptionen hat es sich als sinnvoll erwiesen, zwei Risikogruppen zu bilden.

Niedrig-Risiko-Patienten haben in der Regel einen Risikoscore nach IPSS von low oder intermediate-1; einen IPSS-R very low, low oder intermediate; oder einen WPSS very low, low oder intermediate (Greenberg et al. 2017).

Für diese Patienten bestehen Therapiemöglichkeiten mit Immunmodulatoren (Lenalidomid) bei Nachweis eines MDS mit Deletion(5q) alleine oder in Kombination mit einer weiteren zytogenetischen Veränderung. Ausgenommen sind hierbei allerdings die Monosomie 7 und die Deletion(7q) als zusätzliche Anomalie (Greenberg et al. 2017).

Darüber hinaus können hämatopoetische Wachstumsfaktoren eingesetzt werden, und unter bestimmten Voraussetzungen können auch immunsuppressive Therapien eine Verbesserung der Blutbildparameter erreichen (Malcovati et al. 2013).

Hoch-Risiko-Patienten haben in der Regel einen Risikoscore high oder intermediate-2 nach IPSS oder intermediate, high oder very high nach IPSS-R oder high oder very high nach WPSS (Greenberg et al. 2017). 
Für diese Patienten existieren zugelassene Therapien mit den hypomethylierenden Medikamenten Azacitidine und Decitabine. Intensive Chemotherapien analog der akuten myeloischen Leukämie, ggf. gefolgt von einer allogenen Stammzelltransplantation als nach wie vor einzig kurativer Option, sind hier ebenfalls für die betroffenen Patienten zu evaluieren. Bei der Entscheidung für eine intensive Therapie spielen zusätzlich zum Risikoscore patientenimmanente Faktoren, wie das Alter, eventuelle Komorbiditäten, der Performance- Status und auch der Patientenwille, eine entscheidende Rolle (Greenberg et al. 2017).

Alle Patienten sollten außerdem nach Bedarf supportive Maßnahmen erhalten. Dazu gehören die Substitution mit Blutprodukten, die frühzeitige Behandlung von Infektionen und eine Eisenchelation zur Vermeidung einer sekundären Eisenüberladung bei chronischem Transfusionsbedarf (Greenberg et al. 2017).

\subsection{Zytogenetik}

Die Zytogenetik ist der entscheidende Parameter bei myelodysplastischen Syndromen. Sie ist obligat für die Diagnostik und Einordnung in die Erkrankungssubgruppe der WHOKlassifikation, da seit deren Revision in 2016 in der Kategorie der unklassifizierbaren MDS sogar eine eigene Subgruppe mit MDS-definierenden chromosomalen Veränderungen existiert. So werden nun unklare periphere Zytopenien bei fehlenden Dysplasiezeichen und $<5 \%$ Blasten im Knochenmark bei Nachweis bestimmter chromosomaler Aberrationen (z. B. einer Monosomie 7, einer Deletion (7q) oder Deletion (5q), sowie weiteren typischen Deletionen und Translokationen) als unklassifizierbares MDS diagnostiziert (Arber et al. 2016), wo bisher zur Diagnose MDS der Nachweis von Dysplasiezeichen unabdingbar war. Zum anderen besteht bereits seit Einführung der WHO-Klassifikation in 2001 die Subgruppe der MDS mit Deletion (5q).

Die besondere Relevanz der Zytogenetik liegt aber in ihrer validierten Aussagekraft bezüglich der Prognose hinsichtlich Gesamtüberleben und Risiko der Entwicklung einer akuten myeloischen Leukämie, die in vielen Arbeiten untersucht und bestätigt wurde (Yunis et al. 1988; Sanz et al. 1989; Toyama et al. 1993; Greenberg et al. 1997; Solé et al. 2000; Haase et al. 2007; Schanz et al. 2012; Greenberg et al. 2012) und damit auch für die Auswahl einer potentiellen Therapie.

Zytogenetische Veränderungen sind bei myelodysplastischen Syndromen schon lange bekannt und werden hinsichtlich ihrer Häufigkeit und prognostischen Relevanz systematisch untersucht. Die Frequenz eines abnormalen Karyotyps in 43,5\% der Fälle zusammen mit der Tendenz zu einem kürzeren Überleben beschreiben z. B. Tricot et al. (1985) in einer Untersuchung an 85 Patienten mit MDS. Die Häufigkeit chromosomaler Aberrationen variieren dabei in den verschiedenen Studien abhängig vom untersuchten Kollektiv zwischen 39\% (Jacobs et al. 1986) und 73\% (Yunis et al. 1988). Bei primären MDS lassen sich bei ca. 50\% der Patienten zytogenetische Veränderungen nachweisen (Solé et al. 2005; Haase et al. 2007; 
Pozdnyakova et al. 2008). Diese Rate steigt bei den therapieassoziierten sekundären MDS auf bis zu 80\% an (Toyama et al. 1993; Haase et al. 1995; Solé et al. 2000).

In den letzten Jahren kommt auch dem Nachweis genetischer Mutationen zunehmende Bedeutung zu. Viele der nachgewiesenen Mutationen haben ebenfalls prognostische Relevanz und sind zum Beispiel mit einem kürzeren AML-freien Überleben assoziiert (Bejar et al. 2011). Bei 80-90\% der MDS Patienten lassen sich solche Mutationen nachweisen (Haferlach et al. 2014) und die häufigsten sind Mutationen in den Genen SF3B1, TET2, SRSF2 und ASXL1 bei mehr als 10\% der MDS-Fälle. (Papaemmanuil et al. 2013). Eine Integration in die klinisch derzeit gängigen Prognose-Scores konnte jedoch bisher nicht erfolgen.

Chromosomale Aberrationen können sowohl als numerische Veränderungen, wie Monosomien und seltener Trisomien, als auch als strukturelle Veränderungen imponieren (z. B. Inversionen und Deletionen). Seltener kommen balancierte Translokationen vor. Zytogenetische Auffälligkeiten treten aber nicht nur isoliert, sondern auch in Kombination oder im Rahmen eines komplexen Karyotyps (>3 Anomalien) auf (Toyama et al. 1993; Haase et al. 2007; Bejar et al. 2011; Schanz et al. 2012).

Verkompliziert wird ihre prognostische Einordnung dabei durch die Tatsache, dass alle Veränderungen isoliert oder mit einer oder mehreren weiteren Anomalien auftreten können. Außerdem können bei Erstdiagnose bereits mehrere Anomalien nachweisbar sein oder erst im Krankheitsverlauf auftreten. Dabei beeinträchtigt die Zunahme an chromosomalen Veränderungen die Prognose der Patienten erheblich (Greenberg et al. 1997; Schanz et al. 2012; Greenberg et al. 2012) und das Hinzutreten neuer Veränderungen im Verlauf der Erkrankung markiert oftmals den Progress (Jabbour et al. 2013). Insgesamt ist die genetische Heterogenität der myelodysplastischen Syndrome hoch; eine Vielzahl an Veränderungen ist in der Literatur beschrieben (Solé et al. 2005; Haase et al. 2007).

Als die am häufigsten auftretenden Anomalien werden übereinstimmend in absteigender Häufigkeit der Verlust des langen Armes am Chromosom 5 (5q-), der vollständige oder partielle Verlust eines Chromosom 7 (-7/7q-), die Trisomie $8(+8)$, die Deletion (20q), sowie der Verlust des Y-Chromosoms beschrieben (Nowell 1982; Jacobs et al. 1986; Haase et al. 1995; Solé et al. 2000; Haase et al. 2007; Pozdnyakova et al. 2008; Schanz et al. 2012; Greenberg et al. 2012). Dabei wurde die häufigste zytogenetische Veränderung bei MDS, die 5qDeletion, bereits 1974 in einer Fallserie als dysplastische Knochenmarkserkrankung mit speziellen klinischen und morphologischen Besonderheiten beschrieben (van den Berghe et al. 1974). Gemeinsam machen diese Veränderungen alleine oder in Kombination mit anderen Anomalien sowie in Form eines komplexen Karyotyps etwa 70\% der chromosomalen Veränderungen bei MDS aus (Solé et al. 2000). Darüber hinaus kommt jedoch eine Vielzahl weiterer chromosomaler Veränderungen bei MDS vor, deren prognostische Einschätzung aufgrund der Seltenheit ihres Auftretens schwierig ist.

Mit der Entwicklung des IPSS wurde es möglich, 86\% der einzeln oder in Kombination auftretenden Veränderungen hinsichtlich ihrer Prognose zu klassifizieren (Greenberg et al. 
1997) und mit Entwicklung des IPSS-R gelang dies noch einmal für weitere 5\%. Insgesamt gelingt heute also für $91 \%$ der bei MDS gefunden zytogenetischen Veränderungen eine prognostische Einordnung (Schanz et al. 2012). Sowohl im IPSS als auch im IPSS-R befinden sich innerhalb der Gruppe mit intermediärem Risiko die seltenen zytogenetischen Einzelanomalien, über deren Prognose aufgrund ihrer geringen Fallzahl bisher keine prognostische Aussage möglich ist. Ob sich unter ihnen jedoch noch prognostisch klar definierbare Anomalien befinden, ist bisher unklar.

\subsection{Fragestellung der Arbeit}

Wie oben dargestellt, sind seltene Einzelchromosomenanomalien hinsichtlich ihrer prognostischen Einordnung problematisch. Es gibt bisher für einen Anteil von 9\% der Aberrationen keine prognostische Untersuchung hinsichtlich der Überlebenszeit und der AML-freien Überlebenszeit. Bisher befinden sich diese Veränderungen in der Kategorie des intermediären Risikos nach IPSS-R. Ob diese Einordnung für jede der auftretenden Veränderungen zutrifft, ist bisher nicht bekannt. Vielmehr stellt die prognostische Kategorie „Intermediär“ ein heterogenes Gemisch prognostisch unterschiedlicher Anomalien dar, welche nur in ihrer Gesamtheit aufgrund statistischer Effekte eine scheinbar intermediäre Prognose haben.

Diese Arbeit untersucht daher innerhalb einer internationalen Datenbank für primäre myelodysplastische Syndrome mit 7012 Fällen

1. die absolute Häufigkeit von seltenen Einzelchromosomenanomalien

2. die prognostische Relevanz der identifizierten seltenen Aberrationen auf die Überlebenszeit und das AML-freie Überleben,

3. erörtert mögliche Konsequenzen hinsichtlich der Einordnung einzelner Veränderungen in die Prognosegruppen des IPSS-R. 


\section{Material und Methoden}

\subsection{Patientenkollektiv und Datenerhebung}

Die vorliegende Arbeit bedient sich für ihre retrospektive Analyse der Datenbank der IWGPM (International Working Group for the Prognosis of MDS). Aus dieser Datenbank stehen 7012 anonymisierte Fälle von primären MDS-Patienten zur Verfügung, die ausschließlich supportiv behandelt wurden. In der Datenbank wurden sowohl Basisdaten (Alter und Geschlecht) und klinische Parameter als auch zytogenetische Befunde dokumentiert. Insgesamt konnten 382 Patienten mit Einzelchromosomenanomalien anhand ihres zytogenetischen Befundes extrahiert werden. Diese Fälle wurden in eine Excel-Datenbank (Microsoft Office Excel 2007) überführt.

Sämtliche verarbeiteten klinischen Daten, zytogenetischen Befunde und Daten der Verlaufsbeobachtung standen in der verwendeten Datenbank zur Verfügung. Die Daten der Kontrollgruppe für die multivariate Analyse entsprechen der Kontrollgruppe aus der zytogenetischen Analyse von Schanz et al. (2012) und wurden von Frau Dr. med. Schanz zur Verfügung gestellt. Für die Kontrollgruppe wurden Fälle mit normalem Karyotyp verwendet, um hier die entsprechenden Analysen im Vergleich zu Patienten ohne Chromosomenanomalien durchführen zu können. Zusätzliche Daten wurden nicht erhoben, es erfolgte kein Patientenkontakt und die Richtlinien für Datenschutz und Schweigepflicht wurden befolgt.

In der univariaten Analyse der Überlebenszeit konnten n=264 Fälle mit seltenen Anomalien berücksichtigt werden, für die univariate Analyse der AML-freien Überlebenszeit $n=207$ Fälle. Für die multivariate Berechnung der Überlebenszeit ergibt sich durch die Hinzunahme der Kontrollgruppe mit normalem Karyotyp (NOR) mit n=604 Fällen eine Gesamtzahl von 857 Fällen. Die multivariate Analyse hinsichtlich des AML-freien Überlebens wurde mit 744 Fällen durchgeführt. Davon waren 517 Fälle der Kontrollgruppe.

\subsubsection{Herkunft der untersuchten Fälle}

Das untersuchte Kollektiv setzt sich aus Patienten aus insgesamt 16 internationalen Zentren zusammen. Bei 382 eingeschlossenen Fällen stammen 28,5\% der Fälle aus der Spanischen MDS-Studiengruppe, 19,1\% aus dem MD. Anderson Cancer Center (Texas, USA) 11,3\% aus der Datenbank des International MDS Risk Analysis Workshop (IMRAW), 8,9\% aus Frankreich, 6,5\% aus Pavia (Italien) 5,5\% aus Wien (Österreich), 4,2\% aus Düsseldorf (Deutschland), 3,7\% aus Japan, 2,9\% aus Italien (außer Pavia), 2,6\% aus Cleveland (Ohio, USA) 2,4\% Dundee (Schottland), 2,1\% aus Brasilien, 1,3\% aus den Niederlanden, 0,5\% aus Tschechien sowie jeweils 0,3\% aus Freiburg (Deutschland) und Innsbruck (Österreich). Für die multivariate Analyse wurden die verschiedenen Zentren in Gruppen zusammengefasst, 
sodass alle Fälle europäischer Zentren eine Gruppe bildeten (EUR), die Fälle nordamerikanischer Zentren (USA) eine weitere und alle übrigen Zentren eine dritte Gruppe (OTH).

\subsubsection{Diagnosestellung}

Erfasst wurden in der Datenbank an den jeweiligen Zentren ausschließlich Patienten mit primären myelodysplastischen Syndromen. Die Einteilung in die verschiedenen Subtypen erfolgte anhand der FAB-Klassifikation und für die später aufgenommenen Fälle größtenteils zusätzlich anhand der jeweils gültigen WHO-Klassifikation von 2001 und 2008, da zum Zeitpunkt der Diagnose der verwendeten Fälle die entsprechend neueren Klassifikationen noch nicht zur Verfügung standen.

Tabelle 5: FAB-Klassifikation Myelodysplastischer Syndrome

(Bennett et al. 1982 S. 195, 197)

\begin{tabular}{|c|c|c|c|}
\hline MDS-Subtypen & $\begin{array}{l}\text { Befunde im } \\
\text { peripheres Blut }(\mathrm{pB})\end{array}$ & $\begin{array}{l}\text { Befunde im } \\
\text { Knochenmark } \\
(\mathrm{KM})\end{array}$ & weitere Befunde \\
\hline Refraktäre Anämie (RA) & $\begin{array}{l}\text { Anämie, } \\
<1 \% \text { Blasten }\end{array}$ & $<5 \%$ Blasten & $\begin{array}{l}\text { pB: Retikulozytopenie, Dys- } \\
\text { erythropoese } \\
\text { KM: Normo- oder } \\
\text { Hyperzellularität }\end{array}$ \\
\hline $\begin{array}{l}\text { Refraktäre Anämie mit } \\
\text { Ringsideroblasten } \\
\text { (RARS) }\end{array}$ & $\begin{array}{l}\text { Anämie, } \\
<1 \% \text { Blasten }\end{array}$ & $<5 \%$ Blasten & $\begin{array}{l}\text { pB: wie RA } \\
\text { KM: Normo- oder Hyper- } \\
\text { zellularität, } \\
>15 \% \text { Ringsideroblasten }\end{array}$ \\
\hline $\begin{array}{l}\text { Refraktäre Anämie mit } \\
\text { Blasten-Exzess (RAEB) }\end{array}$ & $\begin{array}{l}\text { Zytopenien, } \\
<5 \% \text { Blasten }\end{array}$ & $5-20 \%$ Blasten & $\begin{array}{l}\text { pB: Dysplasiezeichen in al- } \\
\text { len drei Zellreihen } \\
\text { KM: Hyperzellularität, Rei- } \\
\text { fungsstörungen, } \\
\pm \text { Ringsideroblasten }\end{array}$ \\
\hline $\begin{array}{l}\text { Chronische } \\
\text { myelomonozytäre } \\
\text { Leukämie (CMML) }\end{array}$ & $\begin{array}{l}>1 \times 10^{9} \text { Monozyten } / \mathrm{L} \\
<5 \% \text { Blasten }\end{array}$ & $\leq 20 \%$ Blasten & $\begin{array}{l}\text { KM: wie RAEB, zusätzlich } \\
\text { erhöhte Anzahl an } \\
\text { Promonozyten }\end{array}$ \\
\hline $\begin{array}{l}\text { RAEB in Transformation } \\
\text { (RAEB-T) }\end{array}$ & $\geq 5 \%$ Blasten & $21-30 \%$ Blasten & Auerstäbchen \\
\hline
\end{tabular}


Tabelle 6: WHO-Klassifikation Myelodysplastischer Syndrome (Vardiman et al. 2009 S.943)

\begin{tabular}{|c|c|c|}
\hline MDS-Subtypen & $\begin{array}{l}\text { Befunde im } \\
\text { peripheren Blut }\end{array}$ & $\begin{array}{l}\text { Befunde im } \\
\text { Knochenmark }\end{array}$ \\
\hline $\begin{array}{l}\text { Refraktäre Zytopenie mit } \\
\text { unilineärer Dysplasie (RCUD) } \\
\text { - Refraktäre Anämie (RA) } \\
\text { - Refraktäre Neutropenie (RN) } \\
\text { - Refraktäre Thrombozytopenie (RT) }\end{array}$ & $\begin{array}{l}\text { Uni- oder Bizytopenie }{ }^{1} \text {, } \\
\text { keine Blasten oder }<1 \% 0^{2}\end{array}$ & $\begin{array}{l}\text { Dysplasien in } \geq 10 \% \text { der } \\
\text { Zellen einer Reihe, } \\
<5 \% \text { Blasten, } \\
<15 \% \text { Ringsideroblasten }\end{array}$ \\
\hline $\begin{array}{l}\text { Refraktäre Anämie mit } \\
\text { Ringsideroblasten (RARS) }\end{array}$ & Anämie, keine Blasten & $\begin{array}{l}\text { ausschließlich } \\
\text { Dyserythropoese, } \\
<5 \% \text { Blasten, } \\
\geq 15 \% \text { Ringsideroblasten }\end{array}$ \\
\hline $\begin{array}{l}\text { Refraktäre Zytopenie mit } \\
\text { multilineären Dysplasien (RCMD) }\end{array}$ & $\begin{array}{l}\text { Zytopenie(n), } \\
\text { keine Blasten oder }<1 \% 0^{2}, \\
\text { keine Auer-Stäbchen } \\
<1 \times 10^{9} / \text { L Monozyten, }\end{array}$ & $\begin{array}{l}\text { Dysplasien in } \geq 10 \% \text { der } \\
\text { Zellen in } \geq 2 \text { Zellreihen, } \\
<5 \% \text { Blasten, } \\
\pm 15 \% \text { Ringsideroblasten, } \\
\text { keine Auer-Stächen }\end{array}$ \\
\hline $\begin{array}{l}\text { Refraktäre Anämie mit } \\
\text { Blastenexzess-I (RAEB-I) }\end{array}$ & $\begin{array}{l}\text { Zytopenie(n), <5\% Blasten }{ }^{2} \text {, } \\
\text { keine Auer-Stäbchen, } \\
<1 \times 10^{9} / \text { L Monozyten }\end{array}$ & $\begin{array}{l}\text { uni- oder multilineäre Dys- } \\
\text { plasien, 5-9\% Blasten }{ }^{2} \text {, } \\
\text { keine Auer-Stäbchen }\end{array}$ \\
\hline $\begin{array}{l}\text { Refraktäre Anämie mit } \\
\text { Blastenexzess-II (RAEB-II) }\end{array}$ & $\begin{array}{l}\text { Zytopenie(n), 5-19\% Blasten, } \\
\text { Auer-Stäbchen } \pm^{3} \\
<1 \times 10^{9} / \text { L Monozyten, }\end{array}$ & $\begin{array}{l}\text { uni- oder multilineäre Dys- } \\
\text { plasien, } \\
\text { 10-19\% Blasten, } \\
\text { Auer-Stäbchen } \pm^{3}\end{array}$ \\
\hline Unklassifizierbares MDS (MDS-U) & Zytopenien, $\leq 1 \%$ Blasten $^{2}$ & $<5 \%$ Blasten \\
\hline MDS mit isolierter del $(5 q)$ & $\begin{array}{l}\text { Anämie, Thrombozyten oft } \\
\text { normal oder vermehrt, keine } \\
\text { Blasten oder }<1 \%\end{array}$ & $\begin{array}{l}\text { Normale oder vermehrte } \\
\text { Megakaryozyten mit hypo- } \\
\text { lobulierten Kernen, } \\
<5 \% \text { Blasten, isolierte } \\
\text { del(5q) Anomalie, } \\
\text { keine Auer-Stäbchen }\end{array}$ \\
\hline
\end{tabular}

Legende: ${ }^{1}$ Im Falle einer Panzytopenie erfolgt die Zuordnung in die Gruppe MDS-U.

${ }^{2}$ Bei $<5 \%$ Blasten im KM und 2-4\% Blasten im $p B$ erfolgt die Einteilung in die Gruppe RAEB-I. Patienten mit $1 \%$ Blasten im $p B$ in den Gruppen RCUD und RCMD werden als MDS-U klassifiziert.

${ }^{3}$ Bei Vorliegen von Auerstäbchen, $<5 \%$ Blasten im $p B$ und $<10 \%$ Blasten im KM erfolgt die Klassifikation als RAEB-II. 


\subsubsection{Basisdaten und Klinische Daten}

Angaben zum Alter bei Diagnosestellung standen für 71,2\% $(n=272)$ und für das Geschlecht für 71,5\% ( $\mathrm{n}=273)$ der Patienten zur Verfügung. Werte für Hämoglobin $(\mathrm{Hb})$, die absolute Neutrophilenzahl (ANC) und die Thrombozytenzahl (PLT) lagen bei 71,2\% ( $\mathrm{n}=272)$ der Patienten vor, die Anzahl der Blasten im Knochenmark (BLAKM) waren bei 66,2\% ( $\mathrm{n}=253)$ und die Gesamtgranulozytenzahl (WBC) bei 63,9\% $(n=244)$ ersichtlich. Blasten im peripheren Blut (BLAP) waren bei 43,2\% ( $\mathrm{n}=165)$, die Anzahl der analysierten Metaphasen (MEP) bei 37,4\% (n=143) und die Monozytenzahl (MONO) bei 26,7\% $(n=102)$ der Patienten angegeben.

\section{$\underline{\text { Hämoglobin }}$}

Der Hämoglobinwert wurde in $\mathrm{g} / \mathrm{dl}$ angegeben.

\section{Leukozyten/Granulozyten}

Die Zahl der Leukozyten wurde in $10^{3} / \mu$ gemessen, die Anzahl der Neutrophilen Granulozyten (ANC) als Teilmenge der Leukozyten ebenfalls.

\section{Monozyten}

Die Monozyten als Teilmenge der Leukozyten wurden ebenfalls in $10^{3} / \mu$ gezählt.

\section{Thrombozytenzahl}

Thrombozytenwerte wurden ebenfalls in $10^{3} / \mu$ l angegeben.

\section{$\underline{\text { Blasten }}$}

Der Anteil an Blasten im Knochenmark und im peripheren Blut wurde in Prozent (\%) der ausgezählten mononukleären Zellen angegeben.

\section{Überlebenszeit/Sekundäre AML}

Die Überlebenszeit (ÜLZ) und die Zeit bis zum Auftreten einer AML (AFS) wurden in Monaten angegeben. Dabei begann die Zeit der klinischen Verlaufsbeobachtung jeweils mit der Durchführung der ersten Zytogenetik. 
Im Minimum wurden 4, im Maximum 49 und im Median 20 Metaphasen nach Giemsabandenfärbung analysiert. Damit sind internationale Qualitätsstandards erfüllt.

\subsubsection{Klinische Verlaufsbeobachtung (Follow-up)}

In der Verlaufsbeobachtung wurden die Ereignisse „sekundäre akute myeloische Leukämie“ und Tod bzw. das Datum des letzten Patientenkontaktes erfasst.

\subsection{Zytogenetische Befunde}

\subsubsection{Definition zytogenetischer Befunde}

Die Erstellung der zytogenetischen Befunde erfolgte an den jeweiligen Zentren nach ISCN (International System for Human Cytogenetic Nomenclature) in der jeweils bei Diagnose geltenden Fassung (Shaffer et al. 2013), was die einheitliche Dokumentation des Karyotyps gewährleistete.

\subsubsection{Chromosomale Veränderungen}

Klonale zytogenetische Aberration

Gemäß Vorgabe des ISCN liegen klonale zytogenetische Aberrationen vor, wenn in mindestens zwei Metaphasen identische zusätzliche Chromosomen oder in mindestens drei Metaphasen identische strukturelle Veränderungen oder fehlende Chromosomen nachgewiesen werden.

\section{$\underline{\text { Einzelchromosomenaberration }}$}

Auftreten einer einzelnen Anomalie innerhalb eines Klons ohne zusätzliche Anomalien.

\section{Markerchromosom}

Als Markerchromosom gelten strukturell abnormale Chromosomen, von denen kein Teil als einem bestimmten Chromosom zugehörig identifiziert werden kann. Sie können in unterschiedlicher Größe und Form vorliegen.

\section{Derivative Chromosomen}

Hierbei handelt es sich um strukturell veränderte Chromosomen, die entweder durch den Austausch genetischen Materials zwischen zwei oder mehr Chromosomen oder durch multiple Aberrationen in einem einzelnen Chromosom zustande kommen. 
$\underline{\text { Translokation, balanciert }}$

Hierbei handelt es sich um den Austausch von genetischem Material zwischen zwei oder mehr Chromosomen. Formal handelt es hierbei um zwei Anomalien, da an zwei Chromosomen Veränderungen entstehen. Es kommt allerdings dabei nicht zu Verlust oder Zugewinn an genetischem Material, weshalb balancierte Translokationen als Einzelanomalien gewertet werden. Außerdem gehören balancierte Translokationen zu den seltenen Anomalien bei myelodysplastischen Syndromen. Und ihre bisherige Einordnung in die Prognosegruppe mit intermediärem Risiko macht diese Gruppe für die vorliegende Arbeit relevant.

\section{$\underline{\text { Monosomie }}$}

„Monosomie“ bezeichnet den Verlust eines vollständigen Chromosoms oder ganzer Chromosomenarme.

\section{$\underline{\text { Trisomie }}$}

„Trisomie“ bezeichnet den Zugewinn vollständiger Chromosomen oder ganzer Chromosomenarme.

\section{Duplikation}

Unter „Duplikation“ wird die Verdopplung von genetischem Material im bestehenden Chromosom verstanden, was zu einer Verlängerung des entsprechenden Armes führt.

\subsubsection{Verteilungstyp}

Die gefundenen seltenen Einzelchromosomenaberrationen bestanden entweder in allen oder nur in einem Teil der pro Patient analysierten Metaphasen. War dies der Fall, hatten die übrigen Metaphasen jeweils einen normalen Karyotyp (Mosaik).

\subsubsection{Definierte zytogenetische Gruppen}

Eine eigene Subgruppe wurde immer dann gebildet, wenn mindestens fünf Fälle mit den entsprechenden Veränderungen im Gesamtkollektiv auftraten und die klinischen Daten und Verlaufsdaten für die statistische Berechnung zur Verfügung standen. Dabei wurden auch verschiedene chromosomale Einzelaberrationen eines Chromosoms zusammengefasst, wenn ihre prognostische Bedeutung bisher nicht untersucht wurde. Dabei wurden alle Veränderungen an den untersuchten Chromosomen ausgenommen, deren Prognose bereits bekannt ist, wie z. B. die Deletion (5q). 
Für die Veränderungen am Chromosom 1 wurden zwei Gruppen gebildet, deren eine die Patienten mit partieller oder totaler Trisomie umfasst und die andere die Patienten mit Bildung eines derivativen Chromosoms (1;7), denn es war hier von unterschiedlichen Auswirkungen auf den Erkrankungsverlauf auszugehen.

Die gefundenen zytogenetischen Aberrationen wurden wie folgt zusammengefasst:

\section{$\underline{\text { ANY6/ANY11/ANY20 }}$}

Die Gruppe ANY beinhaltet sämtliche Einzelaberrationen an den Chromosomen 6, 11 und 20 mit Ausnahme balancierter Translokationen dieser Chromosomen, welche gesondert erfasst wurden.

\section{$\underline{\operatorname{DER}(1 ; 7)}$}

Nachweis einer unbalancierten Translokation $\mathrm{t}(1 ; 7)$, typischerweise mit den Bruchpunkten (q10;p10).

\section{$\underline{\text { MAR }}$}

Hier wurden alle Fälle mit einem zusätzlichen Markerchromosom zusammengefasst.

\section{$\underline{\mathrm{MO} 3 / \mathrm{MO} 9 / \mathrm{MO} 12 / \mathrm{MO} 14 / \mathrm{MO} 16 / \mathrm{MO} 18}$}

Diese zytogenetischen Gruppen enthalten jeweils Fälle mit partieller (Verlust des langen oder des kurzen Armes) oder totaler Monosomie des entsprechenden Chromosoms.

\section{MO13Q}

In dieser Gruppe befinden sich ausschließlich Patienten mit Verlust des langen Arms (q-) von Chromosom 13.

\section{$\underline{\mathrm{MO} 5}$}

Hier sind Fälle mit totalem Verlust von Chromosom 5 oder des kurzen Armes (p-) zusammengefasst. Der Verlust des langen Armes (5q-) wurde bereits als eigene Entität innerhalb der myelodysplastischen Syndrome beschrieben (van den Berghe et al. 1974) und bildet auch in der WHO-Klassifikation der myelodysplastischen Syndrome bereits seit 2001 eine eigene Subgruppe. 
Diese Gruppe umfasst ausschließlich Fälle mit Verlust eines X-Chromosoms.

\section{$\underline{\text { TR1Q }}$}

Diese Gruppe enthält Fälle mit Auftreten eines dritten Chromosoms 1, der Verdopplung des langen Armes (+1q) oder einer Duplikation des langen Armes von Chromosom 1 (dup1q).

$\underline{\text { TR21 }}$

In dieser Gruppe sind Fälle mit Auftreten eines dritten Chromosoms 21 (Trisomie) zusammengeführt.

\section{$\underline{\mathrm{TRB}}$}

Dieser Gruppe enthält alle Fälle mit balancierten Translokationen sämtlicher Chromosomen.

$\underline{\mathrm{OTH}}$

In dieser Gruppe befinden sich alle Anomalien, die so selten waren, dass keine eigene Gruppe gebildet werden konnte und auch keine andere sinnvolle Zusammenfassung möglich war.

\subsection{Statistische Methoden}

Die statistischen Berechnungen wurden mit Hilfe des Programms SPSS Statistics, Version 24 der Firma IBM corp. mit Sitz in Armonk im Bundesstaat New York, USA durchgeführt. Mittelwert, Median und Standardabweichung wurden mit den üblichen statistischen Methoden berechnet.

Für die Überlebenswahrscheinlichkeit erfolgte die Analyse nach der Methode von Kaplan und Meier (Kaplan und Meier 1958)

Eine Analyse war aus Gründen der statistischen Relevanz immer dann möglich, wenn eine bestimmte Aberration in mindestens fünf Fällen auftrat.

Für die Berechnung der Irrtumswahrscheinlichkeit zwischen kategorialen Variablen wurde der Chi-Quadrat-Test verwendet.

Die Irrtumswahrscheinlichkeit stetiger Variablen wurde mit Hilfe des Kruskal-Wallis-AnovaTests berechnet.

Unterschiede im Gesamt- oder AML-freien Überleben wurden mittels Log-Rank-Test (Peto et al. 1977) ermittelt. 
Die Berechnung der multivariaten Analysen erfolgte mittels Proportional-Hazard-Regressionsmodell (Cox 1972). Für die spätere Einordnung der Ergebnisse in die Risikogruppen des IPSS-R wurden als Variablen für die multivariate Analyse die entsprechenden Grenzwerte für Alter, Hb, ANC, Thrombozyten und Blasten im Knochenmark des IPSS-R eingefügt. Außerdem erfolgte die zusätzliche Integration einer Vergleichsgruppe mit normalem Karyotyp und bereits bekannter Überlebenszeit und AML-freier Überlebenszeit als Referenz für die einzelnen zytogenetischen Subgruppen.

Zensiert wurden Patienten für die entsprechenden Berechnungen dann, wenn die definierten Endpunkte (Tod und Entwicklung einer sekundären AML) beim letzten Patientenkontakt im Beobachtungszeitraum nicht eingetreten waren.

Ein $\mathrm{p}$-Wert $<0,05$, das heißt eine Irrtumswahrscheinlichkeit von unter 5\%, wurde als signifikant und ein p-Wert $<0,01$, somit eine Irrtumswahrscheinlichkeit von unter 1\%, als hochsignifikant gewertet. 


\section{Ergebnisse}

\subsection{Beschreibung des Gesamtkollektivs}

\subsubsection{Häufigkeiten der Einzelchromosomenanomalien im Gesamtkollektiv}

Tabelle 7: Absolute und relative Häufigkeitsverteilung der untersuchten Aberrationen

\begin{tabular}{|c|c|c|c|}
\hline $\begin{array}{l}\text { Zytogenetische } \\
\text { Gruppe }\end{array}$ & $\begin{array}{l}\text { Absolute Häufigkeit } \\
\text { (n) }\end{array}$ & $\begin{array}{l}\text { Relative Häufigkeit } \\
\text { aller Datenbankfälle } \\
(n=7012)[\%]\end{array}$ & $\begin{array}{l}\text { Relative Häufigkeit } \\
\text { im untersuchten Kol- } \\
\text { lektiv }(n=382)[\%]\end{array}$ \\
\hline TR1Q & 11 & 0,2 & 2,9 \\
\hline MO3 & 19 & 0,3 & 5,0 \\
\hline MO5 & 8 & 0,1 & 2,1 \\
\hline ANY6 & 8 & 0,1 & 2,1 \\
\hline MO9 & 20 & 0,3 & 5,2 \\
\hline ANY11 & 8 & 0,1 & 2,1 \\
\hline MO12 & 10 & 0,1 & 2,6 \\
\hline MO13Q & 20 & 0,3 & 5,2 \\
\hline MO14 & 10 & 0,1 & 2,6 \\
\hline MO16 & 9 & 0,1 & 2,4 \\
\hline MO18 & 10 & 0,1 & 2,6 \\
\hline ANY20 & 10 & 0,1 & 2,6 \\
\hline TR21 & 17 & 0,2 & 4,5 \\
\hline MOX & 8 & 0,1 & 2,1 \\
\hline DER1_7 & 18 & 0,3 & 4,7 \\
\hline TRB & 67 & 1,0 & 17,5 \\
\hline MAR & 13 & 0,2 & 3,4 \\
\hline OTH & 116 & 1,7 & 30,4 \\
\hline Gesamt & 382 & 5,4 & 100,0 \\
\hline
\end{tabular}




\subsubsection{Geschlechtsverteilung}

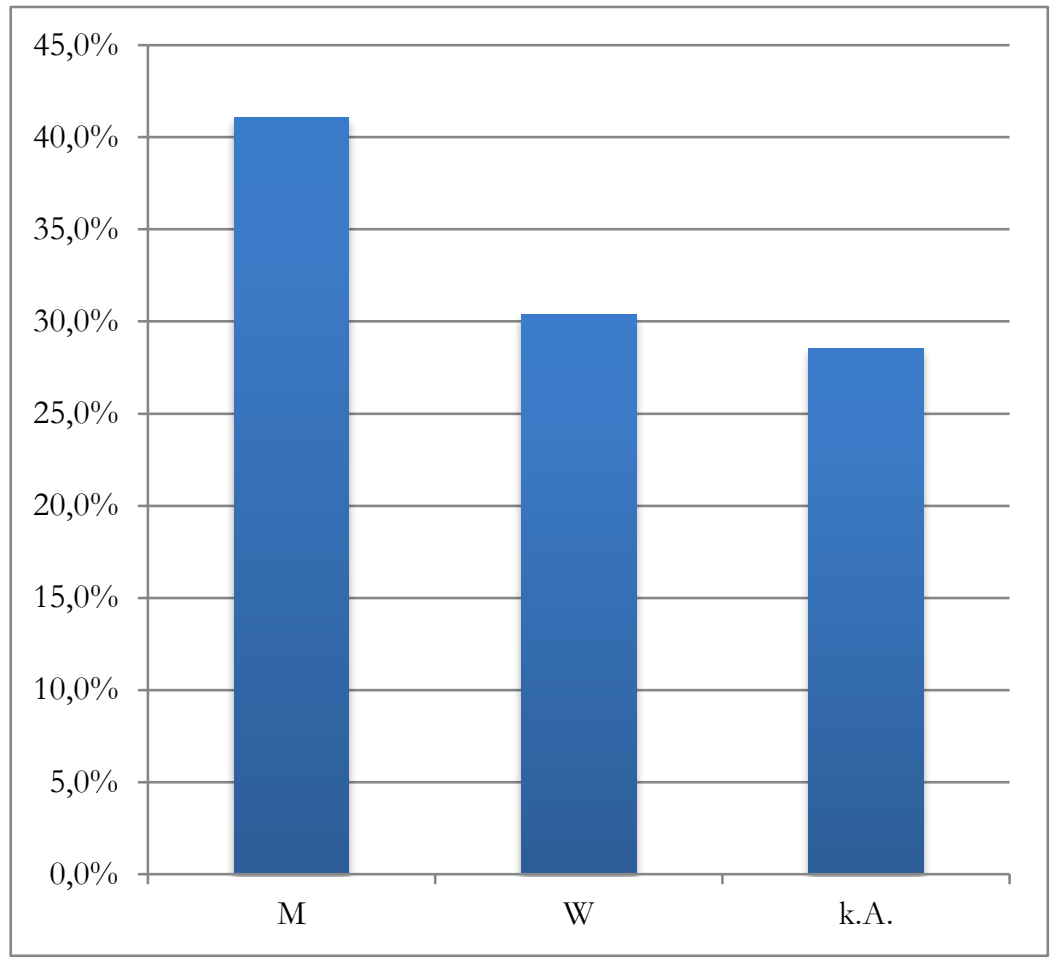

Legende: $M=$ männliche Patienten;

$W=$ weibliche Patienten;

k.A. = Patienten ohne Angabe des Geschlechts

Abbildung 1: Geschlechtsverteilung im Gesamtkollektiv $(n=382)$

\subsubsection{Altersverteilung}

Der Altersmedian bei Diagnose lag bei 67 Jahren, das Minimum bei 20 und das Maximum bei 90 Jahren. 


\subsubsection{MDS-Subtypen nach FAB-Klassifikation im Gesamtkollektiv}

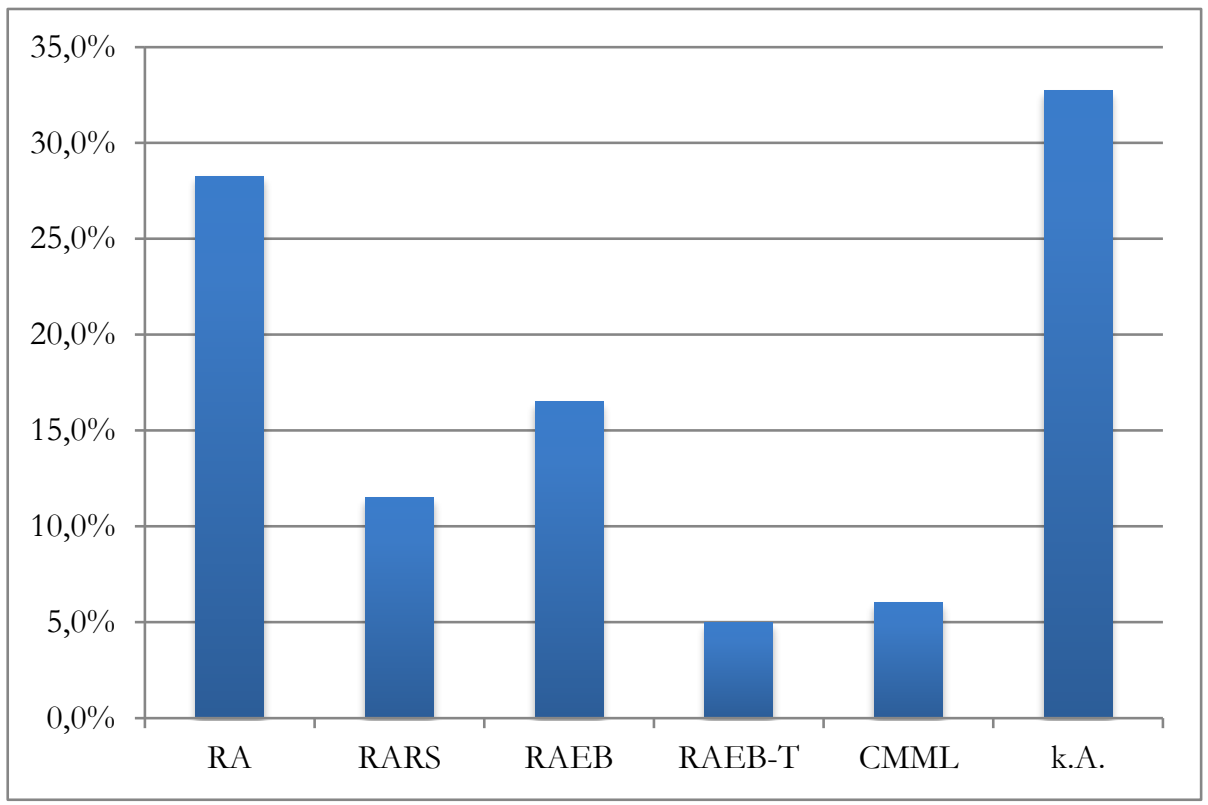

Legende: RA: refraktäre Anämie, RARS: refraktäre Anämie mit Ringsideroblasten; RAEB: refraktäre Anämie mit Blastenexzess; RAEB-T: refraktäre Anämie mit Blastenexzess in Transformation; CMML: chronisch myeloisch-monosytäre Lenkämie; k. A.: Patienten obne Angabe

Abbildung 2: Prozentuale Anteile am Gesamtkollektiv nach FAB-Klassifikation (n=382)

\subsubsection{MDS-Subtypen nach WHO-Klassifikation 2001 und 2008 im Gesamtkollektiv}

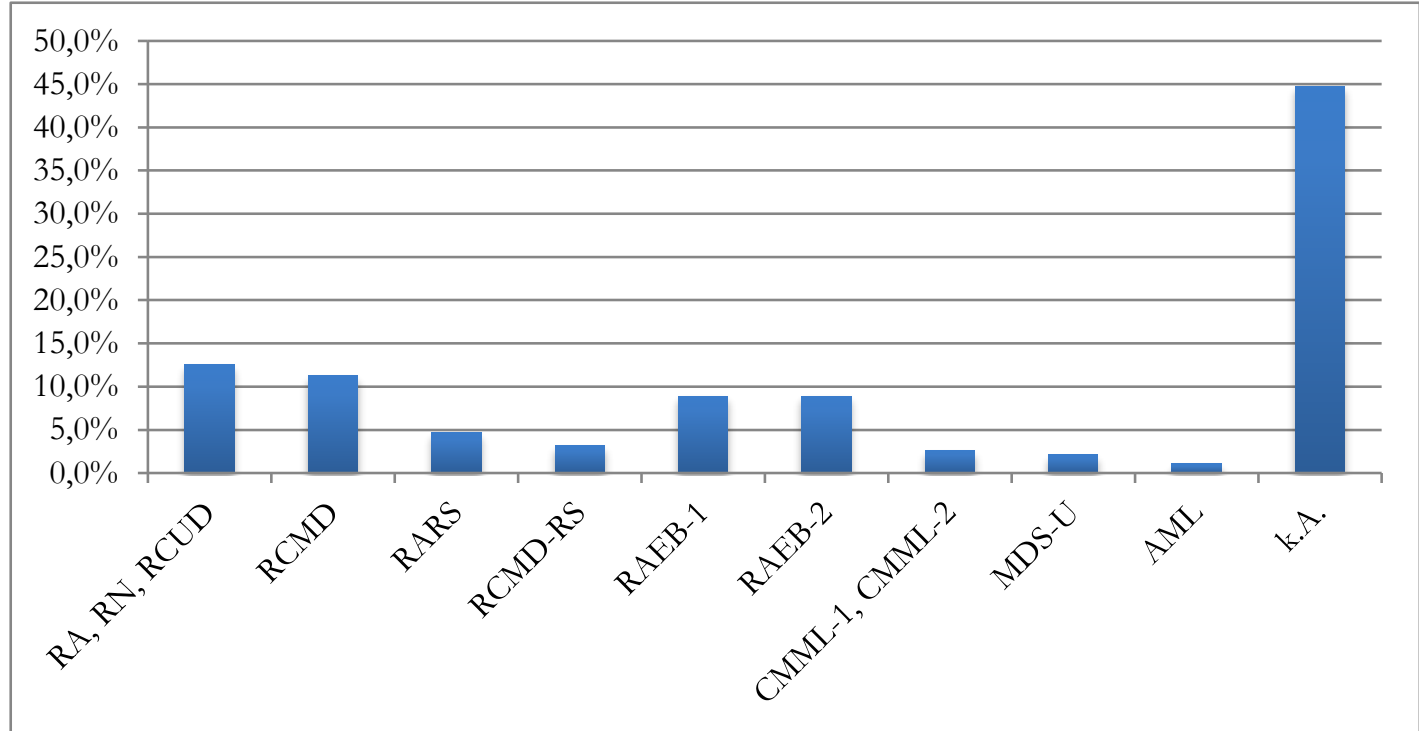

Legende: RA(-RS): refraktäre Anämie (-mit Ringsideroblasten); RN: refraktäre Neutropenie; RCUD: refraktäre Zytopenie mit unilineärer Dysplasie; RCMD(-RS): refraktäre Zytopenie mit multilineärer Dysplasie (-mit Ringsideroblasten); RAEB (-1/-2): refraktäre Anämie mit Blastenexzess (5-9\%/10-19\%); CMML (-1/-2): chronisch myeloischmonocytäre Leukämie (5-9\%/10-19\%); MDS-U: Myelodysplastisches Syndrom, unklassifizierbar; AML: akute myeloische Lenkämie; k.A.: Patienten obne Angabe

Abbildung 3: Prozentuale Anteile am Gesamtkollektiv nach WHO-Klassifikation (n=382) 
Da in der WHO-Klassifikation von 2001 die Grenze zur Definition einer AML von 30\% auf 20\% Blasten im Knochenmark gesenkt wurde, enthält die Darstellung einige wenige Fälle, die nun als AML klassifiziert wurden. Die Fälle für Refraktäre Anämie, Refraktäre Neutropenie und Refraktäre Zytopenie mit unilineärer Dysplasie entsprechen sich in den Klassifikationen von 2001 und 2008 und wurden deshalb zusammengefasst. Die Kategorie RCMDRS ist in 2008 nicht mehr enthalten. Diese Fälle wurden folglich nach der Klassifikation von 2001 eingeteilt.

\subsection{6 Überlebenszeit (ÜLZ) für das Gesamtkollektiv}

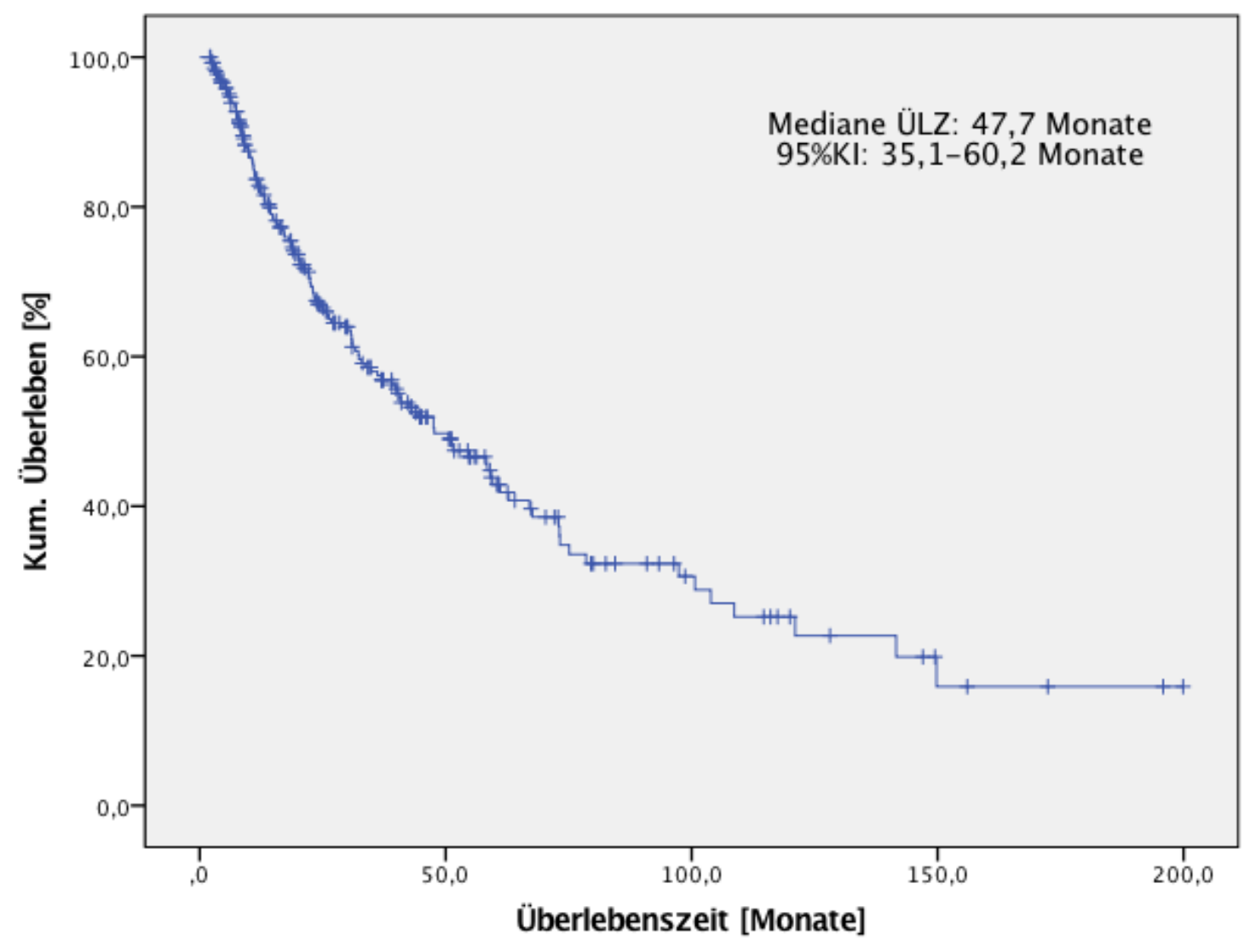

Abbildung 4: Kaplan-Meier-Überlebensfunktion für alle Patienten $(n=272)$

\subsubsection{AML-freies Überleben (AFS) im Gesamtkollektiv}

Bei insgesamt 238 verarbeiteten Fällen traten 40 sekundäre AML auf. 198 Patienten waren zensiert. Der Mittelwert für das AML-freie Überleben liegt bei 145,9 Monaten, mit einem 95\% Konfidenzintervall (KI) von 128,7-163,1 Monaten. In der Analyse des AML-freien Überlebens nach FAB- und WHO-Subgruppen zeigt sich bei insgesamt geringer Fallzahl ein kürzeres AML-freies Überleben in den Subgruppen mit erhöhtem Blastenanteil (RAEB, RAEB-T, bzw. RAEB-1, RAEB-2). 


\subsection{8 Überlebenskurve nach Geschlecht}

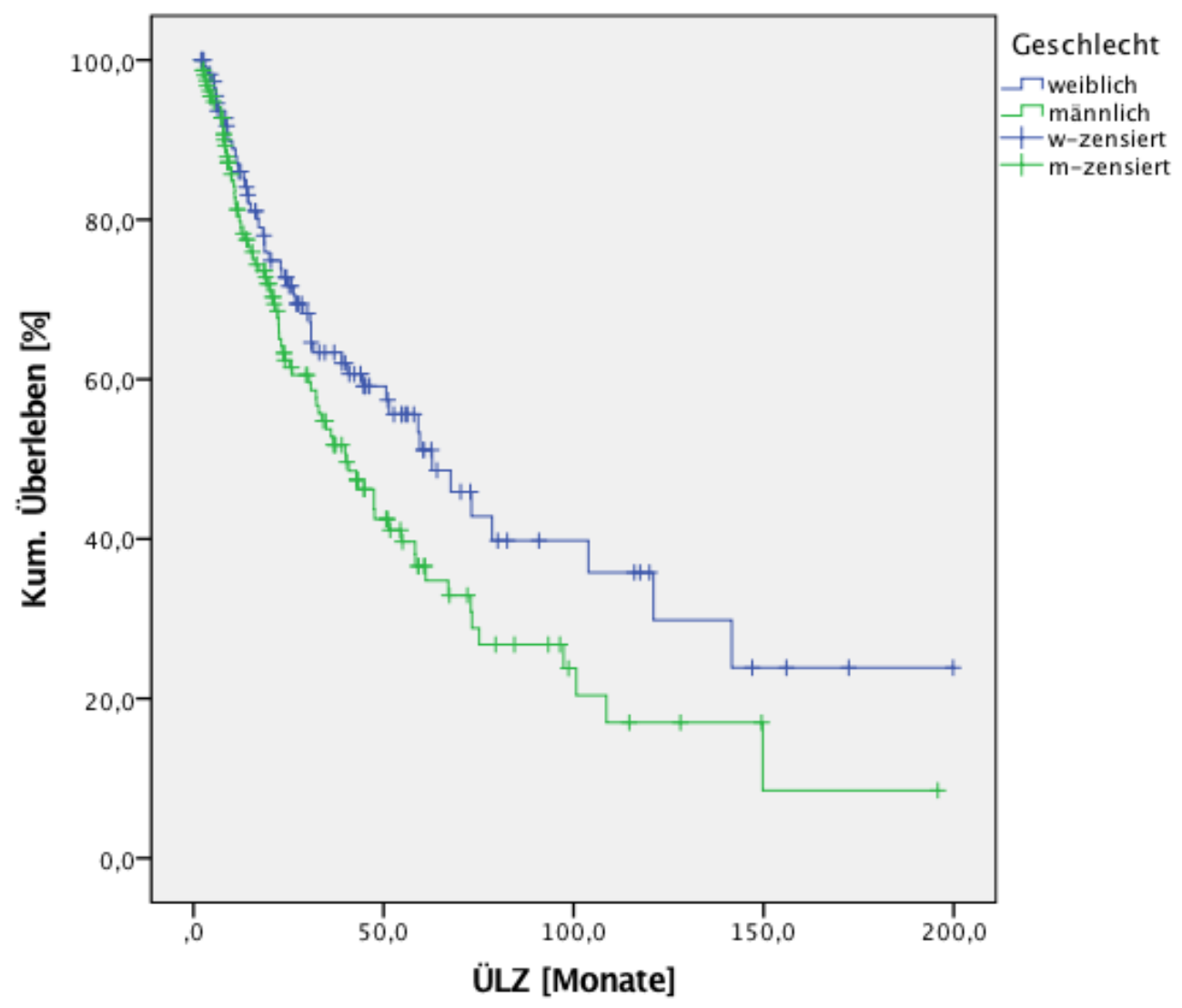

Abbildung 5: Kaplan-Meier-Überlebensfunktion nach Geschlecht $(n=272)$

Es zeigt sich für die Frauen eine mediane Überlebenszeit (ÜLZ) von 62,8 Monaten (95\% KI: 42,6-83,1 Monate) und für die Männer eine mediane Überlebenszeit von 40,1 Monaten (95\% KI: 29,0-51,1 Monate).

\subsection{Univariate Analyse nach zytogenetischen Subgruppen}

Zwischen den einzelnen Gruppen konnten signifikante Unterschiede hinsichtlich der stetigen Variablen (Alter, Hb, ANC, Monozyten, Metaphasen, Thrombozyten, Leukozyten, Blasten im peripheren Blut und im Knochenmark) mit Hilfe des Kruksal-Walis-Anova-Tests ausgeschlossen werden.

Der Log-Rank-Test ergab für die Überlebenszeit einen signifikanten negativen Einfluss $(\mathrm{p}=0,033)$ des männlichen Geschlechts.

Bei der Untersuchung der AML-freien Überlebenszeit hatte das Geschlecht keinen signifikanten Einfluss auf die Entwicklung einer sekundären AML $(p=0,168)$. 
3.2.1 Überlebenszeit in der Subgruppe von Patienten mit Trisomie (1q) (TR1Q)

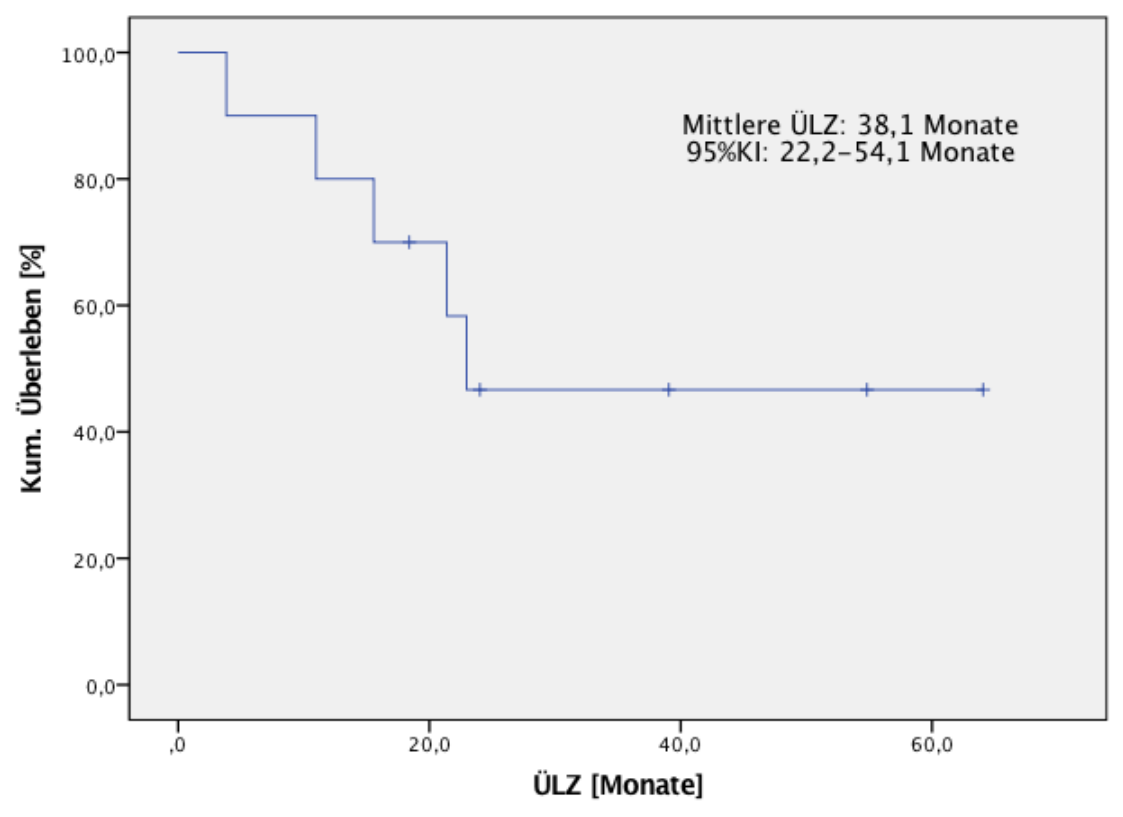

Abbildung 6: Kaplan-Meier-Überlebensfunktion für TR1Q ( $n=10)$

3.2.2 AML-freie Überlebenszeit in der Subgruppe von Patienten mit Trisomie (1q) (TR1Q)

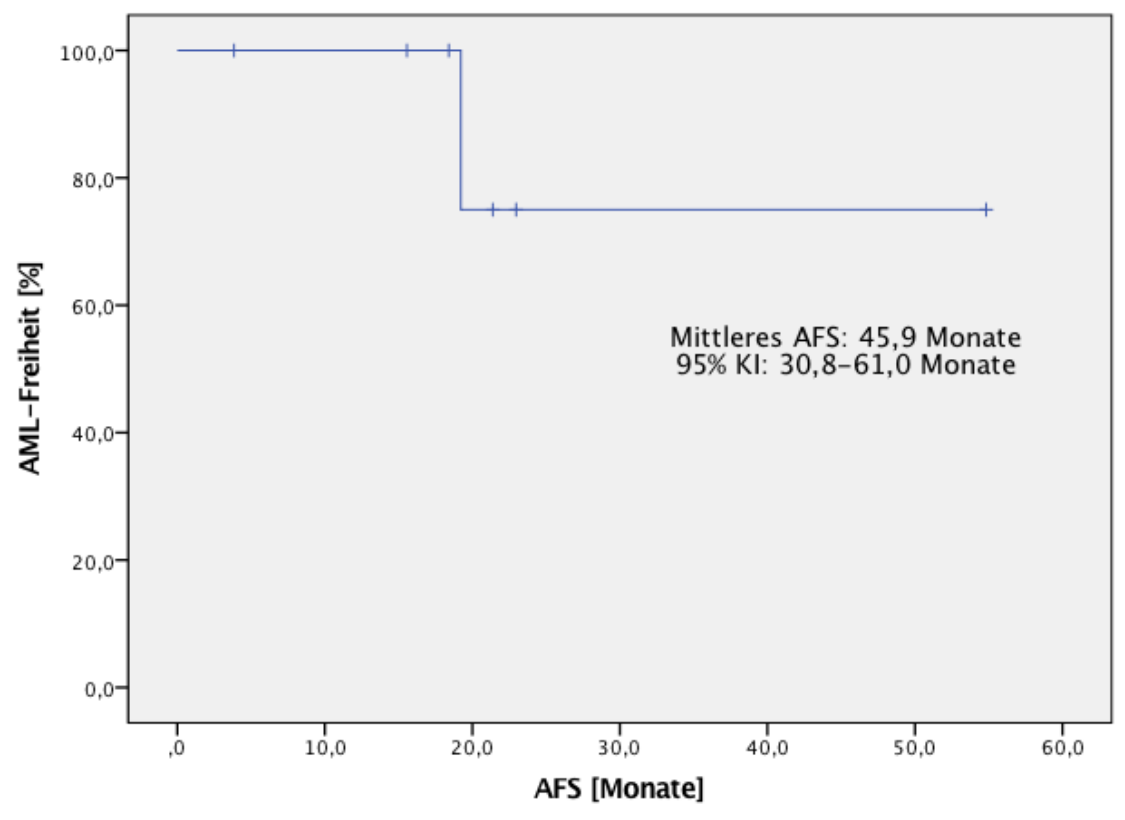

Abbildung 7: Kaplan-Meier-Funktion für AFS TR1Q (n=7) 


\subsection{3 Überlebenszeit in der Subgruppe von Patienten mit Monosomie 3 (MO3)}

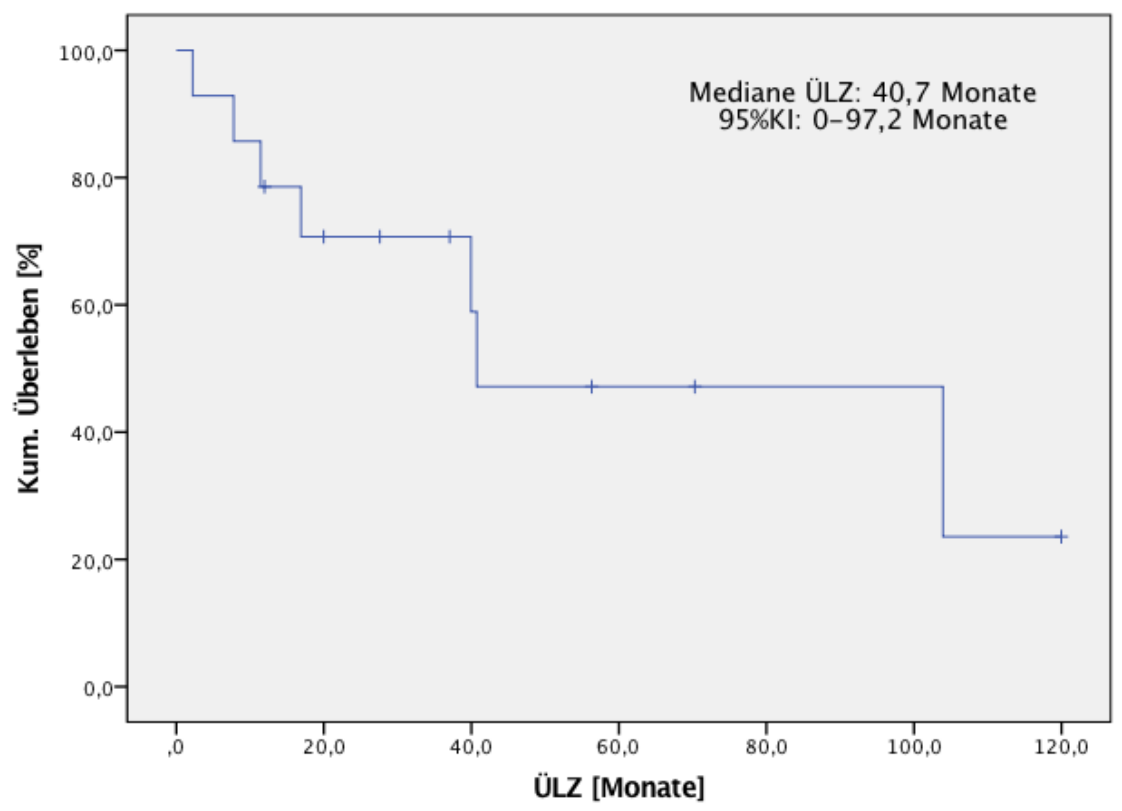

Abbildung 8: Kaplan-Meier-Überlebensfunktion für MO3 (n=14)

3.2.4 AML-freie Überlebenszeit in der Subgruppe von Patienten mit Monosomie 3 (MO3)

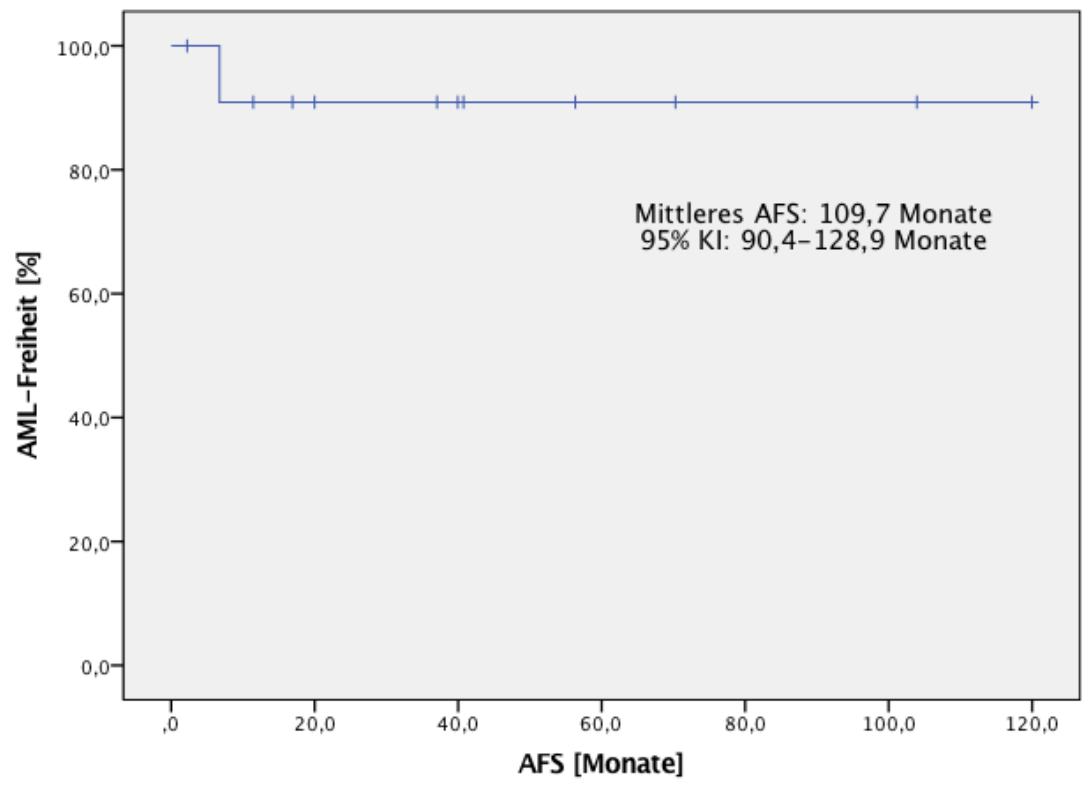

Abbildung 9: Kaplan-Meier-Funktion für AFS MO3 (n=12) 


\subsection{5 Überlebenszeit in der Subgruppe von Patienten mit Monosomie 5 (MO5)}

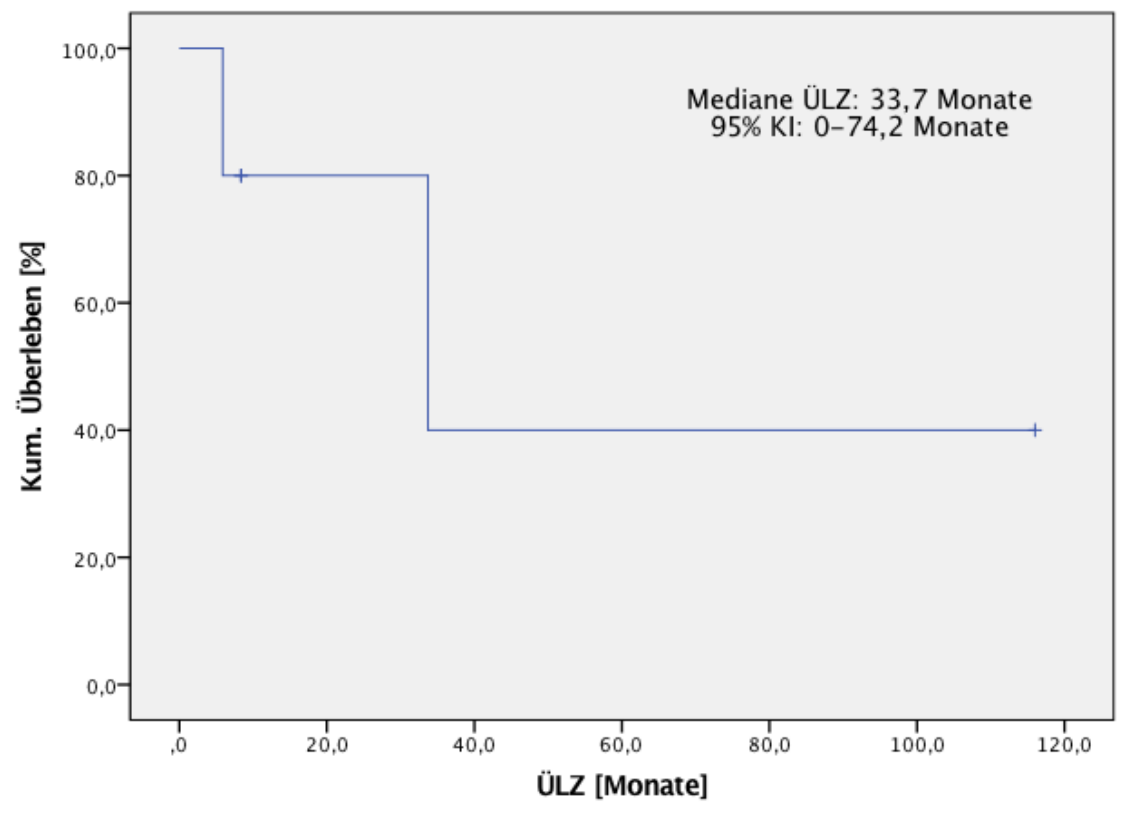

Abbildung 10: Kaplan-Meier-Überlebensfunktion für MO5 (n=5)

3.2.6 AML-freie Überlebenszeit in der Subgruppe von Patienten mit Monosomie 5 (MO5)

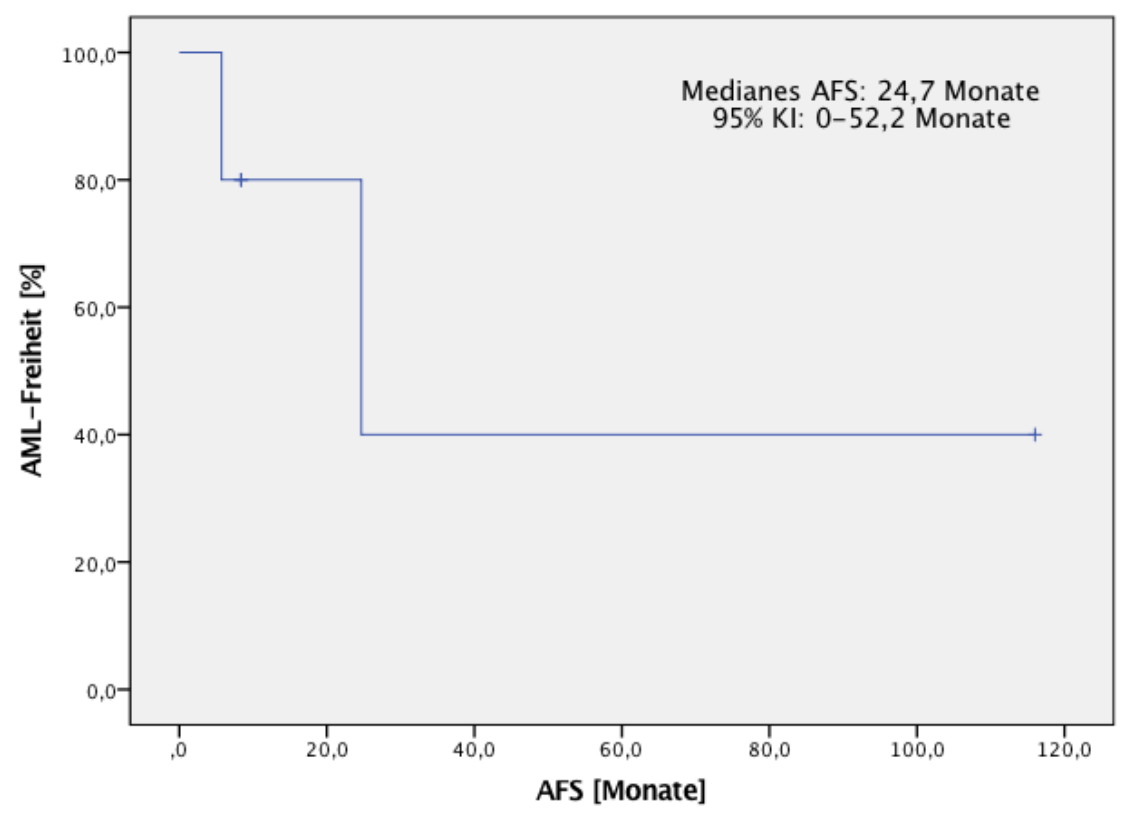

Abbildung 11: Kaplan-Meier-Funktion für AFS MO5 (n=5) 


\subsection{7 Überlebenszeit in der Subgruppe von Patienten mit Veränderungen am}

Chromosom 6 (ANY6)

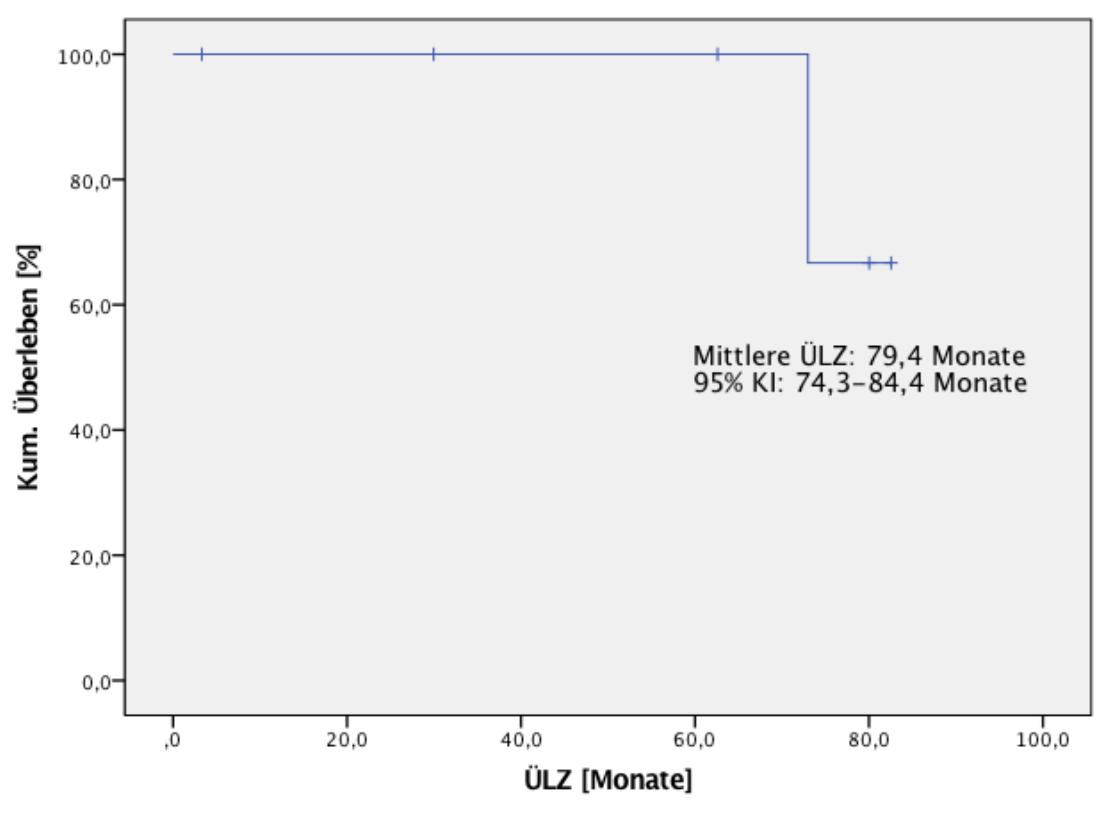

Abbildung 12: Kaplan-Meier-Überlebensfunktion für ANY6 (n=6)

\subsubsection{AML-freie Überlebenszeit in der Subgruppe von Patienten mit} Veränderungen am Chromosom 6 (ANY6)

In der Subgruppe der Patienten mit Veränderungen am Chromosom $6(n=5)$ traten im Beobachtungszeitraum keine sekundären AML auf. Daher wurde keine Kaplan-Meier-Funktion für die AML-freie Überlebenszeit erstellt. 


\subsection{9 Überlebenszeit in der Subgruppe von Patienten mit Monosomie 9 (MO9)}

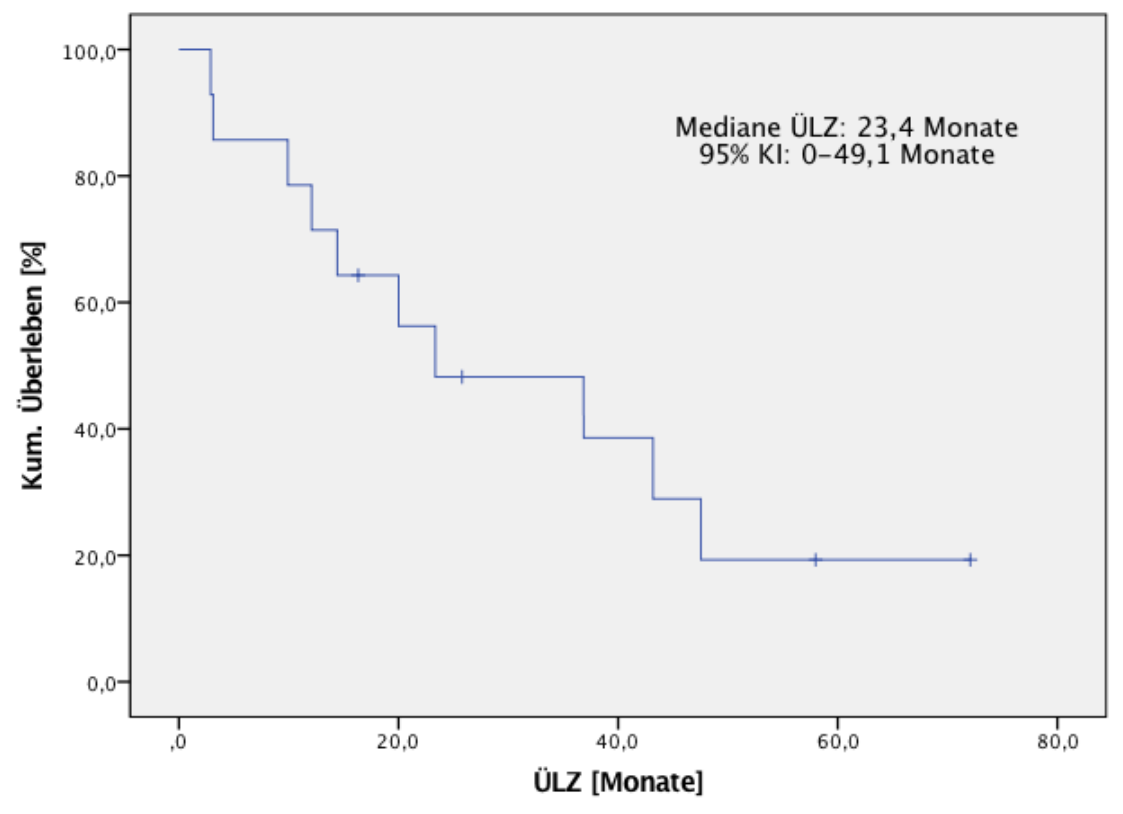

Abbildung 13: Kaplan-Meier-Überlebensfunktion für MO9 (n=14)

3.2.10 AML-freie Überlebenszeit in der Subgruppe von Patienten mit Monosomie 9 (MO9)

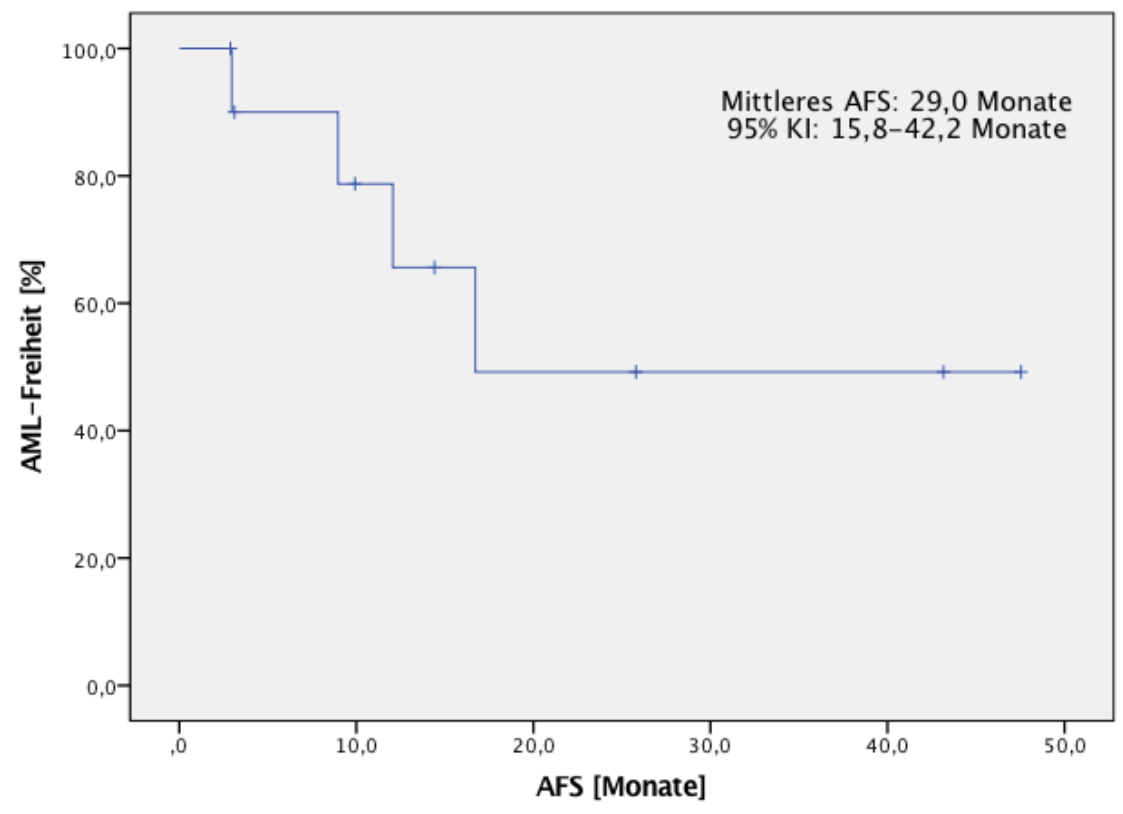

Abbildung 14: Kaplan-Meier-Funktion für AFS MO9 (n=11) 
3.2.11 Überlebenszeit in der Subgruppe von Patienten mit Veränderungen am Chromosom 11 (ANY11)

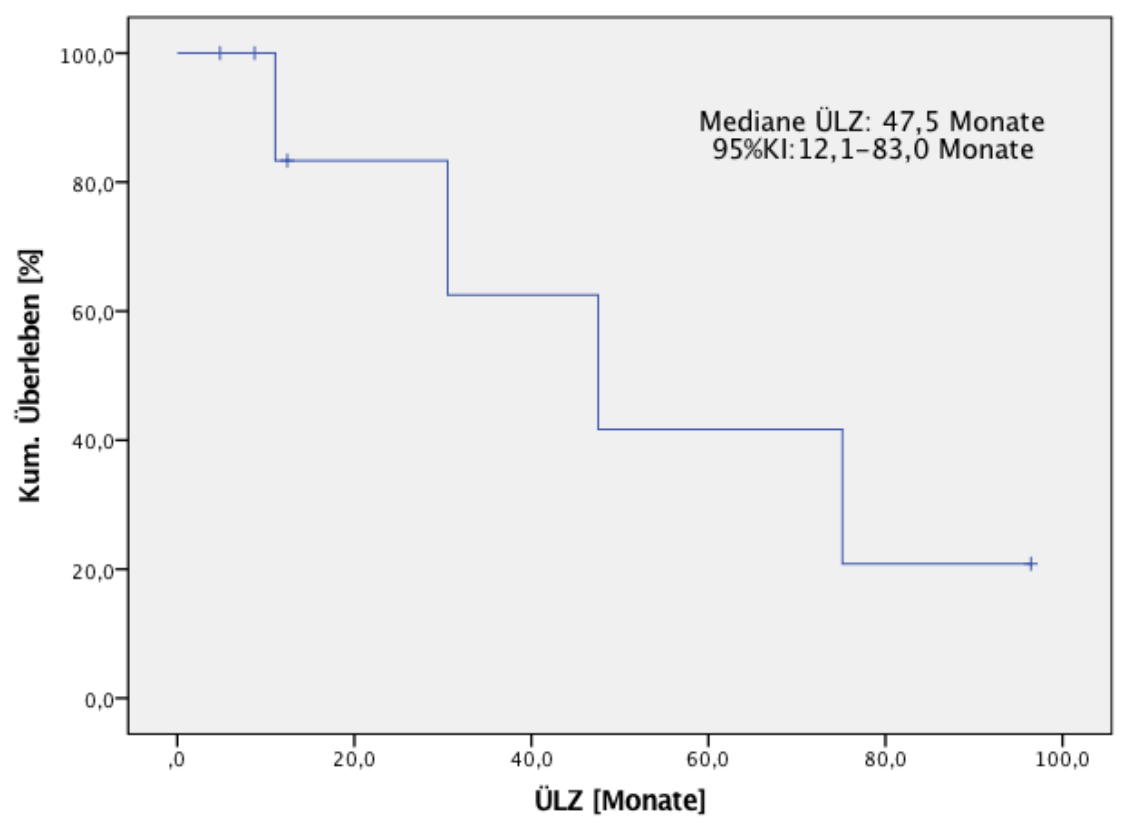

Abbildung 15: Kaplan-Meier-Überlebensfunktion für ANY11 (n=8)

3.2.12 AML-freie Überlebenszeit in der Subgruppe von Patienten mit Veränderungen am Chromosom 11 (ANY11)

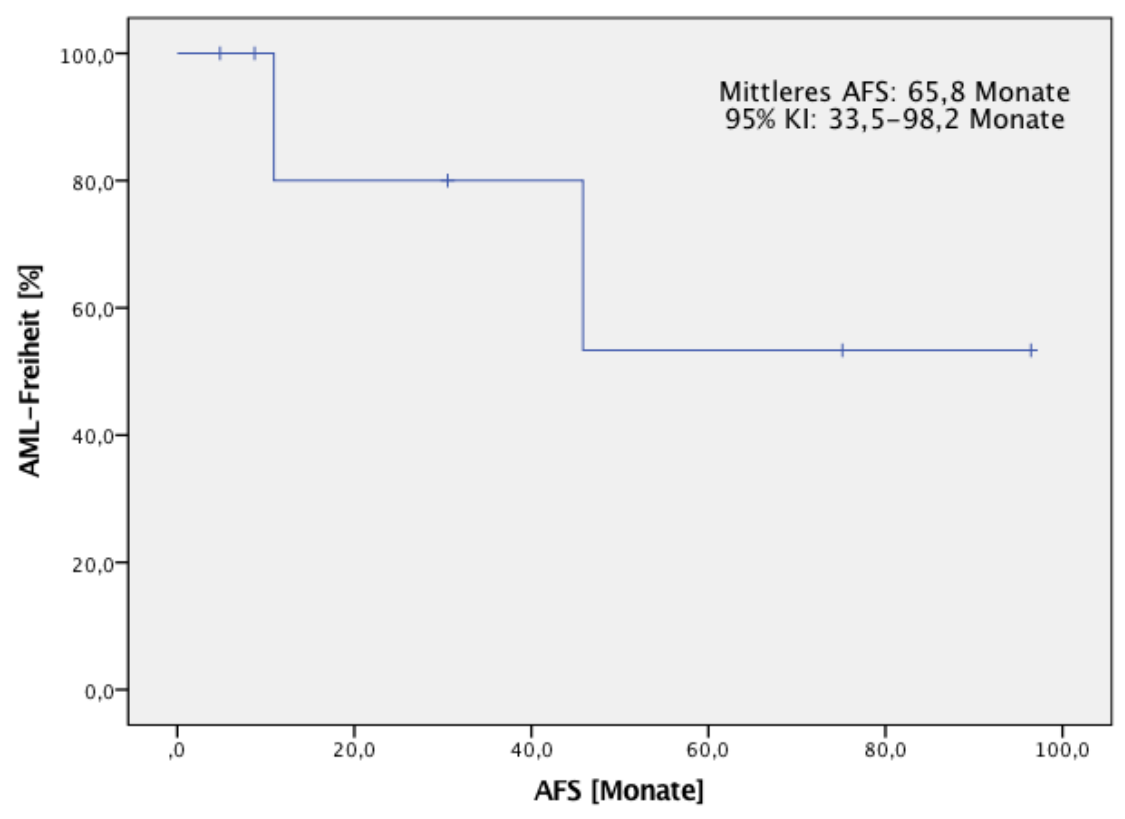

Abbildung 16: Kaplan-Meier-Funktion für AFS ANY11 (n=7) 
3.2.13 Überlebenszeit in der Subgruppe von Patienten mit Monosomie 12 (MO12)

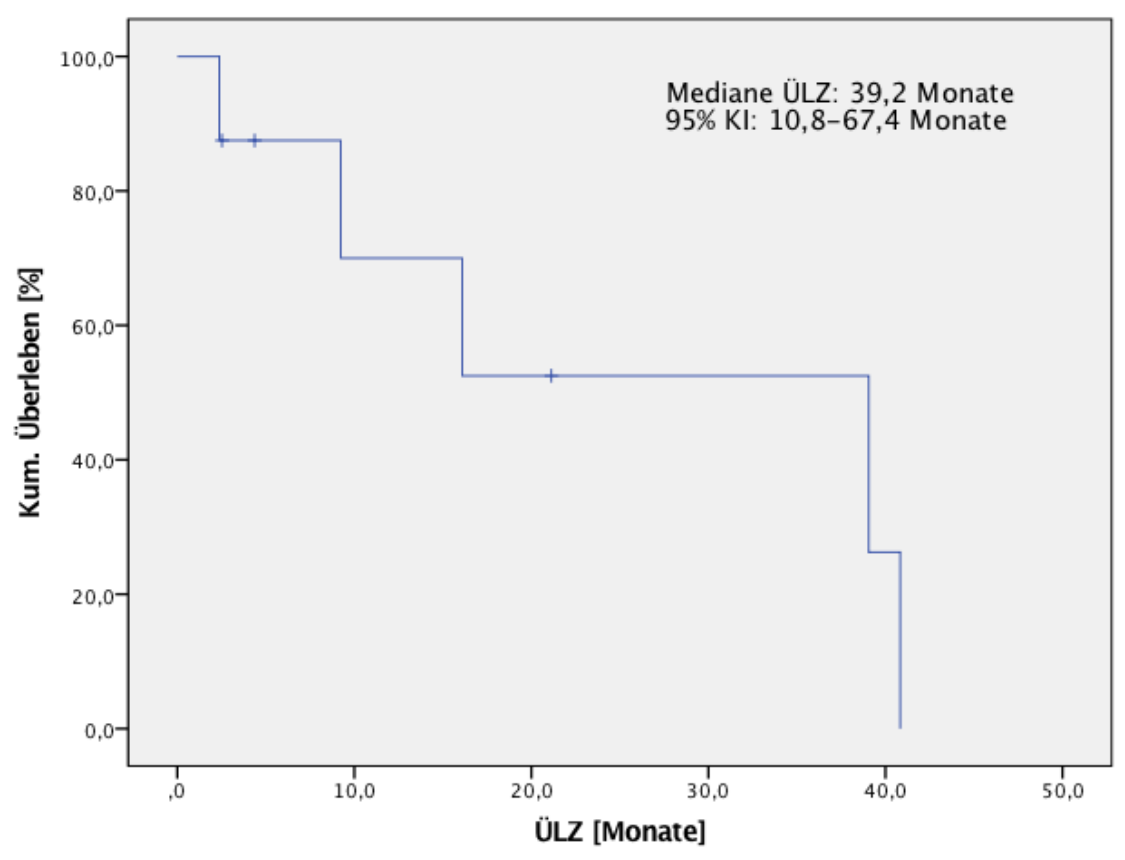

Abbildung 17: Kaplan-Meier-Überlebensfunktion für MO12 (n=8)

3.2.14 AML-freie Überlebenszeit in der Subgruppe von Patienten mit Monosomie 12 (MO12)

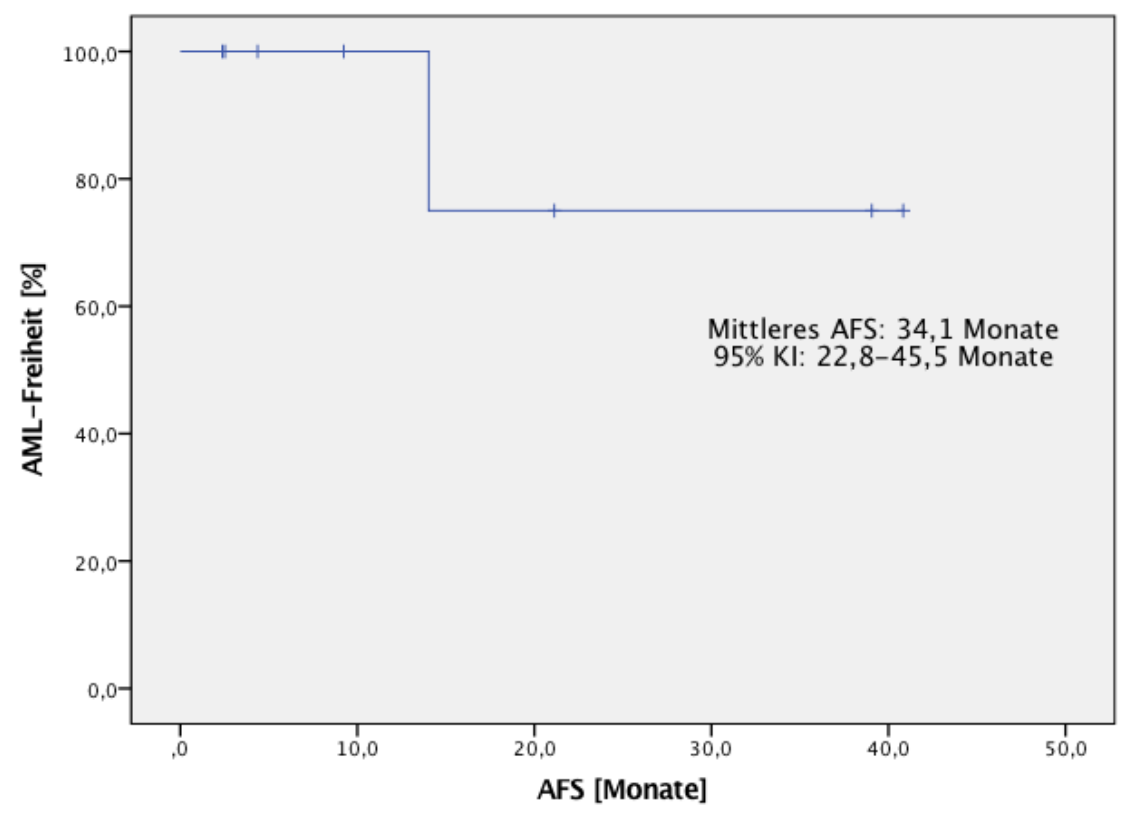

Abbildung 18: Kaplan-Meier-Funktion für AFS MO12 (n=8) 


\subsubsection{5 Überlebenszeit in der Subgruppe von Patienten mit Monosomie (13q) (MO13Q)}

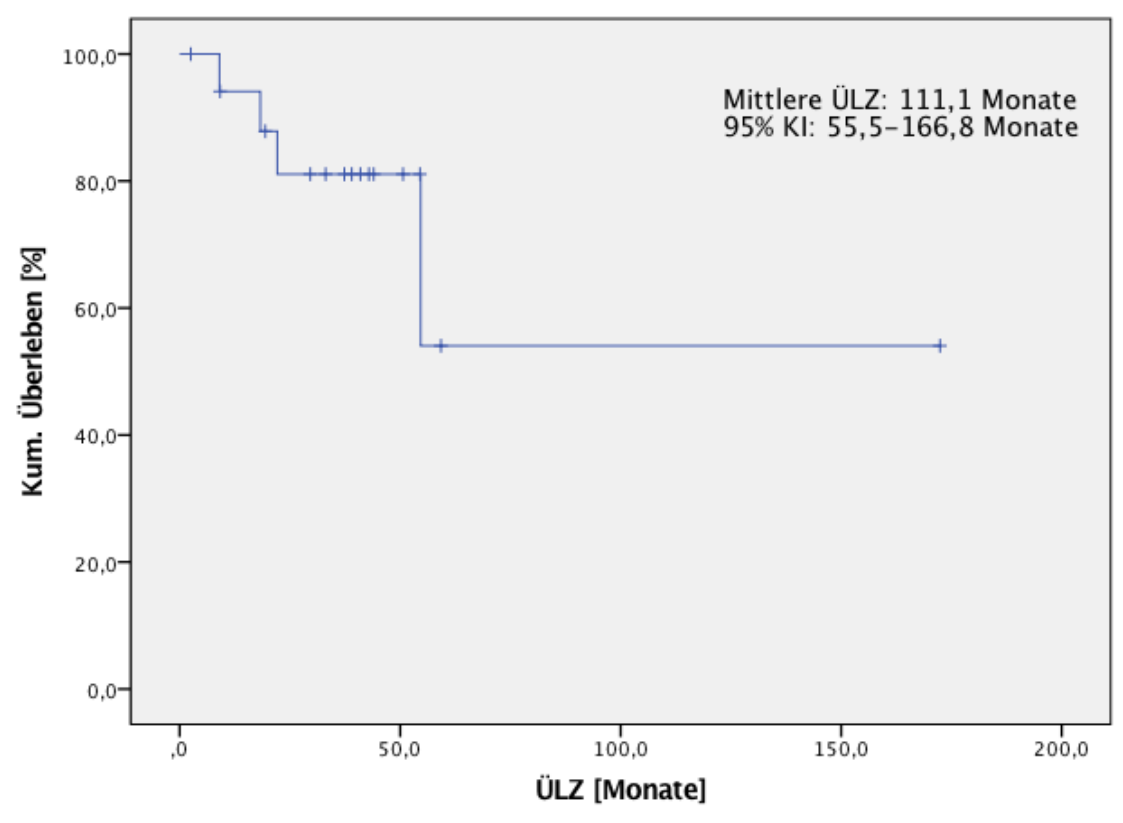

Abbildung 19: Kaplan-Meier-Überlebensfunktion für MO13Q (n=18)

\subsubsection{AML-freie Überlebenszeit in der Subgruppe von Patienten mit Monosomie (13q) (MO13Q)}

In der Subgruppe der Patienten mit Monosomie(13q) ( $n=14)$ traten im Beobachtungszeitraum keine sekundären AML auf. Daher wurde keine Kaplan-Meier-Funktion für die AMLfreie Überlebenszeit erstellt. 


\subsubsection{7 Überlebenszeit in der Subgruppe von Patienten mit Monosomie 14 (MO14)}

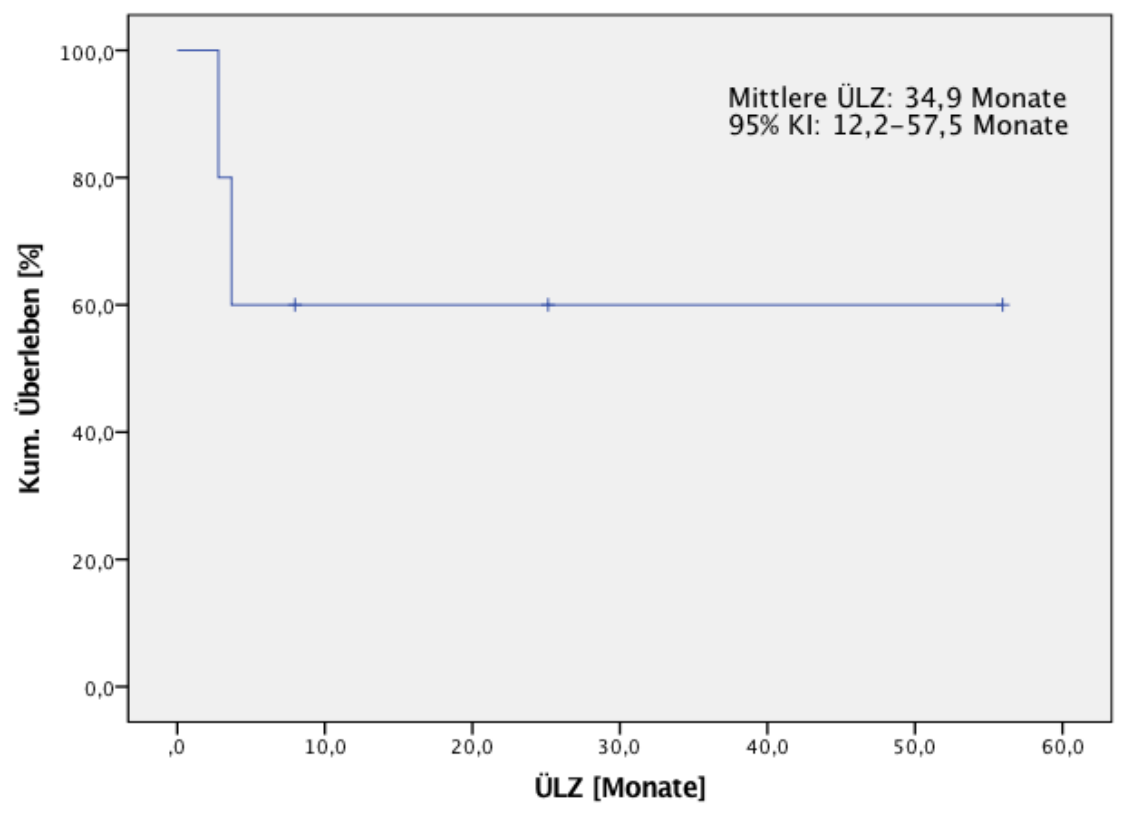

Abbildung 20: Kaplan-Meier-Überlebensfunktion für MO14 (n=5)

\subsubsection{AML-freie Überlebenszeit in der Subgruppe von Patienten mit} Monosomie 14 (MO14)

In der Subgruppe der Patienten mit Monosomie $14(n=5)$ traten im Beobachtungszeitraum keine Fälle einer sekundären AML auf. Daher wurde keine Kaplan-Meier-Funktion für die AML-freie Überlebenszeit erstellt.

\subsubsection{9 Überlebenszeit in der Subgruppe von Patienten mit Monosomie 16 (MO16)}

In der Gruppe der Patienten mit Monosomie $16(\mathrm{n}=8)$ traten im Beobachtungszeitraum keine Todesfälle auf. Alle Fälle waren zensiert. Daher wurde keine Kaplan-Meier-Überlebensfunktion erstellt.

\subsubsection{AML-freie Überlebenszeit in der Subgruppe von Patienten mit Monosomie 16 (MO16)}

In der Subgruppe von Patienten mit Monosomie $16(n=7)$ traten im Beobachtungszeitraum keine Fälle einer sekundären AML auf. Daher wurde keine Kaplan-Meier-Funktion für die AML-freie Überlebenszeit dieser Gruppe erstellt. 
3.2.21 Überlebenszeit in der Subgruppe von Patienten mit Monosomie 18 (MO18)

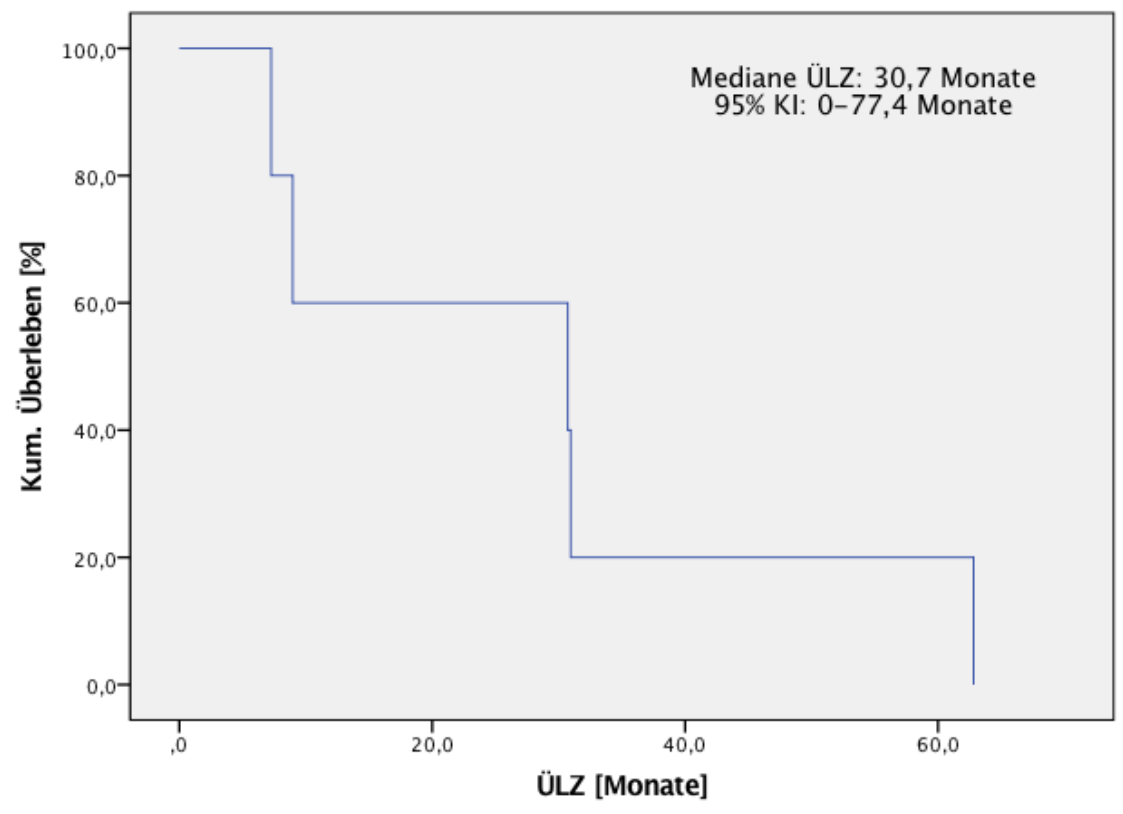

Abbildung 21: Kaplan-Meier-Überlebensfunktion für MO18 (n=5)

3.2.22 AML-freie Überlebenszeit in der Subgruppe von Patienten mit Monosomie 18 (MO18)

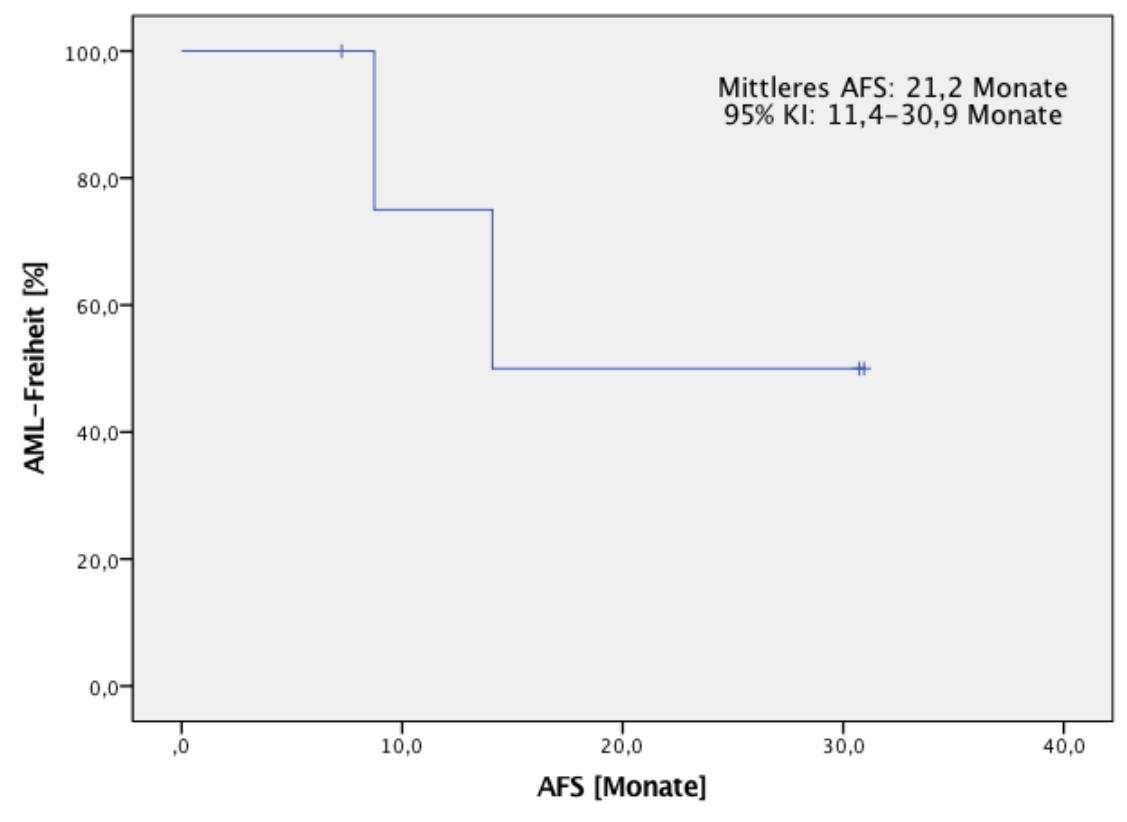

Abbildung 22: Kaplan-Meier-Funktion für AFS MO18 (n=5) 
3.2.23 Überlebenszeit in der Subgruppe von Patienten mit Veränderungen am Chromosom 20 (ANY20)

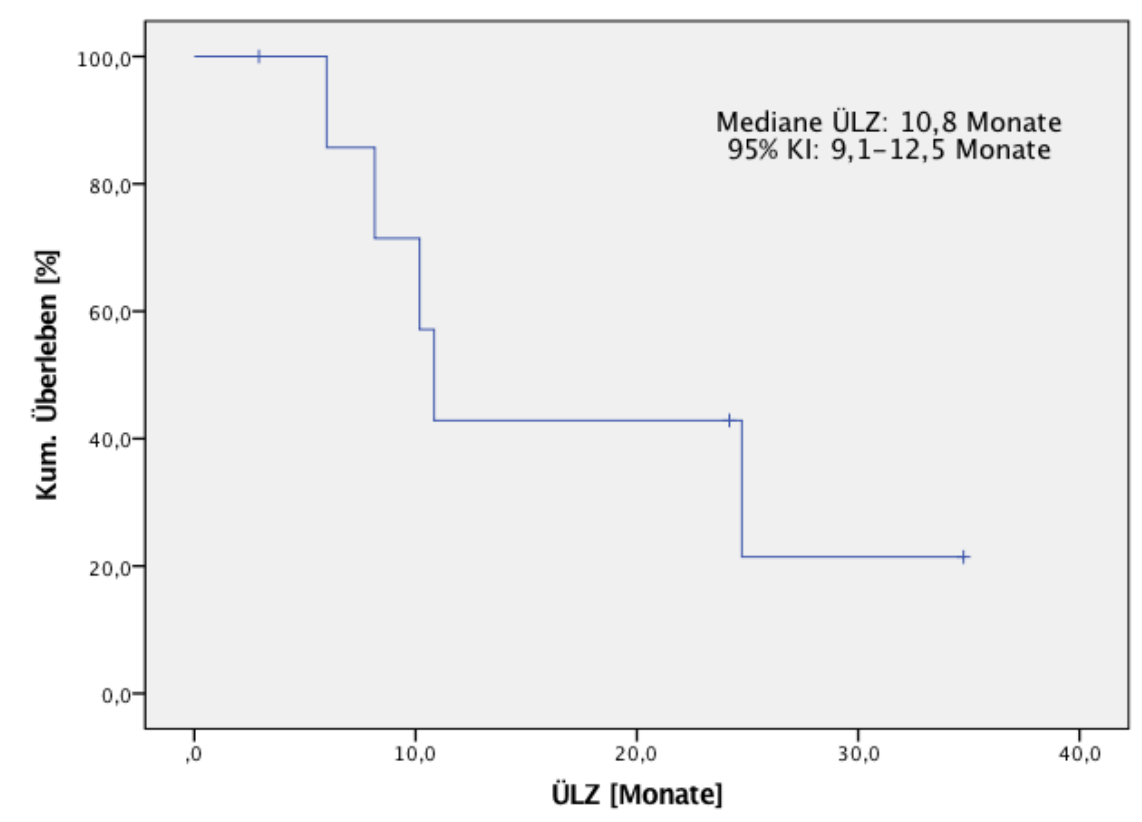

Abbildung 23: Kaplan-Meier-Überlebensfunktion für ANY20 (n=8)

3.2.24 AML-freie Überlebenszeit in der Subgruppe von Patienten mit Veränderungen am Chromosom 20 (ANY20)

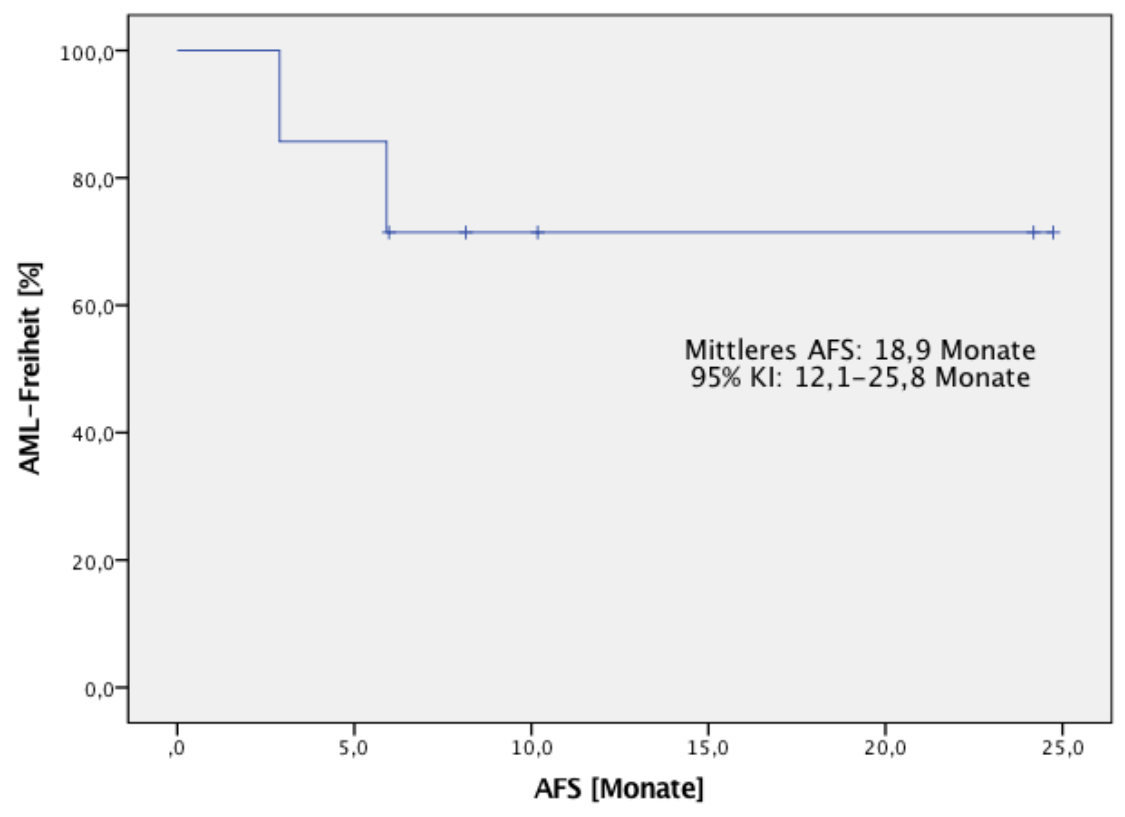

Abbildung 24: Kaplan-Meier-Funktion für AFS ANY20 (n=7) 
3.2.25 Überlebenszeit in der Subgruppe von Patienten mit Trisomie 21 (TR21)

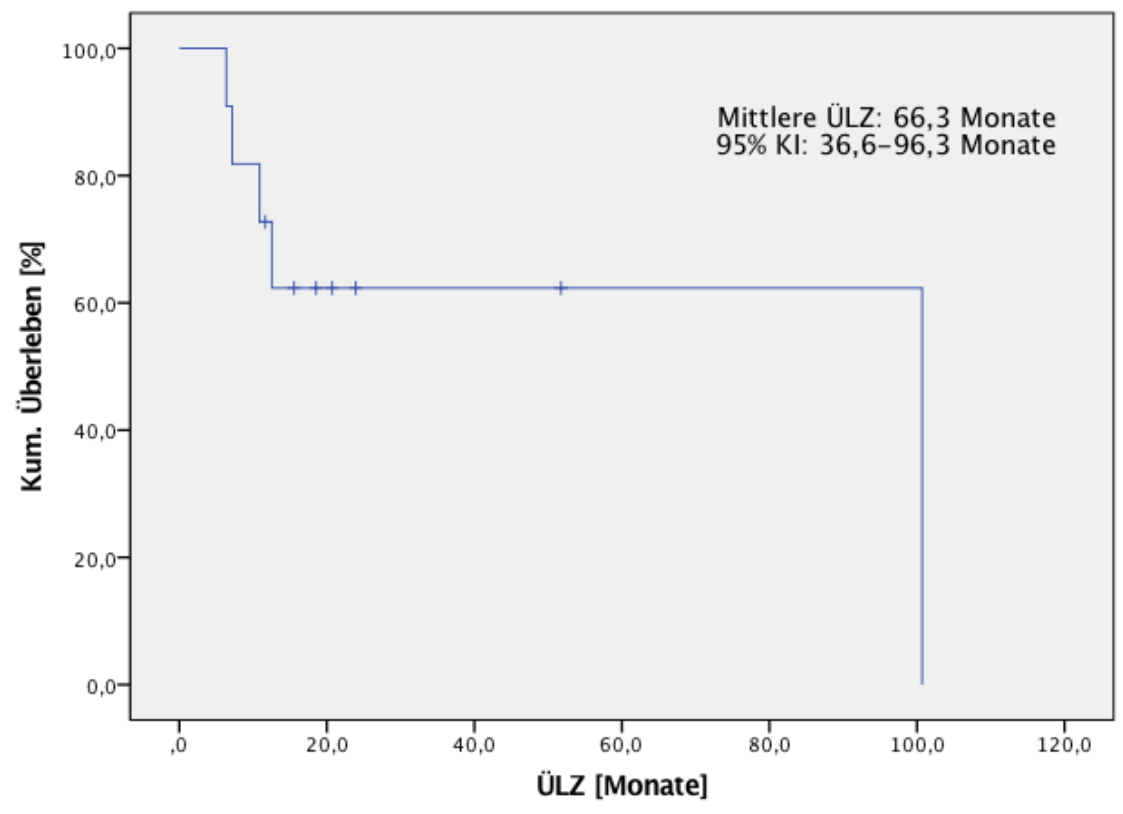

Abbildung 25: Kaplan-Meier-Überlebensfunktion für TR21 (n=11)

3.2.26 AML-freie Überlebenszeit in der Subgruppe von Patienten mit Trisomie 21 (TR21)

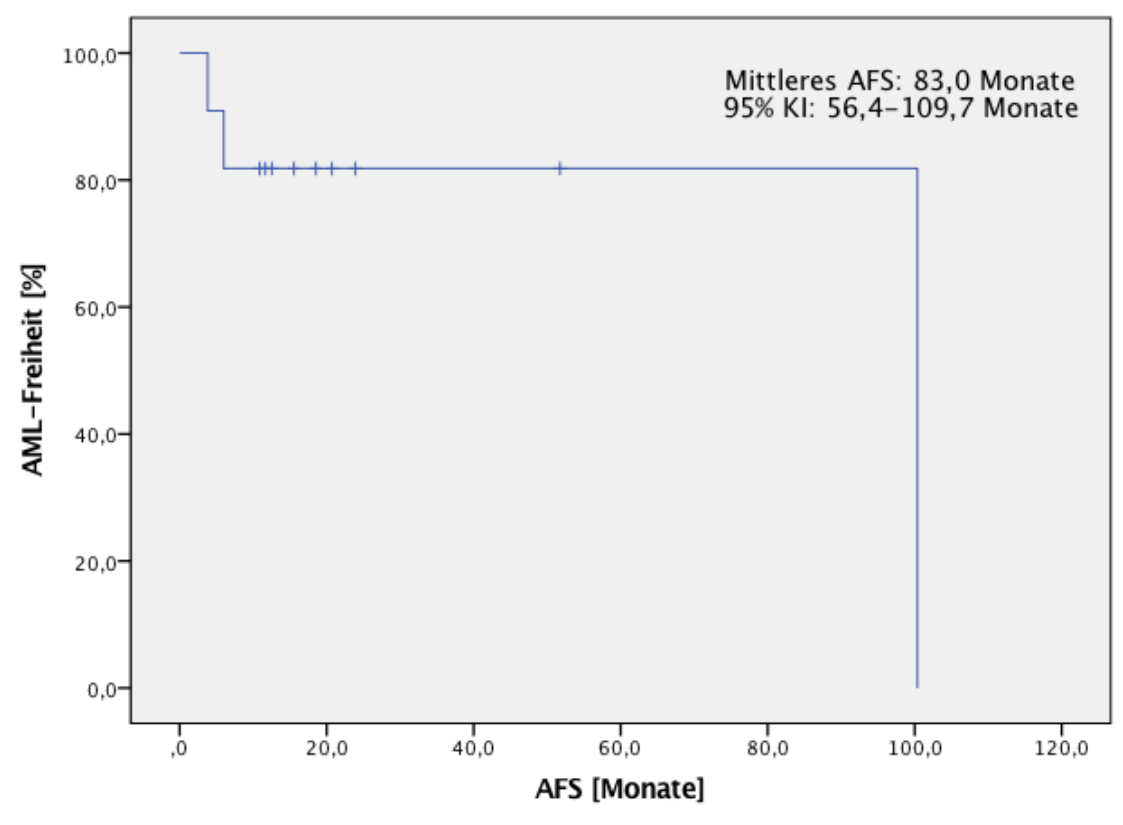

Abbildung 26: Kaplan-Meier-Funktion für AFS TR21 (n=11) 
3.2.27 Überlebenszeit in der Subgruppe von Patienten mit Monosomie X (MOX)

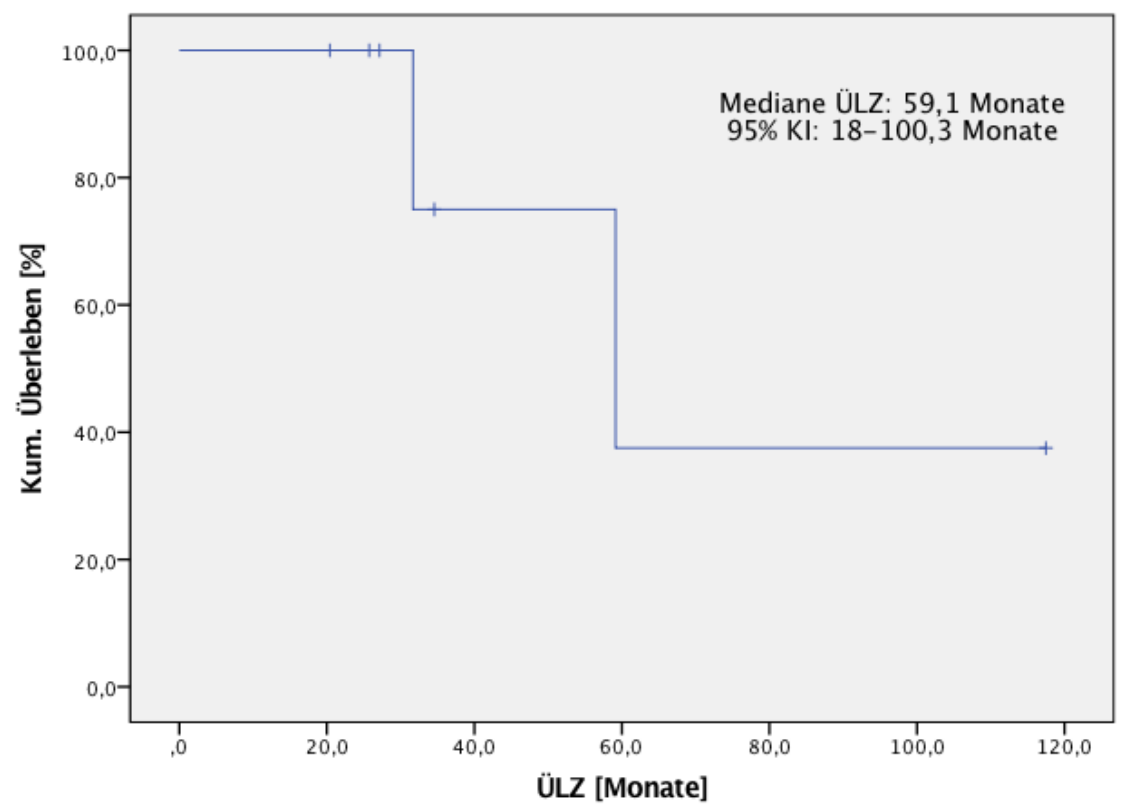

Abbildung 27: Kaplan-Meier-Überlebensfunktion für MOX ( $\mathrm{n}=7)$

3.2.28 AML-freie Überlebenszeit in der Subgruppe von Patienten mit Monosomie X (MOX)

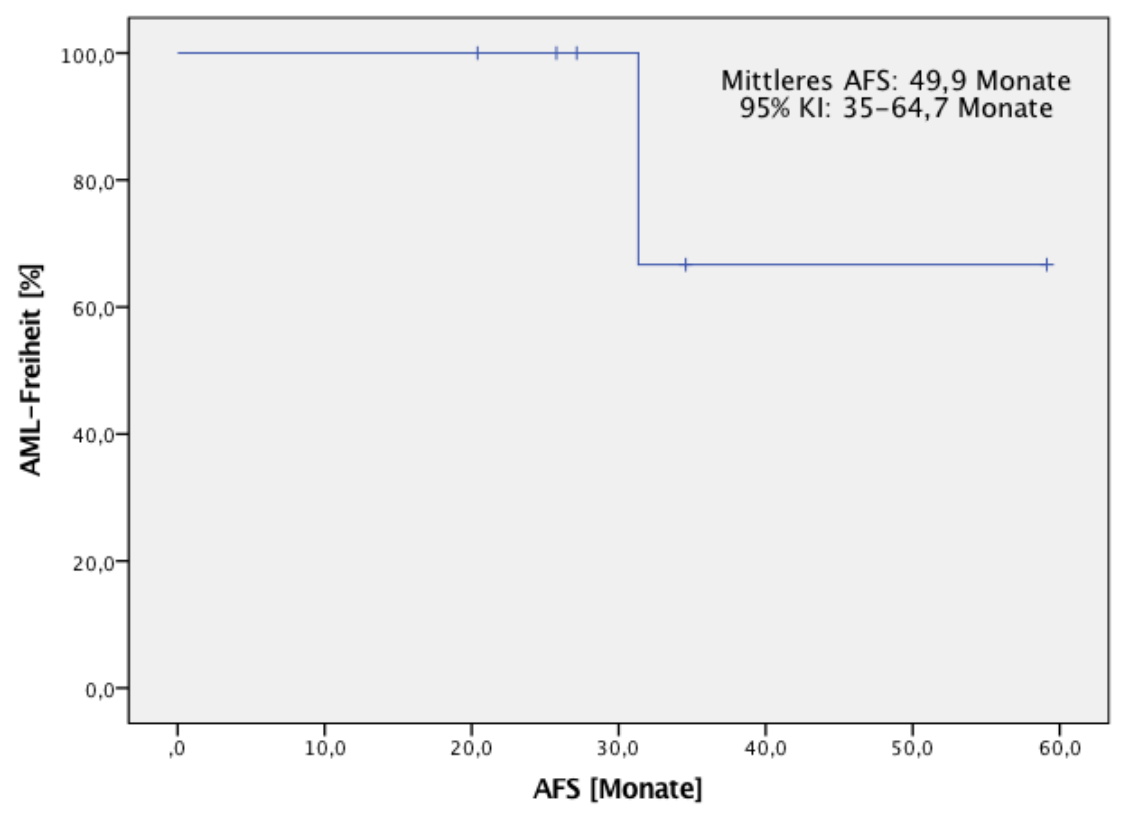

Abbildung 28: Kaplan-Meier-Funktion für AFS MOX ( $n=6)$ 


\subsubsection{9 Überlebenszeit in der Subgruppe von Patienten mit derivativem} Chromosom (1;7) DER(1;7)

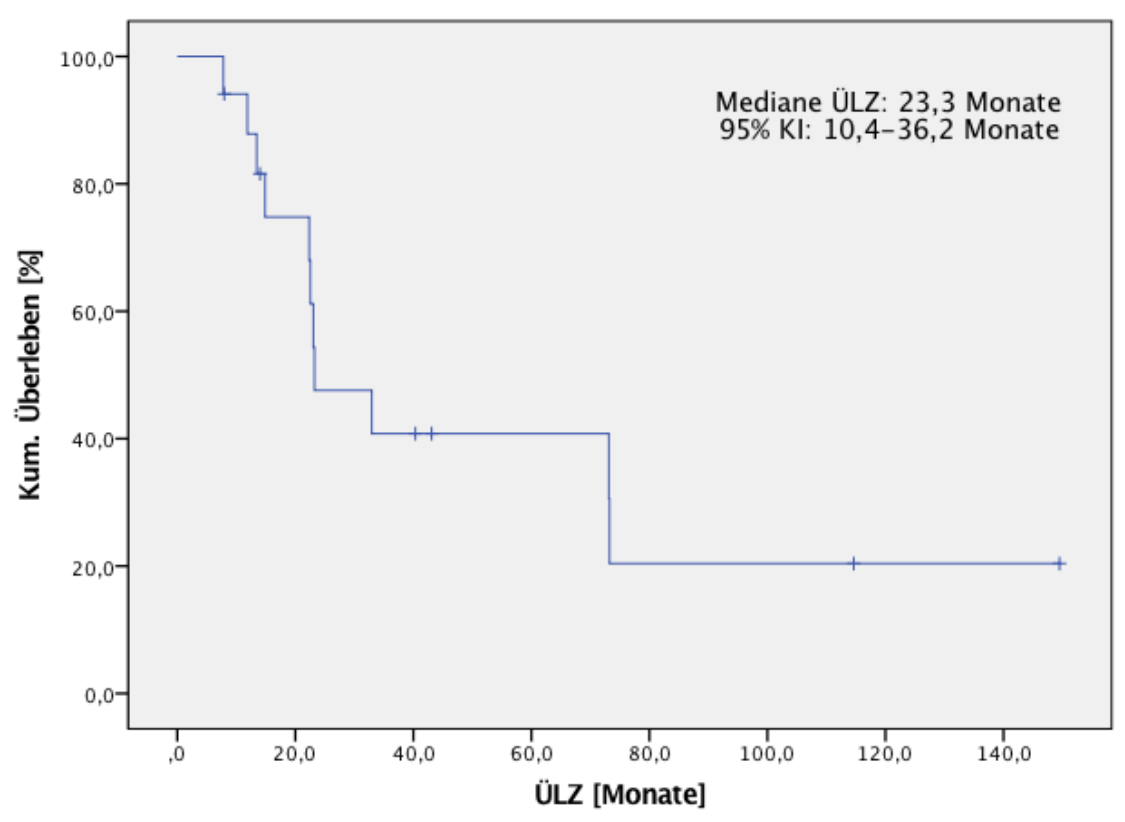

Abbildung 29: Kaplan-Meier-Überlebensfunktion für DER(1;7) $(n=17)$

3.2.30 AML-freie Überlebenszeit in der Subgruppe von Patienten mit derivativem Chromosom (1;7) (DER1;7)

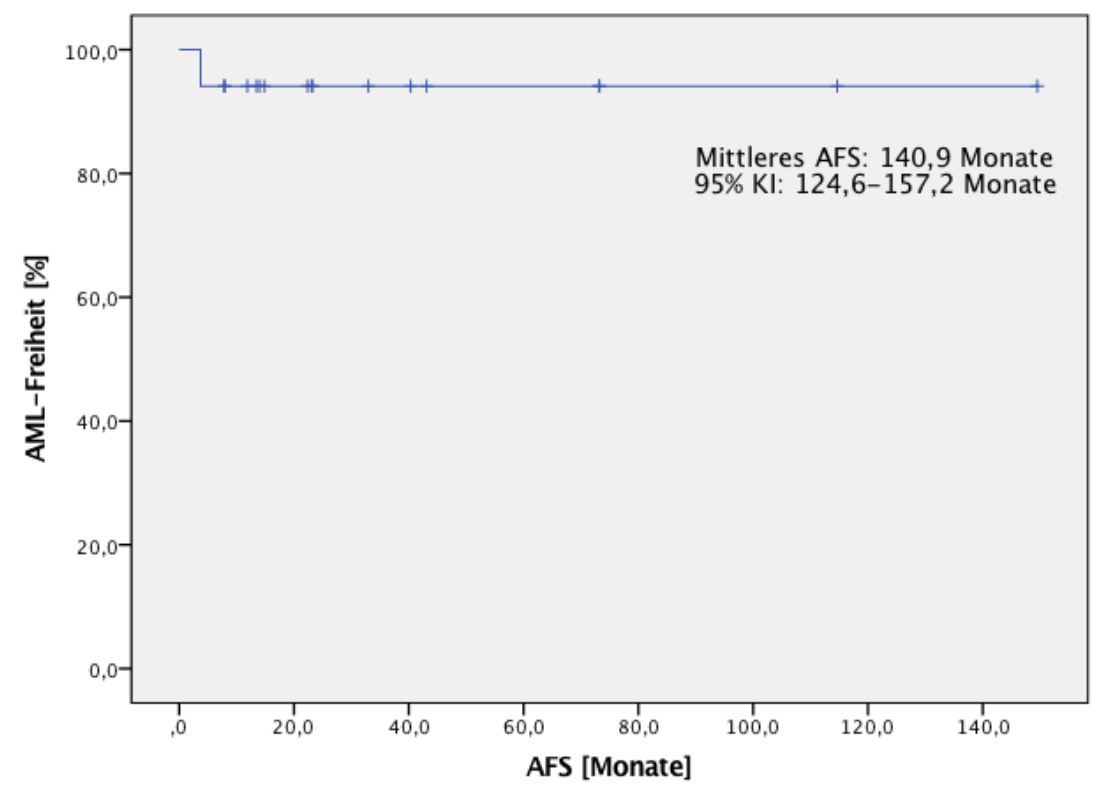

Abbildung 30: Kaplan-Meier-Funktion für AFS DER(1;7) (n=17) 


\subsubsection{1 Überlebenszeit in der Subgruppe von Patienten mit balancierten Translokationen (TRB)}

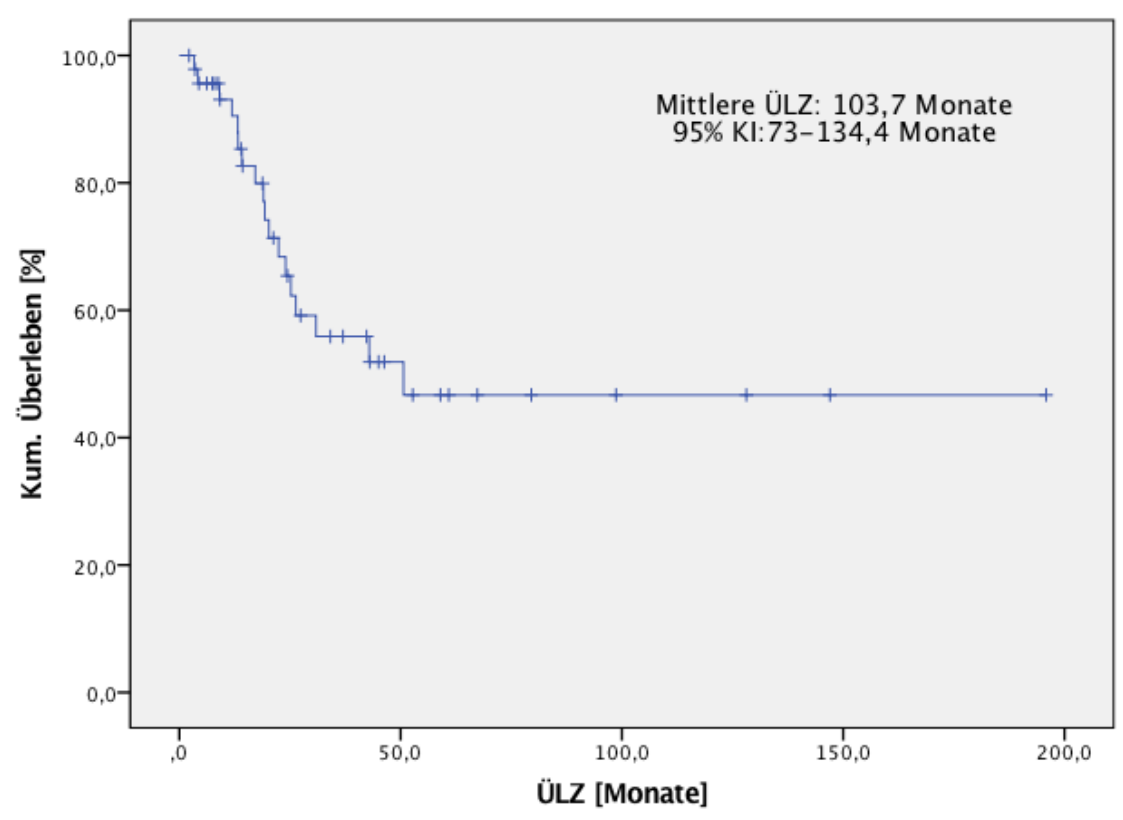

Abbildung 31: Kaplan-Meier-Überlebensfunktion für TRB (n=47)

3.2.32 AML-freie Überlebenszeit in der Subgruppe von Patienten mit balancierten Translokationen (TRB)

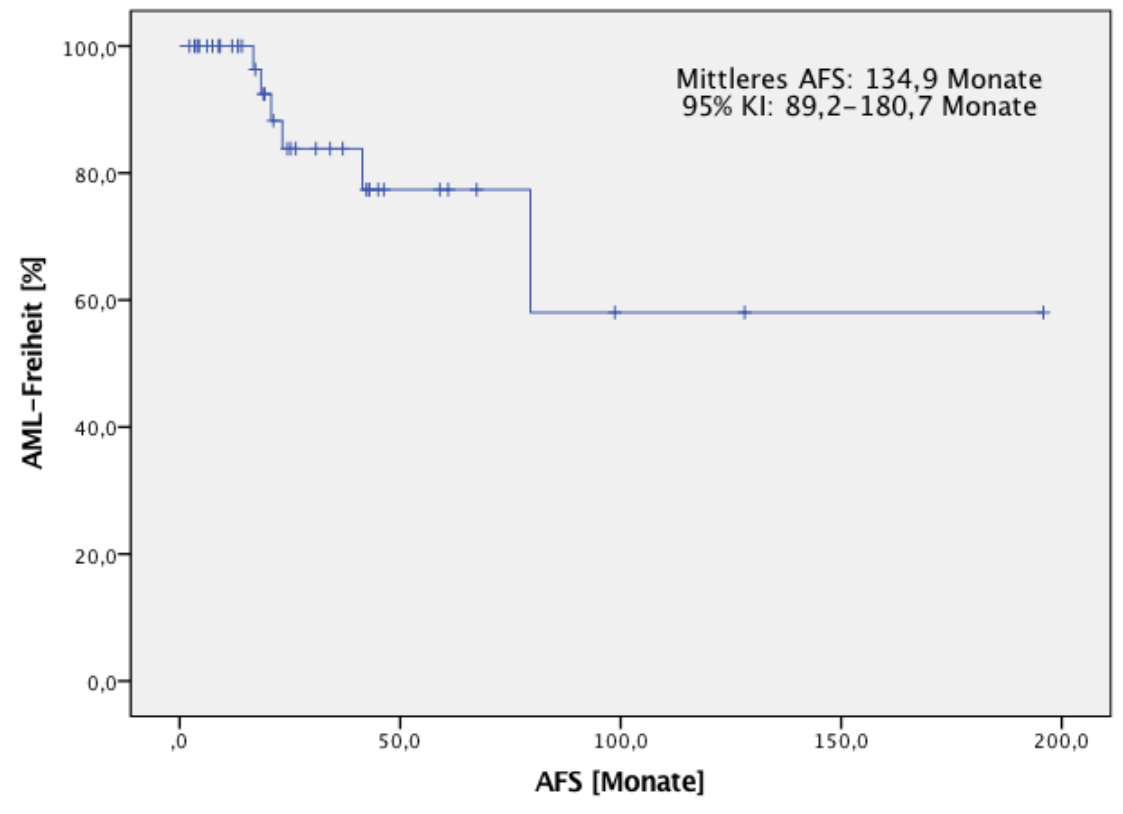

Abbildung 32: Kaplan-Meier-Funktion für AFS TRB (n=41) 
3.2.33 Überlebenszeit in der Subgruppe der Patienten mit Markerchromosom (MAR)

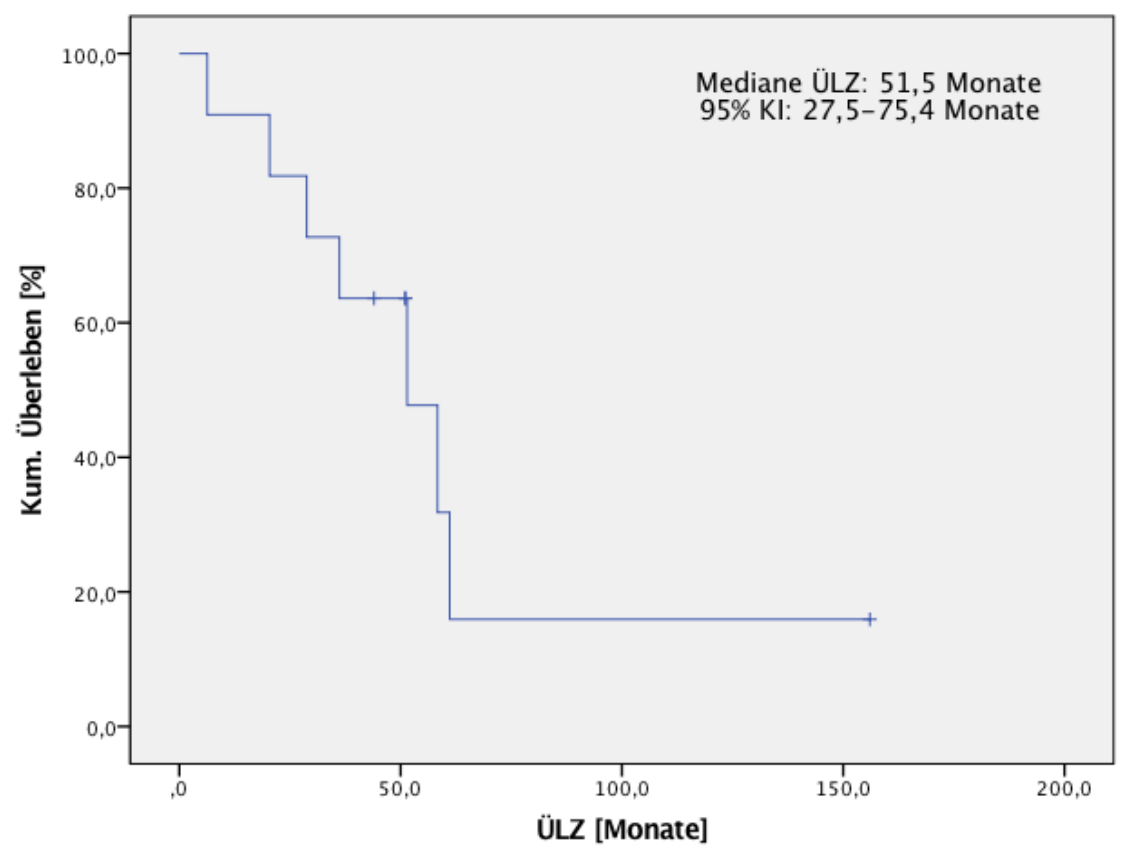

Abbildung 33: Kaplan-Meier-Überlebensfunktion für MAR (n=11)

3.2.34 AML-freie Überlebenszeit in der Subgruppe der Patienten mit Markerchromosom (MAR)

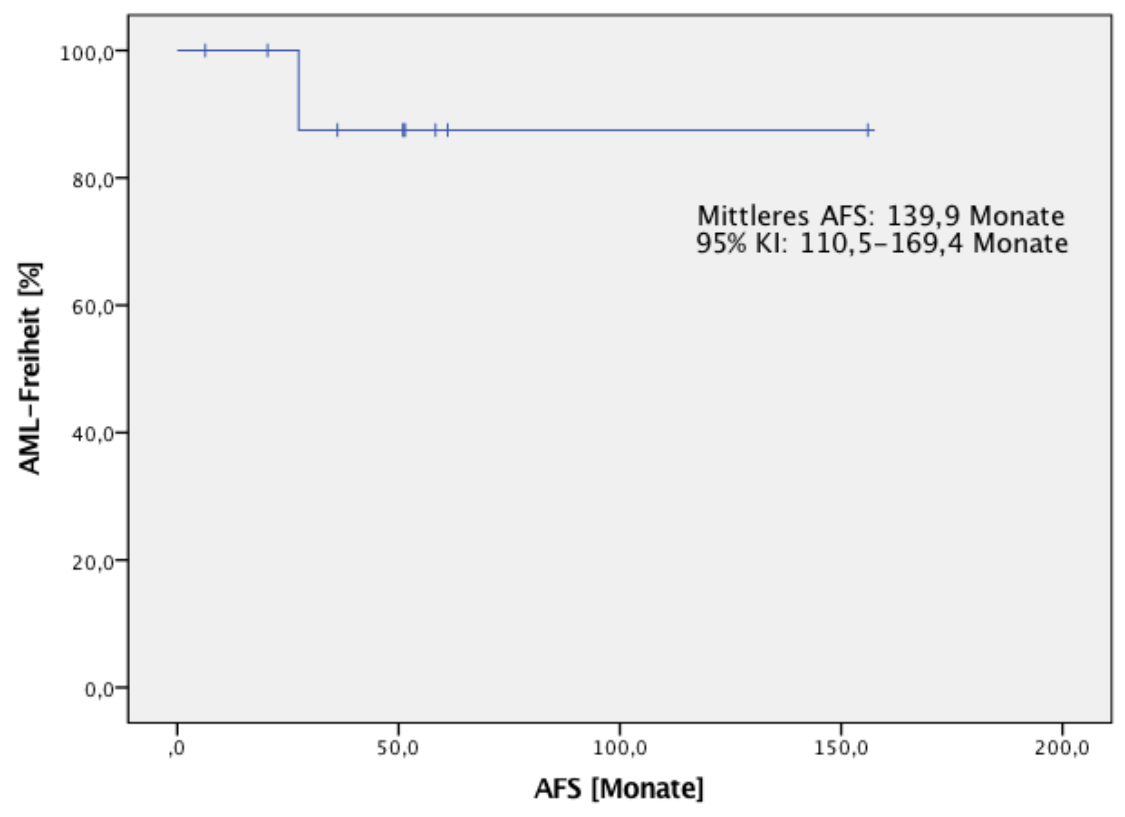

Abbildung 34: Kaplan Meier-Funktion für AFS MAR (n=10) 
3.2.35 Überlebenszeit in der Subgruppe von Patienten mit allen anderen Einzelchromosomenanomalien (OTH)

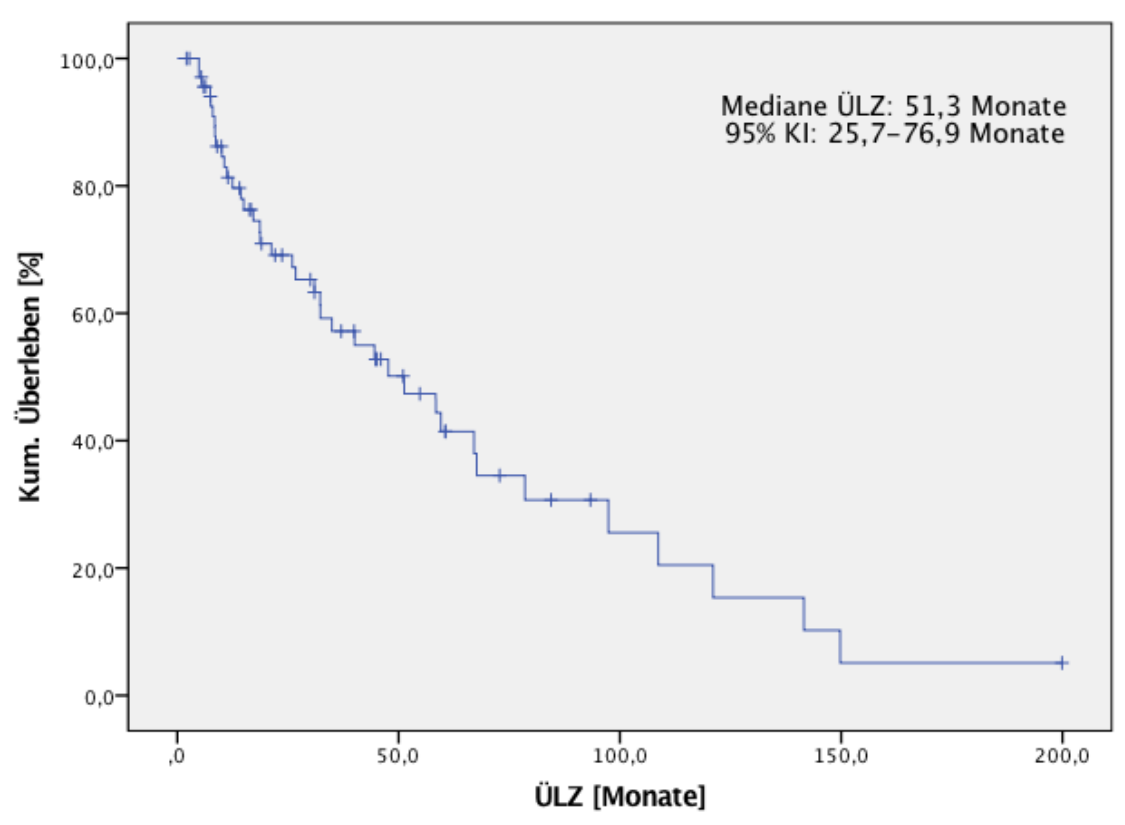

Abbildung 35: Kaplan-Meier-Überlebensfunktion für OTH (n=70)

3.2.36 AML-freie Überlebenszeit in der Subgruppe von Patienten mit allen anderen Einzelchromosomenanomalien (OTH)

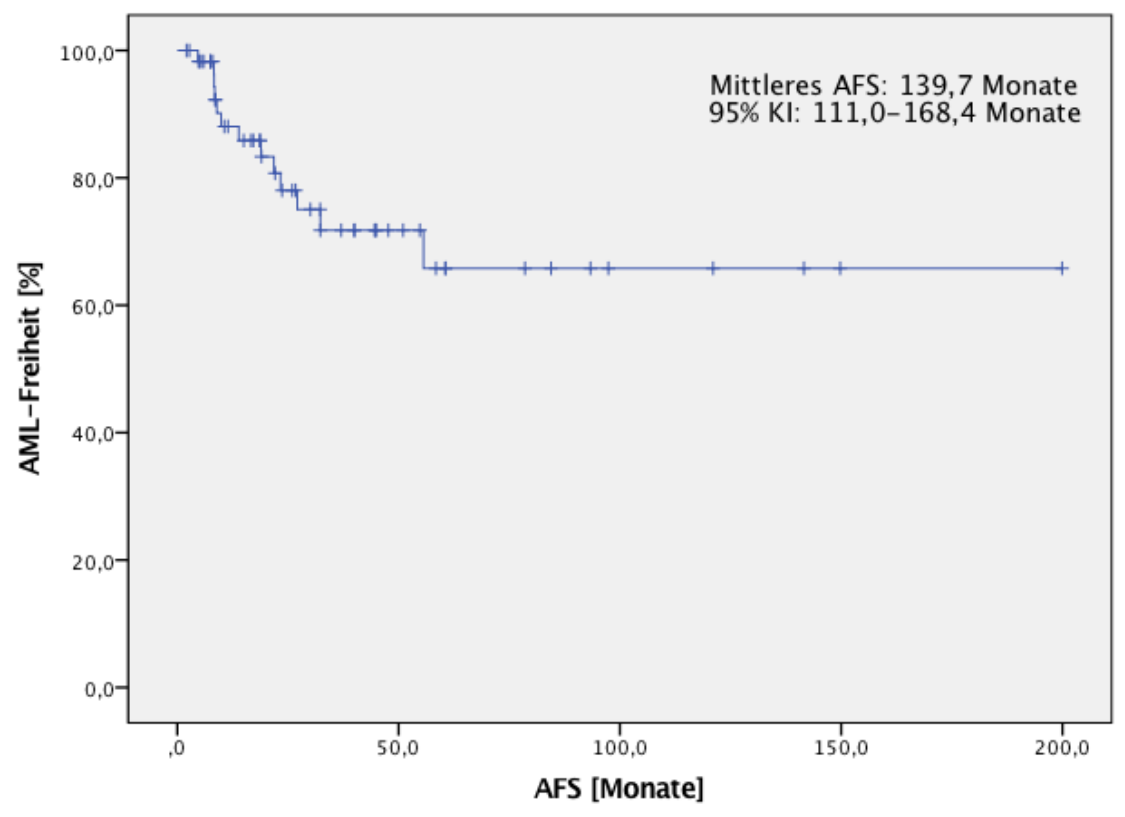

Abbildung 36: Kaplan-Meier-Funktion für AFS OTH (n=60) 


\subsection{Multivariate Überlebensanalyse der Subgruppen}

In der durchgeführten multivariaten Analyse wurde sowohl für die Überlebenszeit als auch für die AML-freie Überlebenszeit der Einfluss verschiedener Variablen untersucht.

Im Einzelnen waren dies:

- Geschlecht

- Alter

- Hämoglobin

- Leukozyten

- Thrombozyten

- Blastenanteil im Knochenmark

\subsubsection{Multivariate Analyse der Überlebenszeit}

In der multivariaten Analyse der Überlebenszeit zeigte sich, dass die bekannten Prognosefaktoren für Patienten mit myelodysplastischen Syndromen auch in dieser Analyse ihre Gültigkeit bewahrten. Männer hatten mit einer HR von 1,5 ein erhöhtes Sterberisiko gegenüber Frauen. Dies zeigte sich in Übereinstimmung mit vorangegangenen Untersuchungen (Greenberg et al. 2012). Auch das Alter ( $p=0,0001)$, der Hämoglobinwert $(p<0,0001)$, die Leukozytenzahl $(p<0,0001)$, die Thrombozytenzahl $(p=0,0104)$, sowie der prozentuale Anteil an Knochenmarksblasten $(\mathrm{p}<0,0001)$ spielten für die Überlebenszeit eine hochsignifikante Rolle.

Eine signifikant bessere Prognose hinsichtlich des Überlebens fiel für die Patienten mit Deletion $(13 q)$ mit einer HR von $0,3(p=0,015)$ auf. Ähnlich günstig fiel sie für Patienten mit Monosomie X aus. Hier wurde mit einer HR von 0,2 und einem $p=0,052$ das Signifikanzniveau nur knapp verfehlt. Mit einer HR von $0,6(p=0,046)$ zeigte sich auch ein geringeres Sterberisiko für die Gruppe der Patienten mit balancierten Translokationen.

Im Gegensatz dazu erwies sich das Sterberisiko in der Subgruppe der Patienten mit Veränderungen am Chromosom 20 mit einer HR von 2,1 $(p=0,117)$ als doppelt so hoch wie in der Kontrollgruppe. Ähnliche Tendenzen, wenn auch nicht gleichstark ausgeprägt, ließen sich für das Sterberisiko der Patienten mit totaler oder partieller Monosomie der Chromosomen 9 (HR 1,9; $\mathrm{p}=0,071), 18$ (HR 1,7; $=0,275)$ und 14 (HR1,7; $\mathrm{p}=0,476)$ nachweisen. Das Signifikanzniveau wurde hier aber jeweils nicht erreicht. 
Tabelle 8: Überlebenszeit, multivariate Analyse $(n=857)$

\begin{tabular}{|c|c|c|c|c|c|c|c|}
\hline & & Gesan & atüberle & benszeit & & & \\
\hline & & Anzahl & Median & $\begin{array}{c}\text { Hazard Ra- } \\
\text { tio }\end{array}$ & Konfid & tervall & P-Werte \\
\hline & & & [Monate] & & unteres & oberes & \\
\hline Geschlecht & Männer & 500 & & 1,5 & 1,2 & 1,8 & 0,0005 \\
\hline & Frauen & 357 & & Referen & uppe & & \\
\hline Alter & & & & 1,0 & 1,0 & 1,0 & 0,001 \\
\hline Hämoglobin & $<8 \mathrm{~g} / \mathrm{dl}$ & & & & & & \\
\hline & $8-10 \mathrm{~g} / \mathrm{dl}$ & & & 0,8 & 0,8 & 0,9 & $<0,0001$ \\
\hline & $>10 \mathrm{~g} / \mathrm{dl}$ & & & & & & \\
\hline Leukozyten & $<0,8 * 10^{3} / \mu \mathrm{l}$ & & & 1,0 & 1,0 & 1,0 & $<0,0001$ \\
\hline & $>0,8^{*} 10^{3} / \mu \mathrm{l}$ & & & & & & \\
\hline Thrombozyten & $<50 * 10^{3} / \mu \mathrm{l}$ & & & & & & \\
\hline & $50-100^{*} 10^{3} / \mu \mathrm{l}$ & & & 1,0 & 1,0 & 1,0 & 0,01 \\
\hline & $>100 * 10^{3} / \mu \mathrm{l}$ & & & & & & \\
\hline Blasten im KM & $<2 \%$ & & & & & & \\
\hline & $2-5 \%$ & & & 1,1 & 1,0 & 1,1 & $<0,0001$ \\
\hline & $5-10 \%$ & & & & & & \\
\hline & $>10 \%$ & & & & & & \\
\hline Zytogenetische & uppen & & & & & & \\
\hline & NOR & 604 & 47,4 & $\begin{array}{l}\text { Referen: } \\
\text { HR defini }\end{array}$ & $\begin{array}{l}\text { uppe, } \\
\text { als } 1,0\end{array}$ & & \\
\hline & TR 1 Q & 8 & 23,0 & 1,1 & 0,4 & 3,1 & 0,909 \\
\hline & MO 3 & 13 & 40,7 & 0,9 & 0,4 & 2,0 & 0,804 \\
\hline & MO 5 & 5 & 33,7 & 1,1 & 0,3 & 4,6 & 0,852 \\
\hline & ANY 6 & 6 & NR & 0,3 & 0,1 & 2,4 & 0,268 \\
\hline & MO 9 & 13 & 23,4 & 1,9 & 1,0 & 3,6 & 0,071 \\
\hline & ANY 11 & 8 & 47,5 & 0,6 & 0,2 & 1,6 & 0,311 \\
\hline & MO 12 & 8 & 39,1 & 1,2 & 0,5 & 3,1 & 0,709 \\
\hline & MO $13 \mathrm{Q}$ & 18 & NR & 0,3 & 0,1 & 0,8 & 0,015 \\
\hline & MO 14 & 5 & NR & 1,7 & 0,4 & 7,3 & 0,476 \\
\hline & MO 16 & 7 & NR & 0,0 & & & 0,961 \\
\hline & MO 18 & 5 & 30,7 & 1,7 & 0,7 & 4,5 & 0,275 \\
\hline & ANY 20 & 8 & 10,8 & 2,1 & 0,8 & 5,2 & 0,117 \\
\hline & TR 21 & 11 & 100,7 & 0,9 & 0,4 & 2,3 & 0,838 \\
\hline & $\mathrm{MOX}$ & 6 & 59,1 & 0,2 & 0,1 & 1,0 & 0,052 \\
\hline & DER $(1 ; 7)$ & 13 & 23,3 & 0,8 & 0,4 & 1,9 & 0,647 \\
\hline & TRB & 45 & 50,7 & 0,6 & 0,3 & 1,0 & 0,046 \\
\hline & MAR & 10 & 51,5 & 0,8 & 0,4 & 1,7 & 0,497 \\
\hline & OTH & 64 & 51,3 & 0,7 & 0,5 & 1,1 & 0,172 \\
\hline
\end{tabular}

Legende: NR - nicht erreicht 


\subsubsection{Multivariate Analyse der AML-freien Überlebenszeit}

Bei der multivariaten Analyse der AML-freien Überlebenszeit zeigten sich ebenfalls in Übereinstimmung mit vorausgegangenen Studien, dass vor allem der Anteil an Blasten im Knochenmark $(p<0,0001)$ sowie der Hämoglobinwert $(p=0,0003)$ eine signifikante prognostische Bedeutung haben.

Das Risiko einer AML-Entwicklung war in Übereinstimmung mit den Überlebensdaten dieser Subgruppe bei den Patienten mit balancierten Translokationen mit einer HR von 0,7 $(p=0,381)$ vermindert. Bemerkenswert besser fielen auch die Subgruppenanalysen für Patienten mit Markerchromosom (HR 0,5; $\mathrm{p}=0,523)$ und derivativem Chromosom $(1 ; 7)$ (HR 0,4 bei einem $\mathrm{p}=0,463)$ aus.

Die Patienten mit Veränderungen des Chromosom 20 wiesen in der multivariaten Analyse mit einer HR von 4,2 ein mehr als 4-fach erhöhtes Risiko einer AML-Entwicklung auf $(\mathrm{p}=0,057)$. Dies galt auch für die Patienten mit totaler oder partieller Deletion von Chromosom 9 mit einer HR von 3,8 ( $\mathrm{p}=0,013)$. Etwas weniger stark fiel die Risikoerhöhung bei Patienten mit totaler Monosomie 5 oder Deletion (5p) aus. Hier lag die HR bei 3,3 ( $p=0,105)$. In der Subgruppe der Patienten mit totaler oder partieller Monosomie 18 lag die HR bei 2,9 $(\mathrm{p}=0,174)$. 
Tabelle 9: AML-freie Überlebenszeit, multivariate Analyse ( $n=744)$

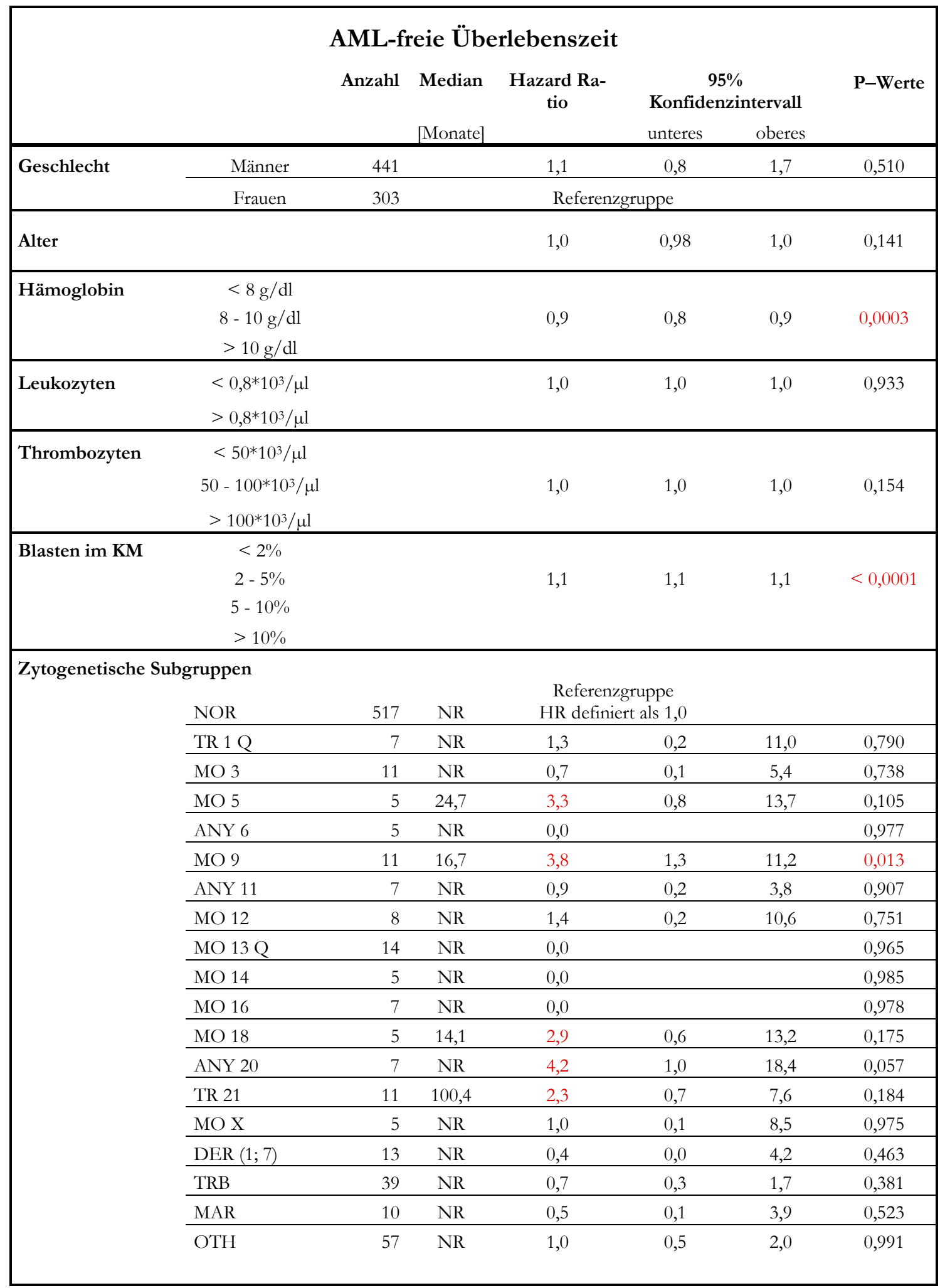

Legende: NR - nicht erreicht 


\section{Diskussion}

Die Intention der vorliegenden Arbeit bestand in der Risikostratifizierung seltener Einzelchromosomenanomalien bei myelodysplastischen Syndromen. Dies erfolgte in Form einer retrospektiven Datenanalyse an unbehandelten primären MDS, in der die Häufigkeit des Auftretens seltener Anomalien untersucht und anschließend die einzelnen Subgruppen hinsichtlich ihrer prognostischen Bedeutung für die Überlebenszeit und das AML-freie Überleben statistisch evaluiert wurden. Sinnvoll war diese Untersuchung, da in der aktuellen Fassung des IPSS-R alle seltenen Einzelchromosomenanomalien, deren günstige oder ungünstige Prognose nicht bereits verifiziert ist, in der Gruppe des intermediate risk zusammengefasst sind. Und auch wenn mehr als $90 \%$ aller zytogenetischen Veränderungen bei myelodysplastischen Syndromen bereits den verschiedenen Risikogruppen des IPSS-R zugeordnet werden können, bleibt für nahezu jeden zehnten Patienten die prognostische Bedeutung seiner zytogenetischen Aberration unklar. In der Literatur finden sich nur wenige Arbeiten zu Prognose und Entstehung einer AML bei seltenen Einzelaberrationen. Meist handelt es sich dabei um Fallserien zu isolierten Aberrationen, die gleichzeitig die jeweilige Veränderung auch in Kombination mit einer weiteren Anomalie oder im Rahmen von komplexen Anomalien untersuchen. So untersuchen beispielsweise Cortes et al. (1994) die Trisomie 21 im Hinblick auf ihre prognostische Bedeutung, Collado et al. (1999) Veränderungen am Chromosom 11; und gleich mehrere Arbeiten haben die prognostische Bedeutung der unbalancierten Translokation t(1;7) zum Gegenstand (Horiike et al. 1990; Sanada et al. 2007; Slovak et al. 2009).

Aufgrund der meist sehr geringen Fallzahlen in den publizieren Studien war eine prognostische Einordnung distinkter Anomalien bis in das 21. Jahrhundert nahezu unmöglich. Nur wenige Studien untersuchten vor dem Jahr 2000 mehr als 100 Patienten und die statistisch aussagekräftige Evaluation der Prognose war somit nur für die häufigsten Veränderungen möglich, was zu deren Einteilung in die prognostischen Subgruppen des IPSS mündete (Greenberg et al. 1997). Aussagen über die in dieser Arbeit untersuchten seltenen Anomalien finden sich in der Literatur lediglich in wenigen Arbeiten. So beschreiben White et al. (1994) an einem Kollektiv von 198 Patienten erstmals die Prognose für die Trisomie 21 und Verlust des kurzen Armes von Chromosom 12 als intermediär. Im Jahr 2000 beschrieben Solé et al. die prognostische Bedeutung von Veränderungen am langen Arm von Chromosom 1, sowie die Deletion (12p) (Solé et al. 2000). Angaben zur Prognose der Trisomie (1q), Trisomie 21, Deletion (12p) und der Monosomie 18 finden sich auch in der medizinischen Dissertation von Schanz (2003), wobei hier die Analyse nicht in der Subgruppe der isolierten Anomalien 
durchgeführt werden konnte, sondern alle Patienten mit der Anomalie umfasst, auch die Fälle mit doppelten oder komplexen Anomalien.

Größere Studien mit um die 1000 Patienten wurden in den Jahren 2005-2008 von Solé et al. (2005), Haase et al. (2007) und Pozdnyakova et al. (2008) veröffentlicht und konnten für mehrere Veränderungen die prognostische Einschätzung verbessern.

Die bislang größte Untersuchung und damit auch beste Validität für die Einschätzung der prognostischen Bedeutung seltener Einzelchromosomenanomalien bei MDS wurde von Schanz et al. (2012) durchgeführt. Doch auch in dem Studienkollektiv mit 2902 Patienten, von denen 1258 einen aberranten Karyotyp aufwiesen, traten die in der vorliegenden Arbeit untersuchten Anomalien mit weniger als zehn Fällen auf (Schanz et al. 2012).

Die vorliegende Arbeit ist die größte systematische vergleichende Analyse ausschließlich seltener Einzelchromosomenanomalien im Hinblick auf ihre prognostische Bedeutung bei primären myelodysplastischen Syndromen. Die Ergebnisse sollen helfen, für einige der seltenen Anomalien die Einschätzung der Prognose und des Risikos für die Entwicklung einer AML zu verbessern. Die Zielsetzung der vorliegenden Arbeit basiert auf der Arbeit von Schanz et al. (2012) und soll die dort aufgrund der zu geringen Fallzahl nicht mit ausreichender Genauigkeit einzuordnenden Anomalien mit Hilfe weiterer Fälle einer größeren Datenbank weiter untersuchen. Außerdem sollten zusätzliche neue Anomalien identifiziert werden. Zusätzliche Fälle der Subgruppen aus der Arbeit von Schanz et al. (2012) zu finden, gelang in der aktuellen Arbeit für die unbalancierte Translokation $\mathrm{t}(1 ; 7)$ ( $\mathrm{n}=17$ versus $\mathrm{n}=9)$, das zusätzliche Markerchromosom ( $n=11$ vs. $n=5$ ), sowie die Monosomie $X(n=7$ vs. $n=5$ ), die Trisomie (1q) ( $n=10$ vs. $n=5)$, und die Trisomie 21 ( $n=11$ vs. $n=9)$. Für die in der Arbeit von Schanz et al. (2012) zusammengefassten Anomalien Monosomie 13 und Deletion (13q) mit $\mathrm{n}=8$ Fällen konnte in der vorliegenden Arbeit mit n=20 Fällen sogar isoliert die Deletion (13q) untersucht werden. Nach Zusammenfassung aller Veränderungen am Chromosom 11 konnte in dieser Gruppe die Fallzahl der bisher ausschließlich untersuchten Trisomien (+11) am Chromosom 11 von fünf auf immerhin acht Fälle gesteigert werden. Andere Anomalien zeigten aufgrund der Größe der Datenbank erstmals ausreichend viele Fälle für eine Analyse. Dies betraf die Gruppen mit partiellem oder vollständigem Verlust der Chromosomen 3, 5, $9,12,14,16$ und 18.

Hinsichtlich des Studienkollektivs zeigt sich bei der Verteilung der Fälle einerseits die aus anderen Studien bekannte Verteilung der MDS-Subtypen entsprechend FAB- und WHOKlassifikation, außerdem ein mit 67 Jahren typischer Altersmedian bei Erstdiagnose, sowie 
die bekannte Prädominanz männlicher Patienten mit einer HR von 1,5 und deren verkürzte Überlebenszeit.

Da für gut ein Viertel der Patienten lediglich die zytogenetischen Befunde, nicht aber die klinischen Basisdaten und Verlaufsuntersuchungen zur Verfügung standen, konnten in den statistischen Analysen deutlich weniger Patienten untersucht werden, als bei der Analyse der Häufigkeiten. Somit sind die 95\%-Konfidenzintervalle der univariaten Analysen aufgrund kleiner Fallzahlen oft sehr groß. Trotzdem zeigen sich die im IPSS-R validierten Risikofaktoren und Grenzwerte für die Zytopenien auch in der vorliegenden multivariaten Analyse für das Gesamtüberleben hochsignifikant. Und, wie zu erwarten, behalten Hämoglobingehalt und Anteil der Knochenmarksblasten in der multivariaten Analyse der AML-freien Überlebenszeit ihre signifikante Bedeutung.

Im Folgenden werden die Ergebnisse entsprechend den gebildeten zytogenetischen Gruppen anhand der verfügbaren Literatur diskutiert.

\subsection{Untersuchte zytogenetische Anomalien}

\subsubsection{Trisomie (1q)}

Bei der Entwicklung einer Trisomie (1q), also der partiellen oder totalen Verdopplung des langen Armes von Chromosom 1, dominieren bei myelodysplastischen Syndromen die Duplikation von Teilen des langen Armes oder ein Zugewinn an Material am Chromosom 1 durch entsprechende Deletionen verschiedener chromosomaler Partner, die in der Bildung unbalancierter Translokationen resultieren. Verschiedene Fallbeschreibungen existieren in der Literatur, die verschiedene meist unbalancierte Translokationen unter Beteiligung des langen Armes von Chromosom 1 beschreiben. In diesen Fallbeschreibungen wird deren Vorkommen bei zum Teil unterschiedlichen myeloischen Erkrankungen beschrieben. Wang et al. (2001) beschreiben beispielsweise zwei Fälle einer der(1;18). Die unbalancierte Translokation $\mathrm{t}(1 ; 18)$ war jeweils die einzige nachgewiesene zytogenetische Anomalie. Darunter war ein Fall mit MDS und ein Fall einer nicht näher differenzierten myeloproliferativen Neoplasie (MPN) mit klinisch unterschiedlichen Verläufen. Der MDS-Fall zeigte eine rasche Progression in eine akute myeloische Leukämie, der Patient mit MPN war seiner Grunderkrankung entsprechend deutlich länger klinisch stabil. Lunghi et al. (2010) beschreiben insgesamt acht Fälle einer unbalancierten Translokation $t(1 ; 16)$, bei denen es sich ausschließlich um Männer gehandelt hat. In dieser Fallserie wurde auch eine hohe Rate sekundärer akuter myeloischer Leukämien (vier von sieben Patienten) beschrieben. Eine Reihe von Fällen mit der unbalancierten Translokation $\mathrm{t}(1 ; 5)$ bei verschiedenen myeloischen 
und lymphatischen hämatologische Erkrankungen (AML, MDS, MPN, chronisch myeloische Leukämie, akute und chronische lymphatische Leukämie) beschreiben Johanssen et al. (1997). Die Kollegen beobachteten für diese Veränderung einen Zusammenhang mit vorangegangenen Behandlungen mit Alkylanzien oder Thorotrast und ebenfalls prognostisch ungünstige Verläufe (Johansson et al. 1997).

Und auch wenn partielle oder totale Trisomien am langen Arm von Chromosom 1 beim MDS hauptsächlich als im Verlauf auftretende Veränderung dokumentiert werden (Bacher et al. 2015) oder besonders häufig Teil komplexer Anomalien sind (Haase et al. 2007), konnten wir in der dieser Untersuchung zugrundeliegenden Datenbank zehn Fälle mit Trisomie (1q) als Einzelaberration hinsichtlich der Überlebenszeit und sieben Fälle im Hinblick auf die AML-freie Überlebenszeit analysieren, was der bisher größten analysierten Gruppe bei primären myelodysplastischen Syndromen mit dieser Anomalie entspricht.

Bei den hier in der Subgruppe TR1Q analysierten Fällen handelt es sich um eine Zusammenfassung der Fälle mit Trisomie 1, Trisomie (1q), dup(1q) und unbalancierten Translokationen zwischen den verschiedenen Partnerchromosomen (12,15, 18 und 21). Das vorbeschriebene niedrige Erkrankungsalter von nur 36,5 Jahren bei 11 untersuchten Patienten mit einer Trisomie (1q) (Fonatsch et al. 1991) konnte in der vorliegenden Subgruppe nicht gezeigt werden.

Die in der Literatur anhand von Fallbeschreibungen als prognostisch ungünstig gewerteten Veränderungen der dup1(21q32q), der(1;16) und der(1;5) kamen bei unseren Fällen jedoch nicht vor. Möglicherweise ist für die Prognose auch der Kombinationspartner bei der Bildung der unbalancierten Translokation entscheidend, oder aber die duplizierte Region, wie in der Arbeit von Alfaro et al. (2008) vermutet. Da bei der Bildung von unbalancierten Translokationen eine Vielzahl unterschiedlicher Bruchpunkte vorkommen, resultiert aus den unbalancierten Translokation selbst möglicherweise eine chromosomale Instabilität, die für den weiteren Verlauf entscheidend ist, und nicht der Chromosomenbruch an sich bestimmt die Prognose (Lunghi et al. 2010).

Für die Trisomie (1q) konnte in der vorliegenden Arbeit eine mittlere Überlebenszeit von 38,1 Monaten bei insgesamt zehn Fällen ermittelt werden. Damit liegt die Zeit deutlich höher als in der Analyse von Schanz et al. (2012) mit 21,3 Monaten. In beiden Untersuchungen ist sie deutlich länger als die in den Untersuchungen von Solé et al. (2000 und 2005) mit eine medianen Überlebenszeit von 4 respektive 8 Monaten. In den beiden erwähnten Studien der spanischen Arbeitsgruppe gingen dabei in die Analyse allerdings nicht nur Fälle mit totaler oder partieller Trisomie (1q) oder Duplikation (1q) ein, sondern auch andere Veränderungen am langen Arm von Chromosom $1(n=4)$. Eine schlechte Prognose bescheinigen auch Alfaro 
et al. (2008) in diesem Fall der dup1(q21q32), da die beiden beschriebenen eigenen Fälle eine rasche Krankheitsprogression mit entweder einer zusätzlichen Trisomie 8 beziehungsweise die Entwicklung einer sekundären AML zeigten (Alfaro et al. 2008). Inwieweit die geringe Fallzahl oder die Einzelfallbeschreibung die stark differenten Ergebnisse erklären können, bleibt letztlich fraglich. Allerdings sind unsere Überlebensdaten in guter Übereinstimmung mit der Untersuchung von Haase et al. (2007), in der anhand weniger Patienten (0,4\% des Gesamtkollektivs) eine mediane Überlebenszeit von 34,7 Monaten errechnet werden konnte. In dieser Analyse wurden Patienten mit Trisomie 1 und Trisomie (1q) innerhalb eines nichtkomplexen Karyotyps untersucht, d.h. zusätzlich zur partiellen oder totalen Trisomie 1 lag maximal eine weitere Anomalie vor (Haase et al. 2007). Trotz Hinzunahme der Patienten mit einer zusätzlichen Anomalie ergeben sich vergleichbare Überlebenszeiten. Eine erneute Analyse nicht nur der als Einzelanomalie auftretenden Fälle, sondern auch der mit einer zusätzlichen Anomalie auftretenden Trisomie (1q) könnte gegebenenfalls Aufschluss über die Prognose mit statistischer Relevanz erbringen. Hinsichtlich der in der Literatur beschriebenen hohen Rate an sekundären AML (Alfaro et al. 2008; Lunghi et al. 2010) ist auch in unserer Untersuchung auffällig, dass sich mit einer HR von 1,3 eine Tendenz zu kürzerem AML-freiem Überleben zeigt. Allerdings wird bei 7 Patienten in der Analyse auch hier keine statistische Relevanz erreicht, sodass aufgrund der geringen Gruppengröße keine verlässliche Aussage möglich ist. Die einzige andere Arbeit, die die AML-freie Überlebenszeit analysiert, ist die Arbeit von Schanz et al. (2012); gegenüber den dort veröffentlichten Daten ist die AML-freie Überlebenszeit nach den Erkenntnissen der vorliegenden Arbeit etwas mehr als doppelt so lang (19,2 vs. 45,9 Monate). Auch die Berechnungen der Überlebenszeit verfehlen in der vorliegenden multivariaten Analyse trotz verdoppelter Fallzahl gegenüber Schanz et al. (2012) das statistische Signifikanzniveau. Hinsichtlich des IPSS-R liegt die ÜLZ unserer Subgruppe mit 38,1 Monaten nahe der medianen ÜLZ der Patienten im IPSS-R mit intermediären Risiko (32,4 Monate), sodass aufgrund dieser neuen Daten nichts gegen den Verbleib der Trisomie (1q) in der Gruppe des intermediären Risikos nach IPSS-R spricht.

\subsubsection{Unbalancierte Translokation $\mathrm{t}(1 ; 7)$}

Unabhängig von den übrigen unbalancierten Translokationen wurde in unserer Untersuchung die unbalancierte Translokation $\mathrm{t}(1 ; 7)$ analysiert, welche durch Verlust des langen Armes am Chromosom 7 und einem Materialzugewinn am langen Arm von Chromosom 1 entsteht. Die unbalancierte Translokation t(1;7) konnte in einem Kollektiv von 1377 Fällen bei de-novo-MDS in 1,8\% der Fälle nachgewiesen werden (Mauritzson et al. 2002). Da bei 
der Translokation auch eine Deletion (7q) entsteht, liegt aufgrund der Daten zur Monosomie 7 und Deletion (7q) mit ihrer deutlich schlechteren Prognose bei myelodysplastischen Syndromen und Einordnung in die poor risk zytogenetische Gruppe nach IPSS-R (Greenberg et al., 2012) auch im Fall der der(1;7) ein negativer Einfluss auf die Überlebenszeit und das AML-freie Überleben nahe. Während in der Literatur sowohl Hinweise für eine schlechte Prognose zu finden sind (Horiike et al. 1990) mit einem medianen Überleben bei sechs Patienten von nur 4 Monaten, vermuteten Sanada et al. (2007) ein besseres medianes Überleben und auch besseres AFS verglichen mit Patienten mit Monosomie 7 und Deletion (7q), was sich in der Untersuchung von Slovak et al. (2009) wiederum nicht in gleicher Weise reproduzieren ließ. Hier ergaben sich hinsichtlich der Prognose der der(1;7) keine signifikanten Unterschiede zur schlechten Prognose der Patienten mit Monosomie 7 und Deletion (7q) (Slovak et al. 2009).

In der vorliegenden Arbeit konnten 17 Fälle hinsichtlich ihrer Überlebenszeit ausgewertet werden. Während in der Arbeit von Schanz et al. (2012) weder die mediane Überlebenszeit noch die AML-freie Überlebenszeit für die unbalancierte Translokation $\mathrm{t}(1 ; 7)$ anhand der analysierten neun Patienten erreicht wurden, liegt in der aktuellen univariaten Analyse die Überlebenszeit mit 23 Monaten nur leicht über der der anderen Veränderungen (Monosomie 7 mit 15.8 Monaten und Deletion (7q) mit 20 Monaten) (Schanz et al. 2012). Allerdings ergibt sich in unserer multivariaten Analyse bei einer HR von $0,82(p=0,647)$ für die der $(1 ; 7)$, eine Tendenz zu einer besseren Überlebenszeit, verglichen mit der Kontrollgruppe mit normalem Karyotyp (47,4 Monate).

Noch ausgeprägter fiel dieser Effekt bei der multivariaten Analyse der AML-freien Überlebenszeit aus. Hier zeigte sich in der vorliegenden Arbeit eine HR von 0,43 ( $p=0,463)$, was auf eine deutlich verlängerte AML-freie Überlebenszeit hindeutet. Bereits 2008 fanden Pozdnyakova et al. für sechs Patienten mit der(1;7) eine mediane Überlebenszeit von 45,5 Monaten. Keiner der Patienten entwickelte eine AML (Pozdnyakova et al. 2008). Diese Ergebnisse passen zu denen von Ganster et al. (2015), die bei einer Analyse von 31 Pat. mit $\operatorname{der}(1 ; 7)$ alleine und vier Pat. mit nur einer zusätzlichen Anomalie ein medianes Überleben von 53,4 Monaten zeigen konnte und in deren Arbeit die AML-freie Überlebenszeit wie in der Arbeit von Schanz et al. (2012) ebenfalls nicht erreicht wurde (Ganster et al. 2015). Hsiao et al. (2006) gehen ebenfalls nach einer Analyse von 23 Pat. mit der(1;7) mit MDS und AML davon aus, dass die ungünstige Prognose eher durch das Vorliegen weiterer zytogenetischer Veränderungen oder durch vorangegangene zytotoxische Therapien (Radiatio, Therapie mit Antimetaboliten) erklärt werden muss, als durch die der(1;7) alleine (Hsiao et al. 2006). 
Hierzu passen auch die Ergebnisse der Analyse von Sanada et al. (2007), die beim Auftreten mehrerer zytogenetischer Subklone die unbalancierte Translokation $\mathrm{t}(1 ; 7)$ sowohl in allen veränderten Metaphasen als auch jeweils bei Erstdiagnose nachweisen konnten, sodass hier von einer hohen zytogenetischen Stabilität des Klons ausgegangen werden kann (Bacher et al. 2015), die die geringe Rate an sekundären AML in verschiedenen Analysen möglicherweise erklärt. Trotzdem bleibt auch in der gegenwärtigen Arbeit bei positiven Trends in der multivariaten Analyse das Problem der fehlenden Signifikanz, sodass die bessere als intermediäre Prognose der der $(1 ; 7)$ vor allem vor dem Hintergrund der ergänzenden Literatur zwar plausibel erscheint, aber weiterhin als unsicher gelten muss.

\subsubsection{Monosomie 3}

In der statistischen Analyse der Patientengruppe mit partieller oder totaler Monosomie 3 konnten wir zeigen, dass sich bei immerhin 14 Fällen die mediane Überlebenszeit mit 40,7 Monaten nicht statistisch von der Kontrollgruppe unterscheidet (HR von 0,9; $\mathrm{p}=0,804$ ). Hinsichtlich der AML-freien Überlebenszeit scheint bei einer HR von 0,71 $(p=0,738)$ eine AML hier eher seltener aufzutreten $(\mathrm{n}=12)$. Die mediane Zeit bis zum Auftreten einer AML wurde nicht erreicht. Allerdings wird auch hier das Signifikanzniveau nicht erreicht.

Bisher wurden die partiellen und totalen Monosomien des Chromosom 3 in der Literatur nicht gesondert betrachtet, sodass bisher über die Prognose für MDS-Patienten mit isolierter Monosomie 3, Deletion (3q) und Deletion (3p) nichts bekannt ist, um diese Ergebnisse zu verifizieren. Wenige Fälle mit Deletion (3q) waren in der Untersuchung von Schanz et al. (2012) mit den Patienten mit Rearrangments (inv(3) und t(3q)) zusammengefasst. In dieser Arbeit hatten die Patienten dann auch nur eine mediane Überlebenszeit von 23,8 Monaten und ein AML-freies Überleben von 7,9 Monaten und wurden dem entsprechend in die zytogenetische Gruppe des poor risk nach IPSS-R eingeordnet.

Rearrangements am Chromosome 3 unter Einbeziehung der Banden q21 und q26 wurden bereits in den 80er und 90er Jahren des letzten Jahrhunderts als ein spezifischer Subtyp myeloider Neoplasien mit kurzer Überlebenszeit, schlechtem Therapieansprechen und schlechter Prognose bei hoher Inzidenz zusätzlicher Anomalien am Chromosom 7 beschrieben (Jotterand Bellomo et al. 1992). Bei der Pathogenese dieser speziellen Entität scheint die durch verschiedene Rearrangements ausgelöste Überexpression bestimmter auf Bande 3q26 lokalisierter Gene (EVI1/MECOM) eine Rolle zu spielen (De Braekeleer et al. 2015; Baldazzi et al. 2016).

Neben der Arbeit von Solé et al. (2005), in der für die Patienten mit Rearrangements von 3q21q26 ( $n=6)$ eine intermediäre Prognose mit einer medianen Überlebenszeit von >30 Monaten, bei gleichzeitigem 75\%-AML-Progressionsrisiko binnen eines Jahres errechnet wurde, stellen auch Haase et al. (2007) isolierte Berechnungen für die mediane Überlebenszeit von Patienten mit Rearrangements von Chromosom (3q) an: Die Patienten mit Rearrangements am langen Arm von Chromosom 3 ( $\mathrm{n}=16)$ hatten hier eine mediane Überlebenszeit von nur 
19,9 Monaten, Patienten mit allen anderen Veränderungen am Chromosom 3 zusammengefasst nur ein medianes Überleben von 17,1 Monaten. Welche Veränderungen genau hier zusammengefasst wurden, ist der Publikation allerdings nicht zu entnehmen. Damit sind die Ergebnisse der Arbeit von Haase et al. (2007) mit den Berechnungen von Schanz et al. (2012) und auch mit den Ergebnissen von Pozdnyakova et al. (2008) hinsichtlich des medianen Überlebens der Patienten mit Rearrangements vergleichbar, auch wenn in der letztgenannten Arbeit nur drei Patienten die entsprechenden Veränderungen aufwiesen.

Die neuen Daten dieser Arbeit bestätigen die bisher angenommene schlechte Prognose bei fehlender direkter Vergleichbarkeit der Studien nicht. Da erstmals an einem großen Kollektiv die Untersuchung einer Subgruppe mit ausschließlichen Deletionen am Chromosom 3 möglich war, stellen die Daten mit einer zu vermutenden besseren Prognose (nahe den Patienten mit normalem Karyotyp) gegenüber den Rearrangements am langen Arm des Chromosom 3 ein Novum dar.

\subsubsection{Monosomie 5}

Auffällig ist in dieser Gruppe vor allem die geringe Anzahl der Fälle ( $\mathrm{n}=5)$. Denn obwohl die Monosomie 5 als Teil eines komplex-aberranten Karyotyp bei myelodysplastischen Syndromen häufig ist, finden sich doch nur wenige Fälle in der dieser Arbeit zugrunde liegenden Datenbank primärer MDS-Patienten bei der Suche nach Einzelanomalien. Haase et al. (2007) beschreiben die Monosomie 5 in ihrem Kollektiv von 2072 Pat. mit einer Frequenz von 6\%, dabei aber nur in 0,4\% der Fälle in einem nicht-komplexen Karyotyp, d. h. als Einzelanomalie oder mit maximal einer weiteren Anomalie. Diese Analyse deckt sich mit den Ergebnissen von Katarjian et al. (2009). Von insgesamt 2743 Patienten der Studie hatten n=503 (18\%) Veränderungen am Chromosom 5. Davon entfielen $n=287$ (10\%) auf die del(5q), die bei bereits bekannter Prognose nicht Gegenstand dieser Arbeit ist, und $n=216$ (8\%) auf die Monosomie 5. Von diesen hatte aber nur ein Patient die Monosomie 5 als Einzelanomalie, ein weiterer mit einer und fünf weitere Patienten mit zwei weiteren Anomalien. Bei 67 Patienten ließ sich die Monosomie 5 im Rahmen eines komplex-aberranten Karyotyps nachweisen (drei oder mehr zusätzlichen Anomalien), und bei 142 Patienten waren zusätzliche Veränderungen am Chromosom 7 nachweisbar (Kantarjian et al. 2009). Gleichzeitig waren in dieser Studie die Fälle mit Monosomie 5 häufiger mit fortgeschritteneren MDS und höherem IPSS (insgesamt 93\% intermediate II und High risk) assoziiert, sowie mit anderen genetischen Veränderungen und Veränderungen am Chromosom 7. Bei Niedrig-Risiko-MDS trat die Monosomie 5 dagegen nur äußerst selten auf (Kantarjian et al. 2009).

Diesen Zusammenhang beschreibt bereits Heim (1992) in einer Metaanalyse von 1300 Fällen mit primärem und sekundärem MDS. Dort war ebenfalls die del $(5 q)$ mit primärem MDS assoziiert, während die Monosomie 5 eine der häufigsten Anomalien bei therapieassoziierten 
MDS war. In Übereinstimmung damit spricht die Verteilung der Fälle mit Monosomie 5 in der Studie von Kantarjian et al. (2009) an primären MDS für eine hohe Instabilität der Monosomie 5 und rasche klonale Evolution dieser Anomalie hin zu komplexen Veränderungen mit entsprechend schlechter Prognose.

Unsere wenigen Fälle wären dann möglicherweise genau in dem Augenblick der Monosomie 5 ohne weitere Anomalien diagnostiziert worden. Da aber in der vorliegenden Arbeit die klonale Evolution mittels wiederholter zytogenetischer Untersuchungen der Patienten nicht untersucht wurde, muss diese Vermutung eine Hypothese bleiben. Möglich wäre auch, dass aufgrund der hohen Instabilität dieser Anomalie Monosomien von Chromosom 5 gar keine echten Monosomien darstellen, sondern durch Zersplitterung des Chromosoms und Anlagerung des genetischen Materials an andere Chromosomen eine Vielzahl an unbalancierten Translokationen im Rahmen einer „Chromothripsis“ resultieren (Zemanova et al. 2014).

Diesen Zusammenhang postulieren Zemanova et al. (2014), da sich in ihrer an 157 Patienten mit neu diagnostiziertem MDS und komplex-aberrantem Karyotyp in der Genanalyse keine einzige echte Monosomie 5 nachweisen ließ, sondern eine Vielzahl kryptischer Rearrangements unter Beteiligung von Chromosom 5 gefunden wurden. Dies wäre eine Erklärung für die hohe HR von 3,3 ( $\mathrm{p}=0,105)$ bei der AML-freien Überlebenszeit in unserer Untersuchung. Diese war mit 24,7 Monaten verglichen mit der Untersuchung von Schanz et al. (2012) eher in der Gruppe der Patienten mit drei Anomalien anzusiedeln und nicht in der Gruppe der Patienten mit Einzelanomalie (21 vs. 203 Monate). Gleichwohl zeigt sich die Überlebenszeit in der multivariaten Analyse nicht im selben Maße verändert. Hier ergibt die HR von 1,1 nur eine sehr geringe Tendenz zu verkürztem Überleben. Das mediane Überleben ist mit 33,7 Monaten im Vergleich zur Kontrollgruppe zwar deutlich kürzer, statistische Signifikanz besteht hier aber nicht. Dies gelang auch in der Untersuchung von Haase et al. (2007) nicht, wo für die Monosomie 5 im Rahmen eines nicht-komplexen Karyotyps ein medianes Überleben von 14,9 Monaten erreicht wurde, allerdings ebenfalls nicht mit hinreichender statistischer Validität. Allerdings tritt auch in dieser Analyse die Monosomie 5 in 92,8\% aller Fälle im Rahmen komplexer Anomalien auf (Haase 2008).

So bleibt die Prognose der Monosomie 5 weiterhin unsicher, die breitere Anwendung von Gensequenzierungsverfahren kann aber möglicherweise dabei helfen, die Pathogenese der Monosomie 5 weiter zu klären und dann auch zu einer verbesserten Einschätzung der Prognose zu kommen. 


\subsubsection{Veränderungen am Chromosom 6}

Über die Prognose von Patienten mit Veränderungen am Chromosom 6 ist nur wenig bekannt. Es existieren einzelne Fallbeschreibungen über Patienten mit Deletion (6q), die zum einen bei Vorliegen der Veränderung und einer Refraktären Anämie von stabilen Krankheitsverläufen über 10 und 12 Monate berichten (Gozzetti et al. 2009; Ferdowsi et al. 2013), aber eben auch ein Patient mit einer RAEB-t und Deletion (6q), der rasch in eine AML überging (De Souza Fernandez et al. 2000). In der Analyse von Haase et al. (2007) an 2072 Patienten schließlich, ließ sich aufgrund der Seltenheit, mit der Veränderungen am Chromosom 6 als Einzelanomalie auftreten, ebenfalls keine Aussage über die Prognose treffen. In der vorliegenden Arbeit gestaltete sich das Problem trotz eines größeren Patientenkollektivs noch immer ähnlich. Das verdeutlicht vor allem die Seltenheit dieser Anomalie. Auch in unserer Untersuchung traten nur sechs Fälle von Einzelanomalien am Chromosom 6 auf. Nach Ausschluss der Translokationen in dieser Gruppe verblieben fünf Patienten mit einer del(6q), sowie ein Patient mit del(6p). Das Überleben in dieser Gruppe ist jedoch deutlich besser als in der Kontrollgruppe mit einer HR von 0,33 ( $p=0,268)$. Fälle von AML traten hier dem entsprechend nicht auf. Das führt zu der These, dass es sich bei der del(6q) um eine prognostisch günstige Veränderung handeln könnte. Diese Ergebnisse sind aufgrund der ausgesprochen wenigen Fälle natürlich sehr vorsichtig zu interpretieren.

\subsubsection{Monosomie 9}

Bezüglich der Monosomie 9 ergeben sich in unserer Analyse interessante Aspekte. Bei insgesamt 14 Fällen mit überwiegend $\operatorname{del}(9 \mathrm{q})$ ergibt sich für die mediane Überlebenszeit mit 23,4 Monaten eine Tendenz zu schlechterem Überleben dieser Patienten mit einer HR von 1,8. Das Signifikanzniveau wird hier mit $\mathrm{p}=0,071$ knapp verfehlt. Die AML-freie Überlebenszeit ist mit einer HR von 3,8 sogar nahezu 4-fach kürzer als in der Kontrollgruppe (AFS von 29 Monaten). Diese Analyse war signifikant ( $p=0,013)$. Allerdings sind diese Daten nicht konkordant mit den Daten von Haase et al. (2007), in der die del $(9 \mathrm{q})$, nachgewiesen bei $0,4 \%$ der Fälle innerhalb eines nicht-komplexen Karyotyps in die prognostisch günstige zytogenetische Gruppe fällt, da die mediane Überlebenszeit bei den untersuchten wenigen Patienten nicht erreicht wurde.

In der Untersuchung von Pozdnyakova et al. (2008) konnten unter 1029 Patienten nur zwei Fälle einer $\operatorname{del}(9 \mathrm{q})$ als Einzelanomalie nachgewiesen werden. Diese hatten eine Überlebenszeit von 12 und 26 Monaten und wurden als prognostisch intermediär eingestuft. In der bisher größten Untersuchung von Schanz et al. (2012) zu Einzelchromosomenaberrationen 
findet sich keine Analyse der $\operatorname{del}(9 \mathrm{q})$, was die Seltenheit dieser Anomalie bei MDS widerspiegelt. Etwas häufiger findet sich die $\operatorname{del}(9 q)$ in der Literatur dagegen bei der akuten myeloischen Leukämie - hier gehäuft in Kombination mit der Translokation (8;21). Peniket et al. (2005) beschreiben 81 Patienten aus einer Kohorte von mehr als 5000 AML-Patienten mit $\operatorname{del}(9 \mathrm{q})$, von denen 21 Patienten diese als Einzelanomalie aufwiesen, 29 Patienten eine zusätzliche $t(8 ; 21)$ hatten und 31 Patienten andere zusätzliche zytogenetische Veränderungen aufwiesen.

Die 1996 von Schoch et al. anhand von 51 Patienten beschriebene ungünstige Prognose der AML bei Kombination von $\mathrm{t}(8 ; 21)$ und zusätzlicher $\operatorname{del}(9 \mathrm{q})(\mathrm{n}=14)$ mit einer medianen Überlebenszeit von nur 12,5 Monaten konnte anhand der größeren Fallzahl von Peniket et al. (2005) nicht nachgewiesen werden. Hier betrug das 5-Jahres-Überleben im Fall der Kombination aus $\mathrm{t}(8 ; 21)$ und $\operatorname{del}(9 \mathrm{q})$ sogar $75 \%$ und $45 \%$ bei Patienten mit del $(9 \mathrm{q})$ als Einzelanomalie. Damit waren beide Gruppen besser als die Kontrollgruppe mit normalem Karyotyp und einer 5-Jahres-Überlebensrate von nur 35\%. Allerdings wurden hier AML-Patienten hinsichtlich ihrer Prognose untersucht, was nicht vergleichbar mit unseren Daten primärer MDS-Patienten ist. Interessanterweise beschreiben Peniket et al. (2005) bei den Patienten mit del(9q) eine signifikant höhere Rate an Dysplasiezeichen (74\% Dysplasiezeichen der erythrozytären Vorstufen, sowie $90 \%$ pathologische Vakuolisierung bei den myelozytären Vorläufern).

Als Pathomechanismus der AML mit del(9q) wird von Sweetser et al. (2005) der Verlust von Tumorsuppressorgenen in der deletierten Region beschrieben, durch den die Aktivität von sieben von elf Genen vermindert ist und in einer vermehrten Proliferation und verlängerten Überlebenszeit der klonalen Zellen resultiert. Diese letztgenannten Mechanismen sind auch bei MDS als Pathomechanismus beschrieben (Bejar et al. 2011), der letztlich die Entstehung einer AML aus MDS begünstigen könnte. Die hohe Rate an Dysplasiezeichen, zusammen mit der Seltenheit der del(9q) bei MDS und der hohen HR von 3,8 für die AML-freie Überlebenszeit dieser Gruppe spricht hier eventuell für eine häufig sekundäre Genese dieser AML. Dazu würden dann aber die besseren Überlebenszeiten der Untersuchung von Peniket et al. (2005) dieser Patienten nicht passen, da sekundäre AML normalerweise eine schlechtere Prognose als de-novo-AML- Erkrankungen aufweisen.

Zusammenfassend lässt sich anhand der vorgelegten Daten aber mit ausreichender statistischer Sicherheit schließen, dass die Prognose von Patienten mit Monosomie 9 schlechter ist, als die der Vergleichsgruppe und dass vor allem das Risiko der Entwicklung einer AML stark erhöht ist. 


\subsubsection{Veränderungen am Chromosom 11}

Hinsichtlich der Überlebenszeit zeigt sich bei den in der Gruppe ANY 11 gebündelten Veränderungen am Chromosom 11 eine Tendenz zu besserem Überleben mit einer HR von 0,6 $(\mathrm{p}=0,311)$. Bei den acht Patienten in dieser Gruppe lag die mediane ÜLZ mit 47,5 Monaten und die AML-freie Überlebenszeit bei sieben Patienten mit 65,8 Monaten deutlich über denen der Arbeiten von Pozdnyakova et al. (2008) mit 13,5 Monaten für das mediane Überleben von vier Patienten mit Trisomie 11 und Schanz et al. (2012) von 11 Monaten für jeweils die mediane ÜLZ und das AFS bei fünf Patienten. Da in unseren Berechnungen auch Patienten mit anderen Veränderungen subsumiert waren als der Trisomie 11, und die Gruppe mit nur acht respektive sieben Patienten weiterhin nur klein ist, ist die Vergleichbarkeit sowie die Aussagekraft der Berechnungen sehr kritisch zu bewerten und stark eingeschränkt.

\subsubsection{Monosomie 12, 14 und 18}

Für die Gruppen mit totaler oder partieller Monosomie der Chromosomen 12, 14 und 18 fällt gemeinsam eine Tendenz zu einer verkürzten Überlebenszeit auf. Die HR liegt jeweils zwischen 1,2 und 1,7. Das Signifikanzniveau wird hier jedoch jeweils nicht erreicht. Hinsichtlich der partiellen oder totalen Monosomie 12 ist die gute Prognose der del(12p) bereits in mehreren Studien beschrieben (Solé et al. 2005; Haase et al. 2007; Schanz et al. 2012) und hat zur Einordnung dieser Anomalie in die gute Prognosegruppe des IPSS-R geführt (Greenberg et al. 2012). Deshalb war sie in der vorliegenden Analyse auch nicht mehr in dieser Subgruppe inkludiert. Diese gute Prognose scheint aufgrund der Ergebnisse der vorliegenden Arbeit für andere Deletionen am Chromosom 12 so nicht übertragbar zu sein. Haase (2008) beschreibt dann auch z. B. für die Monosomie 12 in einem Kollektiv von 1080 Patienten mit aberrantem Karyotyp die Monosomie 12 in 92,3\% der Fälle ( $\mathrm{n}=24)$ als Teil komplexer Anomalien. Diese Daten sind jedoch nicht direkt vergleichbar, und bei fehlender statistischer Relevanz ist diese Frage hier nicht abschließend zu klären.

Für Monosomien am Chromosom 14 konnten keine Voruntersuchungen in der Literatur gefunden werden. Bei der sehr kleinen Gruppe von nur fünf Patienten und schlechter statistischer Aussagekraft bleibt die Prognose dieser seltenen Anomalie weiterhin unbestimmt. Am ausgeprägtesten ist die Tendenz hin zu verkürzter Überlebenszeit bei der Monosomie 18 mit einer HR von 1,7 ( $\mathrm{p}=0,275)$. Hier zeigt sich auch in der Analyse des AML-freien Überlebens die HR mit 2,9 ( $\mathrm{P}=0,175)$ deutlich erhöht. Da partielle oder totale Monosomien am Chromosom 18 fast ausschließlich als Teil eines komplex-aberranten Karyotyps beobachtet werden (93,6\% von insgesamt 78 Fällen) (Haase 2008), wäre hier analog der Monosomie 
5 zu diskutieren, ob in unserer Untersuchung eventuell kryptische Anomalien in der klassischen Chromosomenbänderungsanalyse übersehen wurden und hier nicht vielleicht eher prognostisch ungünstigere Mehrfachanomalien vorlagen, wie für die Monosomie 5 beschrieben (Zemanova et al. 2014). Zu diskutieren wäre auch, ob der Verlust von genetischem Material am Chromosom 18 ebenfalls zu genetischer Instabilität führt und somit eine raschere klonale Evolution dieser Erkrankungen erfolgt. Um dies zu beweisen, wären große Studien mit wiederholten zytogenetischen Untersuchungen im Verlauf der Erkrankung notwendig, die aufgrund des seltenen Vorkommens der totalen oder partiellen Monosomie 18 tausende Patienten erfassen müssten. Denn selbst in der Untersuchung von Haase et al. (2007) an 2072 Patienten trat die -18/del(18q) zwar bei 7\% der untersuchten Patienten auf, davon aber nur bei drei Patienten als isolierte Veränderung und bei zwei Patienten mit nur einer weiteren Anomalie. Die einzige andere Erwähnung der Monosomie 18 findet sich in der medizinischen Dissertation von Schanz (2003), in der den Deletionen am Chromosom 18 bei schlechter statistischer Verwertbarkeit und keiner gesonderten Analyse der Anomalie bei isoliertem Auftreten, eine mittlere Überlebenszeit von 3,6 Monaten attestiert wir.

Somit lassen sich aus den vorliegenden Daten keine verlässlichen Schlussfolgerungen für die Prognose von Patienten mit Einzelanomalien am Chromosom 12, 14 und 18 beim myelodysplastischen Syndrom ziehen.

\subsubsection{Monosomie (13q)}

In der vorliegenden Arbeit weisen MDS-Patienten mit Deletion (13q) eine sehr gute Prognose auf. Die mittlere Überlebenszeit konnte mit 111,1 Monaten errechnet werden, was sich auch in der multivariaten Analyse mit einer HR von 0,3 ( $\mathrm{p}=0,015)$ als hochsignifikant besser gegenüber der Kontrollgruppe darstellt. Im Beobachtungszeitraum traten dann auch in dieser Subgruppe keine Fälle einer AML auf. Damit würde die Deletion (13q) in die zytogenetische Gruppe des very good risk nach IPSS-R gehören und nicht in die Gruppe mit intermedärem Risiko. Auch in der Arbeit von Schanz et al. (2012) werden von den Patienten mit Monosomie 13 und Deletion (13q) medianes Überleben und AML-freies Überleben nicht erreicht. Da in dieser Arbeit Patienten mit Monosomie 13 und del(13q) zusammengefasst wurden, ist eine direkte Vergleichbarkeit allerdings nicht gegeben.

Die Ergebnisse beider Arbeiten werden auch durch die Untersuchung von Hosokawa et al. (2012) gestützt, der 22 Patienten mit Knochenmarkversagen genetisch untersuchte und bei 16 dieser Patienten eine del(13q) nachweisen konnte, in sechs Fällen davon mit ergänzenden Aberrationen. Alle Patienten waren aufgrund fehlender Dysplasiezeichen im Knochenmark als unklassifierbares MDS diagnostiziert worden. Bemerkenswerterweise ließ sich auch bei 
19 Patienten der Kohorte ein Paroxysmale-nächtliche-Hämoglobinurie (PNH)-Klon nachweisen und die Patienten sprachen alle gut auf eine immunsuppressive Therapie an. Die 10Jahres-Überlebensrate betrug $83 \%$ bei den Patienten mit del(13q) alleine und $67 \%$ bei den Patienten mit zusätzlichen Aberrationen (Hosokawa et al. 2012).

Nun wäre es aufgrund der fehlenden Dysplasiezeichen im Knochenmark natürlich möglich, dass bei den hier untersuchten Patienten aufgrund des ungewöhnlich guten Überlebens überhaupt kein MDS vorlag, sondern möglicherweise eine andere hämatologische Erkrankung, die sich ebenfalls mit peripheren Zytopenien manifestiert. Dieser These gehen Holbro et al. (2013) in ihrer Analyse an 86 eigenen Patienten nach, die hier in Abwesenheit von Dysplasiezeichen als aplastische Anämie klassifiziert worden waren und für die eine zytogenetische Untersuchung verfügbar war. Bei insgesamt sechs Patienten der Kohorte (7\%) war eine Deletion (13q) nachweisbar, und die Überlebensdaten dieser Patienten waren mit denen von Hosokawa et al. (2012) vergleichbar (Holbro et al. 2013). In der vorliegenden Arbeit konnten insgesamt 18 Patienten mit Deletion (13q) untersucht werden. Dabei lagen nach FAB/WHO-Klassifikation zwei Fälle einer RAEB vor, viermal die Diagnose RARS, siebenmal RA, zwei Fälle einer CMML sowie zweimal REAB-T und ein Fall eines MDS-U. Diese Verteilung auf verschiedenen Subtypen mit unterschiedlichen morphologischen Besonderheiten und auch erhöhtem Blastenanteil im Knochenmark spricht gegen die Theorie, das gute Überleben sei eventuell einzig durch die Klassifizierung von Patienten mit Aplastischer Anämie als unklassifizierbares MDS zu erklären.

In jedem Fall sollte bei Patienten mit Knochenmarksversagen und Nachweis einer Deletion (13q) in Abwesenheit von Dysplasiezeichen der Hämatopoese die Diagnose MDS kritisch hinterfragt werden und die Aplastische Anämie als Differentialdiagnose diskutiert werden. Im Einzelfall kann eventuell eine FISH-Analyse die Diagnose eingrenzen, und eine immunsuppressive Therapie sollte erfolgen (Hosokawa et al. 2012; Holbro et al. 2013). Die hier vorgelegten Daten belegen, dass die Prognose von Patienten mit MDS und isolierter Deletion (13q) zweifelsfrei deutlich besser ist als die der Patienten mit intermediärem zytogenetischem Risiko.

\subsubsection{Monosomie 16}

Die HR für das Gesamtüberleben und das AML-freie Überleben sind in Ermangelung von Ereignissen bei immerhin acht Patienten nicht berechenbar. Damit ist die Prognose sicher vergleichbar, wenn nicht besser als bei den Patienten der Kontrollgruppe und die Einordnung dieser Veränderung in die Gruppe des intermediate risk/good risk steht demzufolge nicht in Frage. 


\subsubsection{Veränderungen am Chromosom 20}

Im Gegensatz dazu hat die sehr heterogene Gruppe verschiedenster Anomalien am Chromosom 20 in unserer Untersuchung eine deutlich schlechtere Prognose. Dies gilt sowohl für die mediane Überlebenszeit mit nur 10,8 Monaten in der univariaten Analyse und mit einer HR von 2,1 ( $\mathrm{p}=0,117)$ als auch für das deutlich verkürzte mittlere AML-freie Überleben mit nur 18,9 Monaten und einer HR von 4,2 ( $\mathrm{p}=0,057)$. In dieser Gruppe waren einzig die del(20q) ausgenommen, die zu den häufigeren Veränderungen bei myelodysplastischen Syndromen zählt und deren Prognose deshalb bereits bekannt ist. Nach IPSS-R wird die del(20q) in die Gruppe des good risk eingeordnet. Die aktuell untersuchten Veränderungen am Chromosom 20 wären demgegenüber prognostisch eher dem poor risk bzw. dem very poor risk nach IPSS-R zuzuordnen.

In der Publikation von Haase (2008) wird z.B. die totale Monosomie 20 ausschließlich als Teil eines komplex-aberranten Karyotyps beobachtet und kommt als isolierte Veränderung bei 22 Fällen nicht vor (Haase 2008). Da es bei den hier untersuchten Fällen um eine sehr heterogene Gruppe verschiedener chromosomaler Veränderungen handelt, lässt sich eine These zur Genese der schlechten Prognose nur schwierig formulieren. Möglich ist auch hier eine resultierende klonale Instabilität mit rascher klonaler Evolution. Auffällig ist in jedem Fall die große Differenz zwischen der guten Prognose der del(20q) und der schlechten Prognose aller anderen Veränderungen des Chromosom 20. Literatur zu diesen seltenen Veränderungen konnte trotz intensiver Suche nicht gefunden werden. Aufgrund dieser neuen Daten sollte die Einordnung von Veränderungen am Chromosom 20 in die zytogenetische Risikogruppe des intermediate risk des IPSS-R nochmals überdacht und eventuell sogar geändert werden.

\subsubsection{Trisomie 21}

Die Trisomie 21 tritt zum einen als konstitutionelle Anomalie auf und wird dann als DownSyndrom bezeichnet. Betroffene Kinder sind etwa 20-fach häufiger von einer AML und sogar 500-fach häufiger von der in der Normalbevölkerung seltenen akuten Megakaryozyten Leukämie betroffen (Lange 2000). 4 - 10\% der mit Down-Syndrom geborenen Kinder haben ein transientes myeloproliferatives Syndrom (TMD) und wiederum $20 \%$ von diesen entwickeln im Kindesalter eine akute Megakaryozyten-Leukämie binnen vier Jahren (Malinge et al. 2009).

Da im Bezug auf maligne Erkrankungen bei Personen mit Down-Syndrom nahezu ausschließlich die Inzidenz hämatologischer Erkrankungen erhöht ist, nicht aber die von soliden 
Tumoren (Hasle 2001), scheint im Falle der konstitutionellen Trisomie 21 ein maligne Transformation der hämatopoetischen Vorläuferzellen begünstigt zu werden (Malinge et al. 2009). Bei den myelodysplastischen Syndromen handelt es sich bei Fällen mit Trisomie 21 in aller Regel um eine erworbene Anomalie, die nur in Teilen der hämatopoetischen Vorläuferzellen auftritt (Mosaik). Diese erworbene Trisomie 21 ist Gegenstand der vorliegenden Untersuchung. Hinsichtlich der Prognose der Trisomie 21 bei MDS beschreiben Wan et al. (1999) fünf Patienten mit Trisomie 21 als Einzelanomalie. Zwei Patienten hatten eine AML und drei ein MDS (2 Fälle mit RAEB und ein Fall mit RAEB-t). Alle drei wiesen eine rapide Krankheitsprogression und signifikante Thrombopenie auf (Wan et al. 1999). Bei der Trisomie 21 handelt es sich in Kombination mit anderen Anomalien durchaus um eine der häufigeren Anomalien bei MDS. Sie war in einem Kollektiv von 1187 Patienten mit unbehandelter AML und MDS in 3,3\% $(\mathrm{n}=37)$ nachweisbar. Allerdings nur in $0,4 \%(\mathrm{n}=4)$ als Einzelanomalie (Cortes et al. 1995).

Das deckt sich mit den großen Prognosestudien bei MDS der letzten 15 Jahre. Hier werden Häufigkeiten der Trisomie 21 als Einzelanomalie bei ausschließlichen MDS-Patienten zwischen 0,3\% in einem Kollektiv aus 2902 Patienten (Schanz et al. 2012) über 0,46\% (Haase et al. 2007) bis zu 0,8\% im 968 Patienten umfassenden Kollektiv der spanischen Arbeitsgruppe beschrieben (Solé et al. 2005). Bei den entsprechend kleinen Fallzahlen (maximal 13 Patienten) fällt das mediane Überleben dann auch stark different aus. Während Solé et al. (2005) das mediane Überleben bei acht Patienten mit Trisomie 21 als Einzelanomalie mit 14 Monaten angeben $(p=0,02)$, beträgt es in der Arbeit von Schanz et al. (2012) mit neun Patienten 21,5 Monate und in der Studie von Haase et al. (2007) 100,8 Monate innerhalb einer Gruppe von 13 Patienten mit nicht komplexem Karyotyp (d.h. maximal einer weiteren Anomalie). In der Arbeit von Pozdnyakova et al. (2008) hatten die zwei eingeschlossenen Patienten mit Trisomie 21 als Einzelanomalie eine Überlebenszeit von 8 respektive 33 Monaten und in der frühesten Arbeit zur Prognose der Trisomie 21 bei MDS wird bei einer medianen Überlebenszeit von 16 Monaten ebenfalls eine intermediäre Prognose beschrieben (White et al. 1994). Außer in der Untersuchung von Haase et al. (2007) sind die Ergebnisse hinsichtlich der Überlebenszeit ähnlich und würden heute eher der prognostischen Gruppe des poor risk nach IPSS-R entsprechen.

Die früher durchgeführten Untersuchungen bescheinigen also der Trisomie 21 ein eher intermediäres bis schlechtes Überleben, während die Untersuchung von Haase et al (2007) ein gutes bis sehr gutes Überleben nahelegt und die vorliegende Arbeit ein intermediär bis gutes Überleben. In der vorliegenden Arbeit konnte für elf Patienten die mittlere Überlebenszeit 
mit 66,3 Monaten in der univariaten Analyse errechnet werden, nach multivariater Adjustierung ergibt sich eine $\mathrm{HR}$ von $0,9(\mathrm{p}=0,838)$ und kein statistisch relevanter Unterschied zur Kontrollgruppe mit normalem Karyotyp (47,4 Monaten), sodass diese Frage nicht abschlieBend beantwortet werden kann.

Auffällig ist allerdings die HR von 2,3 ( $\mathrm{p}=0,184)$ für das AML-freie Überleben in unserer Untersuchung bei einem mittleren AFS von 83 Monaten. Dies deckt sich mit den Daten von Schanz et al. (2012), wo das mediane AFS mit 100,7 Monaten angegeben wird und damit nur halb so lang ist, wie das mediane AFS anderer Patienten mit Einzelanomalien in dieser Untersuchung mit 203,2 Monaten (Schanz et al. 2012). Diese Zahlen stützten die These von Malinge et al. (2009), die der Trisomie 21 im Falle der konstitutionellen Veränderung die Begünstigung der malignen Transformation hämatopoetischer Vorläuferzellen bescheinigt, was im Umkehrschluss bei MDS mit Trisomie 21 zur kürzeren AML-freien Überlebenszeit beitragen könnte. Solange hier aber keine vergleichenden Genexpressionsanalysen erfolgen, die ähnliche genetische Aktivität bei MDS und konstitutioneller Trisomie 21 in den fraglichen Regionen nachweisen, muss auch dies eine unbewiesenen These bleiben.

\subsubsection{Monosomie X}

Über den Verlust einzelner Gonosomen schließlich ist bereits manches bekannt. Die Inzidenz des Y- Verlustes steigt dabei mit dem Alter an (Ganster et al. 2015) und wird mit einer erhöhten Morbidität und Mortalität sowohl für hämatologische als auch andere Tumoren in Verbindung gebracht (Forsberg et al. 2014). Ähnlich dem Verlust des Y-Chromosoms tritt auch der X-Verlust bei der Frau mit zunehmendem Alter häufiger auf, ist aber insgesamt seltener. Machiela et al. (2015) beschreiben die Prävalenz des X-Chromosom-Verlustes in einer gepoolten Analyse an über 39.0000 Proben von tumorerkrankten und gesunden Frauen mit einer Frequenz von $0,11 \%$ bei Frauen unter 50 Jahren und mit 0,45\% bei Frauen im Alter von 75 Jahren und älter. Dabei ist beim X-Chromosom-Verlust, wiederum analog zum Y-Chromosom-Verlust des Mannes, immer nur ein Teil der hämatologischen Zellen betroffen (Mosaik) (Machiela et al. 2016). Somit fehlen bei Monosomie X, die als konstitutionelle Anomalie ein Turner-Syndrom definiert, analog zur Trisomie 21 in aller Regel entsprechende phänotypische Veränderungen. Umgekehrt sind hämatologische Erkrankungen bei Patienten mit Turner-Syndrom, anders als bei der Trisomie 21, nicht häufiger beschrieben als in der Normalbevölkerung (Manola et al. 2008). Demgegenüber scheinen aber Patienten mit einem erworbenen X-Chromosom-Verlust eine erhöhte Inzidenz an hämatologischen Neoplasien aufzuweisen (Machiela et al. 2016). Während der Verlust des Y-Chromosoms zu den häufigeren Veränderungen bei myelodysplastischen Syndromen zählt und mit einer guten 
Prognose nach IPSS (Greenberg et al. 1997) und einer sehr guten Prognose nach IPSS-R (Greenberg et al. 2012) assoziiert ist, ist die Häufigkeit des X-Chromosom-Verlustes mit 0,20,3\% als Einzelanomalie und mit bis zu 1,5\% in Kombination mit anderen Anomalien bei MDS und AML beschrieben (Abruzzese et al. 1997; Zeidan und Phatak 2008; Schanz et al. 2012); die Prognose ist jedoch noch nicht sicher evaluiert.

Haase et al. (2007) konnten für sechs Patienten in einem Kollektiv aus 2072 Patienten eine mediane Überlebenszeit von 56,4 Monaten errechnen, was sich mit den medianen Überlebensdaten der vorliegenden Arbeit von 59,1 Monaten deckt. Dieses Ergebnis ließ sich auch in der multivariaten Analyse mit einer HR von 0,2 bestätigen. Das Signifikanzniveau wird dabei nur knapp verfehlt( $\mathrm{p}=0$,052). In der Analyse von Schanz et al. (2012) ergab die Analyse von immerhin neun Patienten dagegen nur eine mediane Überlebenszeit von 16 Monaten und eine mediane AML-freie Überlebenszeit von 14 Monaten und damit ein intermediäres Risiko für die Monosomie X. In unserer Analyse ist das mittlere AFS mit 49,9 Monaten deutlich länger, aber bei nur fünf Patienten in der multivariaten Analyse mit einer HR von 1 $(p=0,975)$ nicht signifikant. Eine ebenfalls ungünstige Prognose für die partielle Monosomie X beschreibt Olshanskaya et al. (2006). Bei drei Patienten in einer Studie mit insgesamt 127 Patienten mit MDS wurde bei Verlust an verschiedenen Bruchstellen des langen Armes eines X-Chromosoms ein ungünstiger Verlauf beobachtet (Olshanskaya et al. 2006).

Aufgrund unserer neuen Daten sollte die Einstufung der Monosomie X in die Gruppe des intermediären Risikos nach IPSS-R nochmals überdacht und gegebenenfalls in good risk geändert werden.

\subsubsection{Balancierte Translokationen}

In der Gruppe mit balancierten Translokationen in unserer Untersuchung kommen nahezu alle Chromosomen, einschließlich der Geschlechtschromosomen, als Translokationspartner vor. Diese Gruppe ist mit 44 Fällen die zweitgrößte dieser Untersuchung, trotzdem kommen die meisten einzelnen Translokationen nur ein- bis maximal viermal vor. Dies passt zu den Ergebnissen von Nomdedeu et al. (2016), dass Translokationen bei MDS und CMML insgesamt selten sind. Die Kollegen konnten in einem Kollektiv von 1653 Patienten mit abnormalem Karyotyp nur 168 Patienten mit Translokationen sowohl als Einzel-, wie auch Doppeltranslokationen als auch in komplex-aberranten Konstellationen identifizieren. In der multivariaten Analyse konnten sie kein signifikant schlechteres medianes Überleben als in ihrer Kontrollgruppe zeigen, welches sich in der Gruppe des intermediate risk nach IPSS-R bewegt. Die Kollegen regen deshalb an, Einzel- und Doppeltranslokationen außer der t(3q), deren schlechte Prognose bereits evaluiert und im IPSS-R integriert ist, in der Gruppe des 
intermediate risk nach IPSS-R zu belassen (Nomdedeu et al. 2016). Die Kontrollgruppe war hier definiert als abnormaler Karyotyp ohne das Auftreten von Translokationen und nicht wie in unserer Untersuchung ein normaler Karyotyp ohne Aberrationen.

Eine schlechte Prognose für Patienten mit Translokationen bei MDS mit verkürzter Überlebenszeit fanden Suh et al. (2016). In der koreanischen Studie wurden 40 sowohl behandelte als auch unbehandelte Patienten mit MDS, mit balancierten und unbalancierten Translokationen zusammengefasst. 75\% $(\mathrm{n}=30)$ von ihnen hatten einen komplex-aberranten Karyotyp (Suh et al., 2016). Somit ist eine Vergleichbarkeit beider Studien hinsichtlich der Prognosedaten mit den hier vorgelegten Daten einer Subgruppe mit ausschließlich balancierten Translokationen als Einzelanomalie bei primären MDS nicht sinnvoll.

In der Untersuchung von Haase et al. (2007) werden einige wenige Einzeltranslokationen gesondert betrachtet. Die Häufigkeit in einem Kollektiv von 2072 Patienten wird für die $t(11 q), t(7 q), t(1 q), t(15 q)$, jeweils mit 0,4-0,6\% angegeben. Diese werden dort anhand ihres medianen Überlebens von 32,1 Monaten für die $\mathrm{t}(11 \mathrm{q}), 34,7$ Monaten für die $\mathrm{t}(7 \mathrm{q})$ und die $\mathrm{t}(1 \mathrm{q})$, und nicht erreichtem medianen Überleben für die $\mathrm{t}(15 \mathrm{q})$ in die prognostisch günstige zytogenetische Gruppe eingeordnet. Eine direkte Vergleichbarkeit zu der vorliegenden Analyse ist somit wiederum nicht gegeben.

In der hier vorliegenden Untersuchung zeigt sich nun bei Patienten mit einer einzelnen Translokation ein medianes Überleben von 50,7 Monaten, und das AFS wird nicht erreicht. In der multivariaten Analyse ergibt sich dann auch eine HR von 0,6, die hinsichtlich des Überlebens auch statistisch signifikant ist $(\mathrm{p}=0,046)$. Hinsichtlich des AFS zeigt sich jedoch in der multivariaten Analyse nur ein Trend hin zur besseren Prognose mit einer HR von 0,65 $(\mathrm{p}=0,381)$. Eine Verschiebung von balancierten Translokationen als Einzelanomalie bei MDS aus der Gruppe des intermediären Risikos in die Gruppe mit gutem Risiko wäre anhand dieser neuen Daten zu diskutieren. Eine Analyse der gepoolten Daten aller balancierten Translokationen aus dem Kollektiv der Arbeit von Haase et al. (2007) könnte darüber hinaus bei größerer Fallzahl interessant sein.

\subsubsection{Markerchromosomen}

Markerchromosomen als distinkte zytogenetische Gruppe wurden erstmals in der Analyse von Schanz et al. (2012) untersucht. Hier konnte für eine Gruppe aus fünf Patienten ein medianes Überleben von 20,7 Monaten errechnet werden. Das mittlere AML-freie Überleben wurde nicht erreicht. In der vorliegenden Untersuchung zeigt sich die mediane Überlebenszeit mit 51,5 Monaten eher der guten zytogenetischen Prognosegruppe nach IPSS-R zugehörig. Allerdings wird bei einer HR von 0,8 und einem p-Wert von 0,497 kein statistisch 
signifikantes Ergebnis bei den elf Patienten dieser Gruppe erreicht. Dies gilt auch für die AML-freie Überlebenszeit mit 139,9 Monaten in der univariaten Analyse, die mit einer HR von 0,5 aber auch ebenfalls das Signifikanzniveau verfehlt $(p=0,523)$. Innerhalb dieser Gruppe resultiert aus der Markerchromosomenbildung offenbar keine klonale Instabilität, wie wir sie für die Veränderungen am Chromosom 20, die Monosomie 18 und die Monosomie 5 diskutiert haben, obwohl auch Markerchromosomen häufig innerhalb komplex-aberranter Veränderungen vorkommen (Haase et al. 2007).

\subsubsection{Einzelchromosomaberrationen mit einer absoluten Häufigkeit von $<5$ Fällen}

Da sich über die Prognose einzelner Anomalien dieser Gruppe aufgrund der Seltenheit ihres Auftretens keinerlei Aussage treffen läßt, zeigt sich nach gesonderter Analyse der oben beschriebenen Subgruppen eine mediane Überlebenszeit von 51,3 Monaten in der univariaten Anlyse mit einer HR von 0,7 ( $\mathrm{p}=0,172)$. Damit besteht gegenüber der Kontrollgruppe kein signifikanter Unterschied, und gegenüber der Analyse von Schanz et al. (2012) liegt die mediane Überlebenszeit etwa doppelt so hoch (23,3 Monate). Dies spricht dafür, dass die Gruppe der verbliebenen Anomalien mindestens der Gruppe des intermediate risk nach IPSS$\mathrm{R}$ zuzuordnen sind.

\subsection{Methodische Kritik}

Bei retrospektiven Analysen stellt sich das Problem der lückenlosen Datenerhebung. Bei insgesamt nur 382 Fällen mit Einzelchromosomenaberrationen in einer Datenbank mit mehr als 7000 Fällen primärer unbehandelter myelodysplastischer Syndrome standen leider für die statistische Analyse nur für rund drei Viertel der Patienten klinische Daten zur Verfügung. Obwohl also bei den absoluten Häufigkeiten noch Subgruppen von acht Patienten das Minimum bilden, bedeutete dies für die univariate Analyse bereits mitunter nur noch Gruppengrößen von 5 Patienten und entsprechend große 95\%-Konfidenzintervalle mit der daraus resultierenden statistischen Ungenauigkeit. Noch ausgeprägter ist dieser Effekt dann in der multivariaten Untersuchung, sodass nur für wenige Subgruppen eine prognostische Einschätzung mit ausreichender statistischer Sicherheit zu erzielen war.

Trotzdem wurden Signifikanzniveaus für verschiedene Subgruppen erreicht und das Ziel der Arbeit, nämlich die Gruppe distinkter Anomalien mit unbestimmter Prognose in der Gruppe des intermediären Risikos des IPSS-R weiter zu verkleinern, konnte erreicht werden.

\subsection{Ausblick}

Da es eine noch größere Datenbank mit mehr Fällen unbehandelter primärer MDS-Patienten im Zeitalter neuer Therapien und nach dem Ende des therapeutischen Nihilismus bei mye- 
lodysplastischen Syndromen zur weiteren Spezifizierung distinkter Einzelchromosomenanomalien voraussichtlich nicht geben wird, wäre es zur Klärung der offenen Fragen dieser Arbeit sicher hilfreich, bei den Einzelanomalien mit eingeschränktem Gesamtüberleben oder verkürztem AML-freien Überleben mittels ergänzender Untersuchungen (z. B. Fluoreszenzin-situ-Hybrisisierung) gezielt nach kryptischen Anomalien zu suchen oder mittels sequenzieller Knochenmarkuntersuchungen klonale Instabilität nachzuweisen (z. B. bei der Monosomie 5, 9, 18 und Veränderungen am Chromosom 20).

Für die Anomalien mit statistisch nachgewiesenem besseren medianen Überleben (Deletion (13q), Monosomie X und balancierte Translokationen, sowie für die Subgruppen mit nachgewiesener oder hochwahrscheinlich verkürzter AML-freier Überlebenszeit (Monosomie 9, Veränderungen am Chromosom 20, Monosomie 5), sollte überprüft werden, ob sich die Ergebnisse im IPSS-R ebenfalls als valide erweisen.

Künftig wird die molekulare Analyse mutierter Gene für die prognostische Klassifikation der myelodysplastischen Syndrome sicher zunehmend an Relevanz gewinnen. Für einzelne häufigere Veränderungen kann bereits heute eine Prognoseabschätzung erfolgen. Die Integration dieser zusätzlichen Informationen in die bestehenden prognostischen Scoringsysteme fällt aufgrund der wiederum hohen Heterogenität der genetischen Veränderungen bei MDS analog der Zytogenetik aber derzeit noch schwer. 


\section{$5 \quad$ Zusammenfassung}

Myelodysplastische Syndrome sind eine heterogene Erkrankungsgruppe der hämatopoetischen Vorläuferzellen, die in einer ineffektiven Hämatopoese resultieren und sich mit verschiedenen Zytopenien im peripheren Blut manifestieren. Symptome wie Leistungsminderung, Schwäche, Infektionen und Blutungen führen mit Hilfe der Untersuchung von Blutausstrich und Knochenmarkszytologie zur Diagnose.

Die Zytogenetik ist ein gut validierter Prognosefaktor, der im Rahmen des IPSS-R für gut 90\% der Patienten bei Diagnosestellung eine Einschätzung der Überlebenszeit und AMLfreien Überlebenszeit erlaubt, und damit eine individuelle Therapieplanung ermöglicht.

Myelodysplastische Syndrome sind hinsichtlich ihrer Zytogenetik höchst variabel und auch nach Spezifizierung der zytogenetischen Risikogruppen im IPSS-R ist bis heute die Prognose fast jeder zehnten Einzelchromosomanomalie bei MDS weiterhin unbestimmt.

Die vorliegende Arbeit untersucht deshalb die Häufigkeit distinkter Einzelchromosomenanomalien bei primären unbehandelten MDS in der bisher größten Datenbank von über 7000 Fällen und ihre prognostische Bedeutung hinsichtlich des Gesamtüberlebens und der AMLfreien Überlebenszeit mittels uni- und multivariater Analyse.

Wesentliche Ergebnisse zeigen sich in der multivariaten Untersuchung im Hinblick auf die Deletion (13q) und bei balancierten Translokationen. Hier ist das Gesamtüberleben signifikant besser als in der Kontrollgruppe mit normalem Karyotyp. Grenzwertig besser mit einem p-Wert von 0,52 ist auch die Gruppe der Patienten mit Monosomie X. Eine Veränderte Einornung in die zytogenetische Risikogruppe des good risk nach IPSS-R ist im Weiteren zu diskutieren. Patienten mit Veränderungen am Chromosom 20 exklusive der del(20q) haben mit einer HR von 2,1 $(\mathrm{p}=0,117)$ und Patienten mit Monosomie 9 mit einer HR von 1,9 $(p=0,071)$ ein kürzeres Gesamtüberleben im Verhältnis zur Kontrollgruppe.

Hinsichtlich der AML-freien Überlebenszeit ist diese für die Patienten mit Monosomie 9 mit einer HR von 3,8 $(\mathrm{p}=0,013)$ signifikant verkürzt, und für Patienten mit Veränderungen am Chromosom 20 wird die Signifikanz bei einer HR von 4,2 und $p=0,057$ nur gering verfehlt. Patienten mit partieller oder totaler Monosomie 5 und 18 haben mit einer HR von 3,3 $(\mathrm{p}=0,105)$ respektive $2,9(\mathrm{p}=0,175)$ ebenfalls ein deutlich erhöhtes Risiko für ein verkürztes AML-freies Überleben. Die Einordnung in die zytogenetische Risikogruppe des poor risk nach IPSS-R ist auch hier zu diskutieren.

Somit konnte die vorliegende Arbeit für die zytogenetischen Subgruppen der Deletion (13q), der balancierten Translokationen, der Monosomie X, der Veränderungen am Chromosom 20 und der Monosomie 9 die Prognose hinsichtlich des Gesamtüberlebens spezifizieren sowie für die Monosomie 9, für Veränderungen am Chromosom 20 sowie der Monosomie 5 und Monosomie18 hinsichtlich der AML-freien Überlebenszeit. 


\section{Literaturverzeichnis}

Abruzzese E, Rao PN, Slatkoff M, Cruz J, Powell BL, Jackle B, Pettenati MJ (1997): Monosomy X as a recurring sole cytogenetic abnormality associated with myelodysplastic diseases. Cancer Genet Cytogenet $\underline{\text { 33, }} 140$ 146

Alfaro R, Pérez-Granero A, Durán MA, Besalduch J, Rosell J, Bernués M (2008): dup(1)(q21q32) as a sole cytogenetic event is associated to a leukemic transformation in myelodysplastic syndromes. Leuk Res $\underline{32}$, 159-161

Arber DA, Orazi A, Hasserjian R, Thiele J, Borowitz MJ, Le Beau MM, Bloomfield CD, Cazzola M, Vardiman JW (2016): The 2016 revision to the World Health Organization classification of myeloid neoplasms and acute leukemia. Blood 127, 2391-2405

Aul C, Gattermann N, Schneider W (1992): Age-related incidence and other epidemiological aspects of myelodysplastic syndromes. Br J Haematol $\underline{82}, 358-367$

Bacher U, Schanz J, Braulke F, Haase D (2015): Rare cytogenetic abnormalities in myelodysplastic syndromes. Mediterr J Hematol Infect Dis $\underline{7}$, e2015034

Baldazzi C, Luatti S, Zuffa E, Papayannidis C, Ottaviani E, Marzocchi G, Ameli G, Bardi MA, Bonaldi L, Paolini R et al. (2016): Complex chromosomal rearrangements leading to MECOM overexpression are recurrent in myeloid malignancies with various 3q abnormalities. Genes Chromosomes Cancer $\underline{55}$, 375-388

Bejar R, Levine R, Ebert BL (2011): Unraveling the Molecular Pathophysiology of Myelodysplastic Syndromes. J Clin Oncol 29, 504-515

Bellamy WT (2001): Vascular endothelial cell growth factor is an autocrine promoter of abnormal localized immature myeloid precursors and leukemia progenitor formation in myelodysplastic syndromes. Blood $\underline{97}$, $1427-1434$

Bennett JM, Catovsky D, Daniel MT, Flandrin G, Galton DA, Gralnick HR, Sultan C (1982): Proposals for the classification of the myelodysplastic syndromes. Br J Haematol $\underline{51}$, 189-199

Collado R, Badía L, García S, Sánchez H, Prieto F, Carbonell F (1999): Chromosome 11 abnormalities in myelodysplastic syndromes. Cancer Genet Cytogenet 114, 58-61

Cortes JE, Kantarjian H, O’Brien S, Keating M, Pierce S, Freireich EJ, Estey E (1995): Clinical and prognostic significance of trisomy 21 in adult patients with acute myelogenous leukemia and myelodysplastic syndromes. Leukemia $\underline{9}, 115-117$

Cox DR (1972): Regression models and life tables. JR Stat Soc Series B Methodol $\underline{34}$ (2),187-200

De Braekeleer M, Le Bris M-J, De Braekeleer E, Basinko A, Morel F, Douet-Guilbert N (2015): 3q26/EVI1 rearrangements in myeloid hemopathies: a cytogenetic review. Future Oncol 11, 1675-1686

De Souza Fernandez T, Omellas MH, Otero de Carvalho L, Tabak D, Abdelhay E (2000): Chromosomal alterations associated with evolution from myelodysplastic syndrome to acute myeloid leukemia. Leuk Res $\underline{24}$, 839-48

Doll DC, List AF (1989): Myelodysplastic syndromes. West J Med 151, 161-167

Ferdowsi S, Shirkoohi R, Toogeh G (2013): Myelodysplastic Syndrome with 6q Deletion as the Sole Chromosome Abnormality in an Iranian Patient: A Case Report with Review of Literature. Iran J Public Health $\underline{42}$, 1187-1191

Fisher WB, Armentrout SA, Weisman R, Graham RC (1973): „Preleukemia“. A myelodysplastic syndrome often terminating in acute leukemia. Arch Intern Med 132, 226-232

Fonatsch C, Haase D, Freund M, Bartels H, Tesch H (1991): Partial trisomy 1q. Cancer Genet Cytogenet $\underline{56}$, 243-253 
Forsberg LA, Rasi C, Malmqvist N, Davies H, Pasupulati S, Pakalapati G, Sandgren J, Diaz de Ståhl T, Zaghlool A, Giedraitis V et al. (2014): Mosaic loss of chromosome Y in peripheral blood is associated with shorter survival and higher risk of cancer. Nat Genet $\underline{46}, 624-628$

Ganster C, Kämpfe D, Jung K, Braulke F, Shirneshan K, Machherndl-Spandl S, Suessner S, Bramlage CP, Legler TJ, Koziolek MJ et al. (2015): New data shed light on Y-loss-related pathogenesis in myelodysplastic syndromes: Y-chromosome loss in myelodysplastic syndromes. Genes Chromosomes Cancer 54, 717-724

Germing U, Strupp C, Kundgen A, Bowen D, Aul C, Haas R, Gattermann N (2004): No increase in age-specific incidence of myelodysplastic syndromes. Haematologica $\underline{89}, 905-910$

Gozzetti A, Crupi R, Defina M, Bocchia M, Raspadori D, Lauria F (2009): Isolated deletion of 6q in a patient with myelodysplastic syndrome. Cancer Genet Cytogenet 192, 51

Greenberg PL (1983): The smoldering myeloid leukemic states: clinical and biologic features. Blood $\underline{61}$, 10351044

Greenberg PL, Cox C, LeBeau MM, Fenaux P, Morel P, Sanz G, Sanz M, Vallespi T, Hamblin T, Oscier D et al. (1997): International scoring system for evaluating prognosis in myelodysplastic syndromes. Blood $\underline{89}$, 2079-2088

Greenberg PL, Tuechler H, Schanz J, Sanz G, Garcia-Manero G, Sole F, Bennett JM, Bowen D, Fenaux P, Dreyfus F et al. (2012): Revised International Prognostic Scoring System for Myelodysplastic Syndromes. Blood $\underline{120}, 2454-2465$

Greenberg PL, Stone RM, Al-Kali A, Barta SK, Bejar R, Bennett JM, Carraway H, Castro CMD, Deeg HJ, DeZern AE et al. (2017): Myelodysplastic Syndromes, Version 2.2017, NCCN Clinical Practice Guidelines in Oncology. J Natl Compr Canc Netw $\underline{15}, 60-87$

Haase D (2008): Cytogenetic features in myelodysplastic syndromes. Ann Hematol $\underline{\text { 87, 515-526 }}$

Haase D, Fonatsch C, Freund M, Wörmann B, Bodenstein H, Bartels H, Stollmann-Gibbels B, Lengfelder E (1995): Cytogenetic findings in 179 patients with myelodysplastic syndromes. Ann Hematol 70, 171-187

Haase D, Germing U, Schanz J, Pfeilstocker M, Nosslinger T, Hildebrandt B, Kundgen A, Lubbert M, Kunzmann R, Giagounidis AAN et al. (2007): New insights into the prognostic impact of the karyotype in MDS and correlation with subtypes: evidence from a core dataset of 2124 patients. Blood 110, 4385-4395

Haferlach T, Nagata Y, Grossmann V, Okuno Y, Bacher U, Nagae G, Schnittger S, Sanada M, Kon A, Alpermann $\mathrm{T}$ et al. (2014): Landscape of genetic lesions in 944 patients with myelodysplastic syndromes. Leukemia $\underline{28}, 241-247$

Hasle H (2001): Pattern of malignant disorders in individuals with Down's syndrome. Lancet Oncol 2 , 429_ 436

Heim S (1992): Cytogenetic findings in primary and secondary MDS. Leuk Res $\underline{16}, 43-46$

Holbro A, Jotterand M, Passweg JR, Buser A, Tichelli A, Rovó A (2013): Comment to „Favorable outcome of patients who have 13q deletion: a suggestion for revision of the WHO ,MDS-U“ designation“ Haematologica. 2012;97(12):1845-9. Haematologica $\underline{98}$, e46-47

Horiike S, Taniwaki M, Misawa S, Nishigaki H, Okuda T, Yokota S, Kashima K, Inazawa J, Abe T (1990): The unbalanced 1;7 translocation in de novo myelodysplastic syndrome and its clinical implication. Cancer $\underline{65}, 1350-1354$

Hosokawa K, Katagiri T, Sugimori N, Ishiyama K, Sasaki Y, Seiki Y, Sato-Otsubo A, Sanada M, Ogawa S, Nakao S (2012): Favorable outcome of patients who have 13q deletion: a suggestion for revision of the WHO „MDS-U“ designation. Haematologica $\underline{97}, 1845-1849$

Hsiao H-H, Sashida G, Ito Y, Kodama A, Fukutake K, Ohyashiki JH, Ohyashiki K (2006): Additional cytogenetic changes and previous genotoxic exposure predict unfavorable prognosis in myelodysplastic syndromes and acute myeloid leukemia with $\operatorname{der}(1 ; 7)(\mathrm{q} 10 ; \mathrm{p} 10)$. Cancer Genet Cytogenet 165, 161-166 
Jabbour E, Takahashi K, Wang X, Cornelison AM, Abruzzo L, Kadia T, Borthakur G, Estrov Z, O’Brien S, Mallo M et al. (2013): Acquisition of cytogenetic abnormalities in patients with IPSS defined lower-risk myelodysplastic syndrome is associated with poor prognosis and transformation to acute myelogenous leukemia. Am J Hematol 묘, 831-837

Jacobs RH, Cornbleet MA, Vardiman JW, Larson RA, Le Beau MM, Rowley JD (1986): Prognostic implications of morphology and karyotype in primary myelodysplastic syndromes. Blood $\underline{67}, 1765-1772$

Johansson B, Brøndum-Nielsen K, Billström R, Schiødt I, Mitelman F (1997): Translocations between the long arms of chromosomes 1 and 5 in hematologic malignancies are strongly associated with neoplasms of the myeloid lineages. Cancer Genet Cytogenet 99, 97-101

Jotterand Bellomo M, Parlier V, Mühlematter D, Grob JP, Beris P (1992): Three new cases of chromosome 3 rearrangement in bands q21 and q26 with abnormal thrombopoiesis bring further evidence to the existence of a 3q21q26 syndrome. Cancer Genet Cytogenet $\underline{59}, 138-160$

Kantarjian HM, Keating MJ (1987): Therapy-related leukemia and myelodysplastic syndrome. Semin Oncol 14, 435-443

Kantarjian HM, O’Brien S, Ravandi F, Borthakur G, Faderl S, Bueso-Ramos C, Abruzzo L, Pierce S, Shan J, Issa J-P et al. (2009): The heterogeneous prognosis of patients with myelodysplastic syndrome and chromosome 5 abnormalities: How does it relate to the original lenalidomide experience in MDS? Cancer $\underline{115}, 5202$ 5209

Kaplan EL, Meier P (1958): Non-parametric estimation from incomplete observations. J Am Stat Assoc $\underline{53}$, 457-481

Lange B (2000): The management of neoplastic disorders of haematopoiesis in children with Down's syndrome. Br J Haematol 110, 512-524

Lunghi M, Casorzo L, De Paoli L, Riccomagno P, Rossi D, Gaidano G (2010): Derivative

$(1) t(1 ; 16)(p 11 ; p 11.1)$ in myelodysplastic syndrome: a case report and review of the literature. Cancer Genet Cytogenet 196, 89-92

Machiela MJ, Zhou W, Karlins E, Sampson JN, Freedman ND, Yang Q, Hicks B, Dagnall C, Hautman C, Jacobs KB et al. (2016): Female chromosome $\mathrm{X}$ mosaicism is age-related and preferentially affects the inactivated X chromosome. Nature Commun 7, 11843

Malcovati L, Della Porta MG, Strupp C, Ambaglio I, Kuendgen A, Nachtkamp K, Travaglino E, Invernizzi R, Pascutto C, Lazzarino M et al. (2011): Impact of the degree of anemia on the outcome of patients with myelodysplastic syndrome and its integration into the WHO classification-based Prognostic Scoring System (WPSS). Haematologica $\underline{96}, 1433-1440$

Malcovati L, Hellstrom-Lindberg E, Bowen D, Ades L, Cermak J, del Canizo C, Della Porta MG, Fenaux P, Gattermann N, Germing U et al. (2013): Diagnosis and treatment of primary myelodysplastic syndromes in adults: recommendations from the European LeukemiaNet. Blood 122, 2943-2964

Malinge S, Izraeli S, Crispino JD (2009): Insights into the manifestations, outcomes, and mechanisms of leukemogenesis in Down syndrome. Blood 113, 2619-2628

Manola KN, Sambani C, Karakasis D, Kalliakosta G, Harhalakis N, Papaioannou M (2008): Leukemias associated with Turner syndrome: report of three cases and review of the literature. Leuk Res $\underline{32}, 481-486$

Mauritzson N, Albin M, Rylander L, Billström R, Ahlgren T, Mikoczy Z, Björk J, Strömberg U, Nilsson PG, Mitelman F et al. (2002): Pooled analysis of clinical and cytogenetic features in treatment-related and de novo adult acute myeloid leukemia and myelodysplastic syndromes based on a consecutive series of 761 patients analyzed 1976-1993 and on 5098 unselected cases reported in the literature 1974-2001. Leukemia 16, 23662378

Nomdedeu M, Calvo X, Pereira A, Carrió A, Solé F, Luño E, Cervera J, Vallespí T, Muñoz C, Gómez C et al. (2016): Prognostic impact of chromosomal translocations in myelodysplastic syndromes and chronic myelomonocytic leukemia patients. A study by the spanish group of myelodysplastic syndromes: Prognostic Impact of Translocations in MDS. Genes Chromosomes Cancer 55, 322-327 
Nowell PC (1982): Cytogenetics of preleukemia. Cancer Genet Cytogenet $\underline{5}$, 265-278

Olshanskaya YV, Udovichenko AI, Vodinskaya LA, Glasko EN, Parovitchnikova EN, Lorie YY, Dvirnik VN, Savchenko VG, Domracheva EV (2006): Myelodysplastic syndromes with isolated deletion of the long arm of the chromosome X as a sole cytogenetic change. Cancer Genet Cytogenet 167, 47-50

Papaemmanuil E, Gerstung M, Malcovati L, Tauro S, Gundem G, Van Loo P, Yoon CJ, Ellis P, Wedge DC, Pellagatti A et al. (2013): Clinical and biological implications of driver mutations in myelodysplastic syndromes. Blood 122, 3616-3627; quiz 3699

Peniket A, Wainscoat J, Side L, Daly S, Kusec R, Buck G, Wheatley K, Walker H, Chatters S, Harrison C et al. (2005): Del (9q) AML: clinical and cytological characteristics and prognostic implications. Br J Haematol $\underline{129}, 210-220$

Peto R, Pike MC, Armitage P, Breslow NE, Cox DR, Howard SV, Mantel N, McPherson K, Peto J, Smith PG (1977): Design and analysis of randomized clinical trials requiring prolonged observation of each patient. II. analysis and examples. Br J Cancer $\underline{35}, 1-39$

Pozdnyakova O, Miron PM, Tang G, Walter O, Raza A, Woda B, Wang SA (2008): Cytogenetic abnormalities in a series of 1,029 patients with primary myelodysplastic syndromes: a report from the US with a focus on some undefined single chromosomal abnormalities. Cancer 113, 3331-3340

Raskind WH, Tirumali N, Jacobson R, Singer J, Fialkow PJ (1984): Evidence for a multistep pathogenesis of a myelodysplastic syndrome. Blood $\underline{63}, 1318-1323$

Sanada M, Uike N, Ohyashiki K, Ozawa K, Lili W, Hangaishi A, Kanda Y, Chiba S, Kurokawa M, Omine M et al. (2007): Unbalanced translocation $\operatorname{der}(1 ; 7)(q 10 ; p 10)$ defines a unique clinicopathological subgroup of myeloid neoplasms. Leukemia 21, 992-997

Sanz GF, Sanz MA, Vallespí T, Cañizo MC, Torrabadella M, García S, Irriguible D, San Miguel JF (1989): Two regression models and a scoring system for predicting survival and planning treatment in myelodysplastic syndromes: a multivariate analysis of prognostic factors in 370 patients. Blood $\underline{74}$, 395-408

Schanz J: Charakterisierung und prognostische Bedeutung zytogenetischer Befunde bei Myelodysplastischen Syndromen und sekundärer akuter Leukämie nach Myelodysplasie. Med. Diss. Göttingen 2003

Schanz J, Tüchler H, Solé F, Mallo M, Luño E, Cervera J, Granada I, Hildebrandt B, Slovak ML, Ohyashiki K (2012): New Comprehensive Cytogenetic Scoring System for Primary Myelodysplastic Syndromes (MDS) and Oligoblastic Acute Myeloid Leukemia After MDS Derived From an International Database Merge. J Clin On$\operatorname{col} \underline{30}, 820-829$

Schoch C, Haase D, Haferlach T, Gudat H, Büchner T, Freund M, Link H, Lengfelder E, Wandt H, Sauerland MC (1996): Fifty-one patients with acute myeloid leukemia and translocation t(8;21)(q22;q22): an additional deletion in $9 \mathrm{q}$ is an adverse prognostic factor. Leukemia $\underline{10}, 1288-1295$

Shaffer LG, McGowan-Jordan J, Schmid M (Hrsg.): An International System for Human Cytogenetic Nomenclature. Recommendations of the International Standing Committee on Human Cytogenetic Nomenclature. Karger, Basel 2013

Slovak ML, O’Donnell M, Smith DD, Gaal K (2009): Does MDS with der(1;7)(q10;p10) constitute a distinct risk group? A retrospective single institutional analysis of clinical/pathologic features compared to $-7 / \operatorname{del}(7 q)$ MDS. Cancer Genet Cytogenet 193, 78-85

Solé F, Espinet B, Sanz GF, Cervera J, Calasanz MJ, Luño E, Prieto F, Granada I, Hernández JM, Cigudosa JC et al. (2000): Incidence, characterization and prognostic significance of chromosomal abnormalities in 640 patients with primary myelodysplastic syndromes. Grupo Cooperativo Español de Citogenética Hematológica. Br J Haematol 108, 346-356

Solé F, Luno E, Sanzo C, Espinet B, Sanz GF, Cervera J, Calasanz MJ, Cigudosa JC, Milla F, Ribera JM et al. (2005): Identification of novel cytogenetic markers with prognostic significance in a series of 968 patients with primary myelodysplastic syndromes. Haematologica $\underline{90}, 1168-1178$ 
Suh KJ, Cheong J-W, Kim I, Kim H-J, Shin D-Y, Koh Y, Yoon S-S, Min YH, Ahn J-S, Kim Y-K et al. (2016): Prognostic Impact of IPSS-R and Chromosomal Translocations in 751 Korean Patients with Primary Myelodysplastic Syndrome. PLoS ONE $\underline{11}$, e0166245

Sweetser DA, Peniket AJ, Haaland C, Blomberg AA, Zhang Y, Zaidi ST, Dayyani F, Zhao Z, Heerema NA, Boultwood J et al. (2005): Delineation of the minimal commonly deleted segment and identification of candidate tumor-suppressor genes in $\operatorname{del}(9 \mathrm{q})$ acute myeloid leukemia. Genes Chromosomes Cancer 44, 279-291

Toyama K, Ohyashiki K, Yoshida Y, Abe T, Asano S, Hirai H, Hirashima K, Hotta T, Kuramoto A, Kuriya S (1993): Clinical implications of chromosomal abnormalities in 401 patients with myelodysplastic syndromes: a multicentric study in Japan. Leukemia $\underline{7}, 499-508$

Tricot G (1992): Prognostic factors in the myelodysplastic syndromes. Leuk Res $\underline{16}, 109-115$

Tricot G, Boogaerts MA, Wolf-Peeters CD, Berghe H, Verwilghen RL (1985): The myelodysplastic syndromes: different evolution patterns based on sequential morphological and cytogenetic investigations. Br J Haematol 59, 659-670

Tricot G, Mecucci C, Van Den Berghe H (1986): Evolution of the myelodysplastic syndromes. Br J Haematol $\underline{63}, 609-614$

Tsimberidou AM, Estey E, Wen S, Pierce S, Kantarjian H, Albitar M, Kurzrock R (2008): The prognostic significance of cytokine levels in newly diagnosed acute myeloid leukemia and high-risk myelodysplastic syndromes. Cancer $\underline{113}, 1605-1613$

Van den Berghe H, Cassiman JJ, David G, Fryns JP, Michaux JL, Sokal G (1974): Distinct haematological disorder with deletion of long arm of no. 5 chromosome. Nature $\underline{251}, 437-438$

Vardiman JW, Thiele J, Arber DA, Brunning RD, Borowitz MJ, Porwit A, Harris NL, Le Beau MM, Hellstrom-Lindberg E, Tefferi A, Bloomfield CD (2009): The 2008 revision of the World Health Organization (WHO) classification of myeloid neoplasms and acute leukemia: rationale and important changes. Blood $\underline{114}, 937-951$

Wan TS, Au WY, Chan JC, Chan LC, Ma SK (1999): Trisomy 21 as the sole acquired karyotypic abnormality in acute myeloid leukemia and myelodysplastic syndrome. Leuk Res $\underline{23}, 1079-1083$

Wan TS, Ma SK, Au WY, Chan LC (2001): Derivative (1;18)(q10;q10): a recurrent and novel unbalanced translocation involving 1q in myeloid disorders. Cancer Genet Cytogenet 128, 35-38

Weisdorf DJ, Oken MM, Johnson GJ, Rydell RE (1983): Chronic myelodysplastic syndrome: short survival with or without evolution to acute leukaemia. Br J Haematol $\underline{55}$, 691-700

White AD, Hoy TG, Jacobs A (1994): Extended cytogenetic follow-up and clinical progress in patients with myelodysplastic syndromes (MDS). Leuk Lymphoma 12, 401-412

Yunis JJ, Lobell M, Arnesen MA, Oken MM, Mayer MG, Rydell RE, Brunning RD (1988): Refined chromosome study helps define prognostic subgroups in most patients with primary myelodysplastic syndrome and acute myelogenous leukaemia. Br J Haematol $\underline{68}, 189-194$

Zeidan A, Phatak P (2008): Acquired biclonal chromosome X aberrations without autosomal chromosomal anomalies in acute myeloid leukemia. Cancer Genet Cytogenet 181, 125-130

Zemanova Z, Michalova K, Buryova H, Brezinova J, Kostylkova K, Bystricka D, Novakova M, Sarova I, Izakova S, Lizcova L, et al. (2014): Involvement of deleted chromosome 5 in complex chromosomal aberrations in newly diagnosed myelodysplastic syndromes (MDS) is correlated with extremely adverse prognosis. Leuk Res $\underline{38}, 537-544$

Zini G (2017): Diagnostics and Prognostication of Myelodysplastic Syndromes. Ann Lab Med $\underline{37}$, 465-474 


\section{Danksagung}

Danken möchte ich Frau PD Dr. med. Schanz, Oberärztin der Klinik für Hämatologie und Onkologie der Universitätsmedizin Göttingen, für die Anregung zu dieser Arbeit, die anhaltende Unterstützung und die stets prägnante und konstruktive Kritik in der Betreuung, ohne die diese Arbeit wohl nicht fertiggestellt worden wäre.

Nicht versäumen zu danken möchte ich außerdem Herrn Prof. Dr. med. Haase für die Vergabe des Themas. 